University of Tennessee Health Science Center

UTHSC Digital Commons

\title{
$5-2013$
}

\section{In vitro and In vivo Characterization of the Infection Efficiency of a Modified Retrovirus Envelope Glycoprotein for Targeting Gene Transduction}

Robin L. Krueger

University of Tennessee Health Science Center

Follow this and additional works at: https://dc.uthsc.edu/dissertations

Part of the Medicine and Health Sciences Commons

\section{Recommended Citation}

Krueger, Robin L. , "In vitro and In vivo Characterization of the Infection Efficiency of a Modified Retrovirus Envelope Glycoprotein for Targeting Gene Transduction" (2013). Theses and Dissertations (ETD). Paper 129. http://dx.doi.org/10.21007/etd.cghs.2013.0166. 


\title{
In vitro and In vivo Characterization of the Infection Efficiency of a Modified Retrovirus Envelope Glycoprotein for Targeting Gene Transduction
}

\begin{abstract}
Attributes of both the viral glycoprotein and its cellular receptor play key roles in determining the outcome of infection. This body of work endeavors to illustrate how these two components influence the efficiency of virus infection in in vitro and in vivo systems with an emphasis on characterizing the transduction capacity of a novel entry-targeting glycoprotein. In previous work, the Moloney murine leukemia virus (MLV) envelope glycoprotein (Env) was modified to generate the Sst-RBS glycoprotein. This glycoprotein was created by replacing the wild type (WT) receptor binding site (RBS), located on the surface subunit (SU) of the Env, with the somatostatin peptide hormone sequence SST-14. The modifications resulted in abrogating transduction via the natural MLV receptor and redirecting transduction to a family of five somatostatin receptors (SSTR). I demonstrate that structural characteristics of the Sst-RBS glycoprotein and the intracellular fate of the SSTR receptor influence the infection efficiency of pseudotyped MLV and human immunodeficiency virus type 1 (HIV-1) based lentiviral (LV) particles. Infection and western blot assays indicate that Sst-RBS retains the structural requirements for mediating levels of transduction that are comparable to WT and approach within 5 -fold that of transduction mediated by vesicular stomatitis virus (VSV) G protein when each envelope protein is pseudotyped on MLV particles. To address the contribution of receptor characteristics on infection efficiency, HEK 293 cell lines stably expressing comparable cell surface levels of SSTR-2, SSTR-3 and SSTR-5; which have natural differences in intracellular trafficking; were generated. Infection assays revealed that distinctive SSTR subtype-specific destinations correlated with observable differences in the level of Sst-RBS MLV and LV transduction. Taken together the results of virus binding, internalization kinetics, $\mathrm{pH}$-neutralizing agents, protease inhibitor and penetration assays support that SSTR- 5 allows a greater level of transduction because viruses internalized by this subtype are exposed to more permissive intracellular compartments. Specifically, SSTR-5-associated virions are directed to compartments that are more favorable to cytosolic penetration of viral cores than the compartment(s) to which virions bound to subtypes 2 and 3 are directed; possibly due to a more beneficial complement of host cell proteases. These data suggested that receptor characteristics such as the intracellular fate of internalized virus-receptor complexes exert a strong influence on the efficiency of infection. Surprisingly, even though the difference in the in vitro transduction capacity of Sst-RBS and VSV G pseudotyped LV particles was greater than that of the MLV pseudotypes, the difference did not translate to a reduction in in vivo transduction capacity. A pilot study examining the feasibility of in vivo transduction demonstrated proof of principle and identified regions of the murine brain with endogenous surface expression of SSTRs that were as efficiently transduced by SstRBS LV as by VSV G LV.
\end{abstract}

\section{Document Type \\ Dissertation}

\section{Degree Name}

Doctor of Philosophy (PhD)

\section{Program}

Biomedical Sciences

Research Advisor

Lorraine M. Albritton, Ph.D. 


\section{Keywords}

Entry, Glycoprotein, Retrovirus, Targeting

\section{Subject Categories}

Medicine and Health Sciences 
In Vitro and In Vivo Characterization of the Infection Efficiency of a Modified Retrovirus Envelope Glycoprotein for Targeting Gene Transduction

\author{
A Dissertation \\ Presented for \\ The Graduate Studies Council \\ The University of Tennessee \\ Health Science Center
}

In Partial Fulfillment

Of the Requirements for the Degree

Doctor of Philosophy

From The University of Tennessee

By

Robin L. Krueger

May 2013 
Portions of Chapters 1, 3 and 5 (C) 2012 by American Society for Microbiology. Portions of Chapter 4 (C) 2013 by American Society for Microbiology. All other material @ 2013 by Robin L. Krueger.

All rights reserved. 


\section{Dedication}

This is dedicated to my husband Aaron Krueger and my parents Paul and Kathy Stoner for their enduring support and encouragement. LUATT and thank you. 


\section{Acknowledgements}

I would like thank my graduate advisor, Lorraine Albritton, PhD and my advisory committee: Jonathon McCullers, MD; Stacey Schultz-Cherry, PhD; Tiffany Seagroves, PhD and Michael Whitt, PhD for their helpful comments and support. I would also like to acknowledge previous lab members Fang Li, PhD and Byoung Ryu, PhD for their previous work on the Sst-RBS glycoprotein and the manuscript Li, F., Ryu, B.Y., Krueger, R.L., Heldt, S.A., and Albritton, L.M., Targeted entry via somatostatin receptors using a novel modified retrovirus glycoprotein that delivers genes at levels comparable to those of wild-type viral glycoproteins. J Virol, 2012. 86(1): p. 373-81. I earned an equal second authorship on this manuscript for my contributions of the data presented in Figures 6 and 7, assistance with lentivirus titer data interpretation and with revisions and response to reviewers. I also thank Ramya Balasubramaniam for her efforts in sorting the SSTR expressing cell lines, and Krish Kizhatil, PhD and Tatiana Zavorotinskaya, $\mathrm{PhD}$ for methods. Additionally, I want to recognize all the faculty and staff of the University of Tennessee Health Science Center Department of Microbiology, Immunology and Biochemistry for providing a friendly environment for learning and research; in particular Xiaofei Wang for tissue sectioning and immunostaining assistance, John Cox, PhD for microscopy assistance, and Tim Higgins who generated digital photographs of infection assays for quantification purposes. Finally, Scott $A$. Heldt, PhD for performing microinjections of pseudotyped lentivirus in mice and Ashley Ezekiel for generating Aperio ScanScope images.

This research was supported by National Institutes of Health grant R01CA081171 from the National Cancer Institute to L.M.A. and bridge funding provided by the University of Tennessee Health Science Center, College of Health Sciences. 


\begin{abstract}
Attributes of both the viral glycoprotein and its cellular receptor play key roles in determining the outcome of infection. This body of work endeavors to illustrate how these two components influence the efficiency of virus infection in in vitro and in vivo systems with an emphasis on characterizing the transduction capacity of a novel entry-targeting glycoprotein. In previous work, the Moloney murine leukemia virus (MLV) envelope glycoprotein (Env) was modified to generate the Sst-RBS glycoprotein. This glycoprotein was created by replacing the wild type (WT) receptor binding site (RBS), located on the surface subunit (SU) of the Env, with the somatostatin peptide hormone sequence SST-14. The modifications resulted in abrogating transduction via the natural MLV receptor and redirecting transduction to a family of five somatostatin receptors (SSTR). I demonstrate that structural characteristics of the Sst-RBS glycoprotein and the intracellular fate of the SSTR receptor influence the infection efficiency of pseudotyped MLV and human immunodeficiency virus type 1 (HIV-1) based lentiviral (LV) particles. Infection and western blot assays indicate that Sst-RBS retains the structural requirements for mediating levels of transduction that are comparable to WT and approach within 5-fold that of transduction mediated by vesicular stomatitis virus (VSV) G protein when each envelope protein is pseudotyped on MLV particles. To address the contribution of receptor characteristics on infection efficiency, HEK 293 cell lines stably expressing comparable cell surface levels of SSTR-2, SSTR-3 and SSTR-5; which have natural differences in intracellular trafficking; were generated. Infection assays revealed that distinctive SSTR subtype-specific destinations correlated with observable differences in the level of Sst-RBS MLV and LV transduction. Taken together the results of virus binding, internalization kinetics, $\mathrm{pH}$-neutralizing agents, protease inhibitor and penetration assays support that SSTR-5 allows a greater level of transduction because viruses internalized by this subtype are exposed to more permissive intracellular compartments. Specifically, SSTR-5-associated virions are directed to compartments that are more favorable to cytosolic penetration of viral cores than the compartment(s) to which virions bound to subtypes 2 and 3 are directed; possibly due to a more beneficial complement of host cell proteases. These data suggested that receptor characteristics such as the intracellular fate of internalized virusreceptor complexes exert a strong influence on the efficiency of infection. Surprisingly, even though the difference in the in vitro transduction capacity of Sst-RBS and VSV G pseudotyped LV particles was greater than that of the MLV pseudotypes, the difference did not translate to a reduction in in vivo transduction capacity. A pilot study examining the feasibility of in vivo transduction demonstrated proof of principle and identified regions of the murine brain with endogenous surface expression of SSTRs that were as efficiently transduced by Sst-RBS LV as by VSV G LV.
\end{abstract}




\section{Table of Contents}

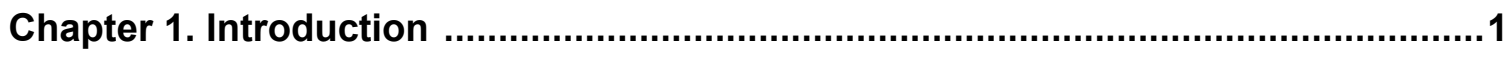

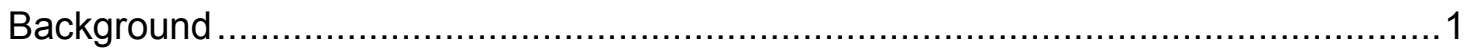

Moloney Murine Leukemia Virus ....................................................................

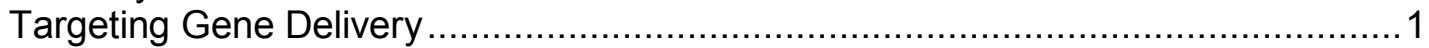

Sst-RBS: A Modified MLV Envelope Glycoprotein ........................................ 2

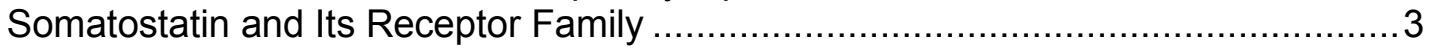

Intracellular Trafficking of Somatostatin Receptors ......................................... 4

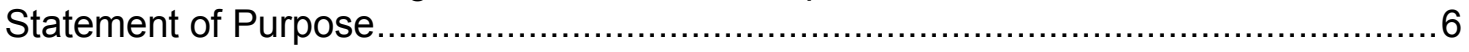

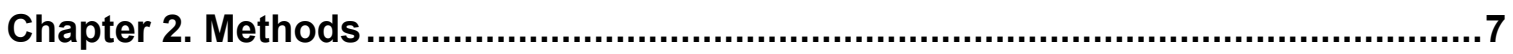

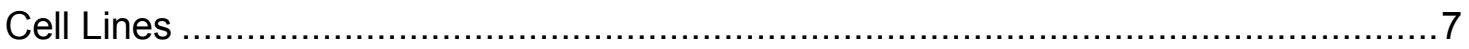

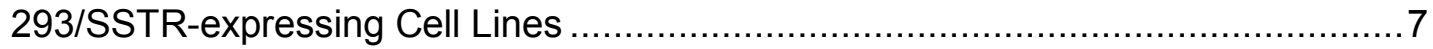

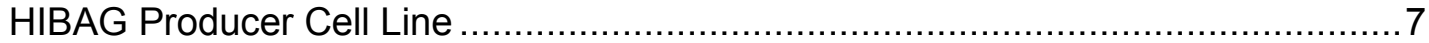

Plasmid Construction ..................................................................................... 7

Sst-RBS and Flexible Flanking Sequence Modified Glycoproteins....................... 7

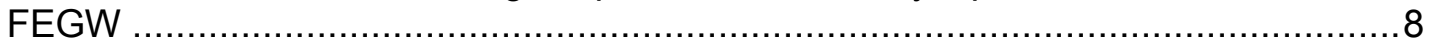

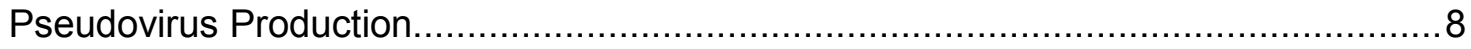

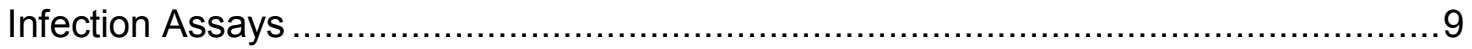

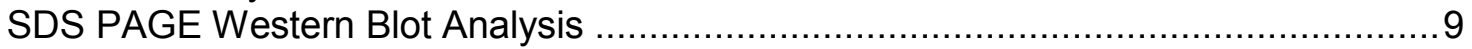

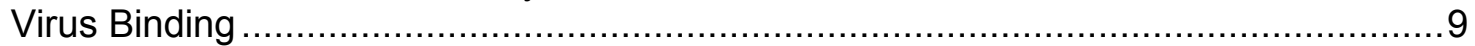

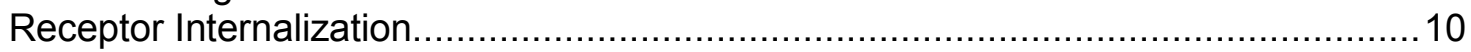

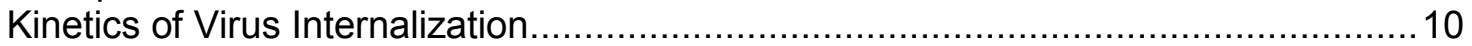

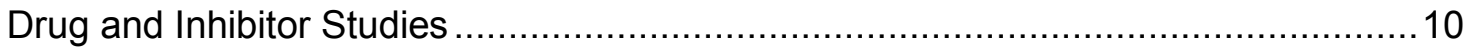

Quantitative Real-time PCR Analysis .......................................................... 11

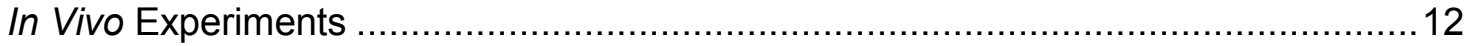

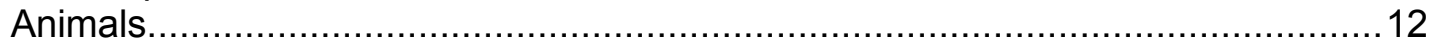

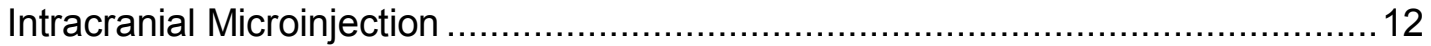

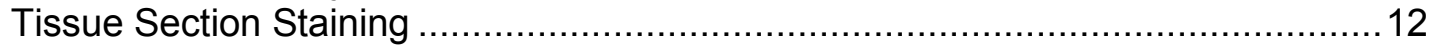

Transduction Zone Measurements ............................................................. 13

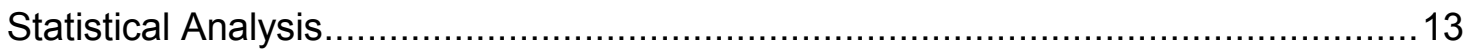

\section{Chapter 3. Targeted Entry via Somatostatin Receptors Using a Novel Modified Retrovirus Glycoprotein that Delivers Genes at Levels Comparable to Those of Wild-Type Viral Glycoproteins.......................................................................... 14}

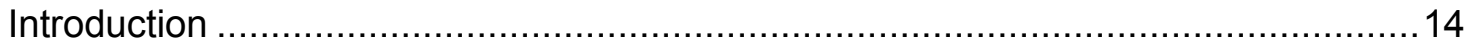

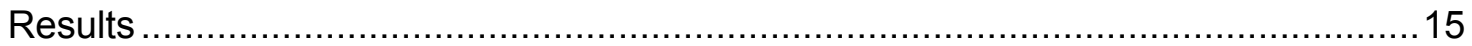

Sst-RBS Is Capable of Transducing 293/SSTR-5 Cells at Levels Comparable to

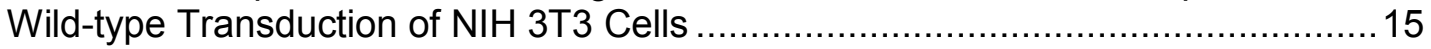

Pseudovirion Binding to SSTR-5 Is Comparable to mCAT-1........................... 15

A Non-flexible Turn Flanking the SST-14 Ligand Insertion Is Required for

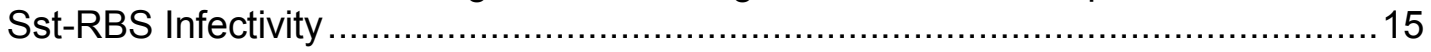

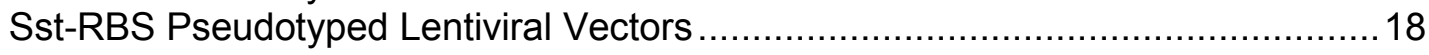

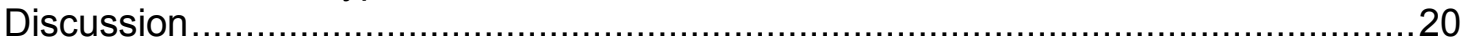


Chapter 4. Characteristics of the Cellular Receptor Influence the Intracellular

Fate and Efficiency of Retrovirus Infection ..................................................... 23

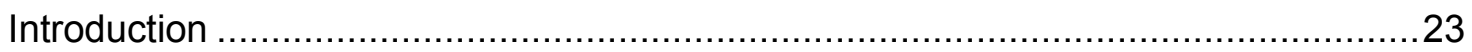

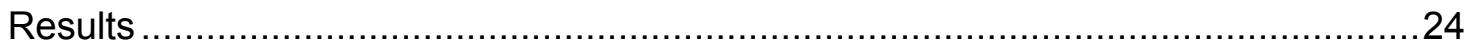

Infection of Sst-RBS Pseudotyped Viruses Is Greatest via SSTR-5 ....................24

Receptor Specific Interactions Mediate Sst-RBS MLV Infection..........................27

The Relative Level of Cell-associated Virus Can Not Account for Differences in

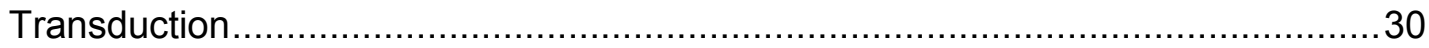

Infection of Sst-RBS Pseudovirions Requires Internalization ............................. 33

A Slower Rate of Internalization Correlates with Enhanced Infection .....................33

SSTR-5 Directs Virus to a More Permissive Environment................................... 37

Penetration into the Host Cytoplasm Is More Efficient via SSTR-5 ..................... 41

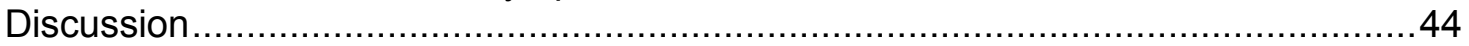

\section{Chapter 5. In Vivo Gene Transduction with Sst-RBS Pseudotyped Lentivirus....... 49}

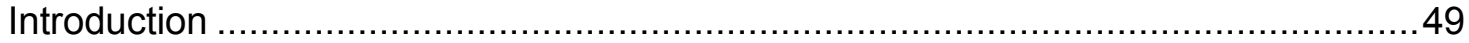

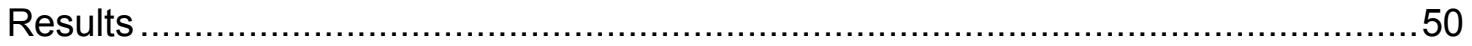

Proof of Principle for In Vivo Gene Transduction by Sst-RBS Pseudotyped

Lentivirus Particles ...................................................................................... 50

SSTR-2 Expression in the Murine Brain..................................................... 50

Sst-RBS Lentivirus Mediated Transduction in the Murine Brain ..........................54

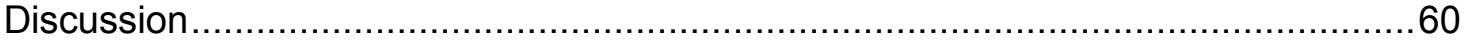

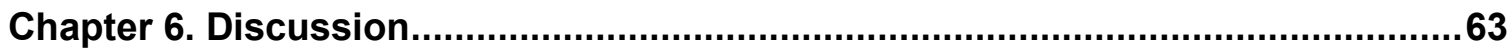

Identify the Endocytic Mechanisms and Intracellular Destination of Sst-RBS Entry ...63

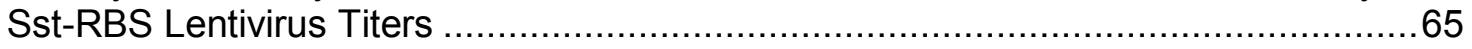

Targets for Sst-RBS-Mediated Gene Delivery In Vivo .........................................67

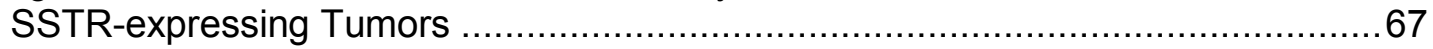

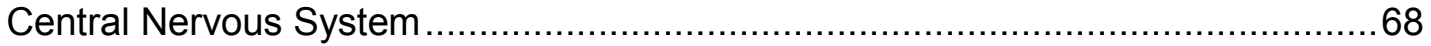

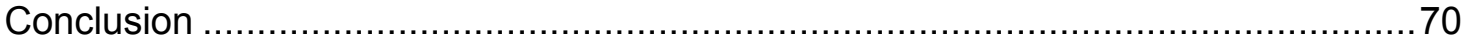

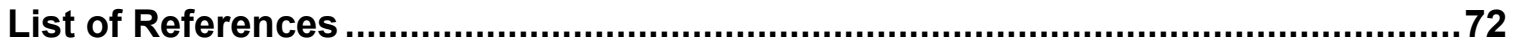

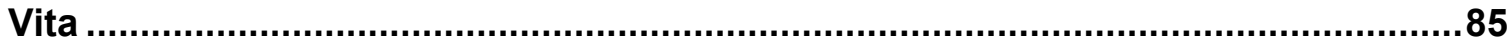




\section{List of Figures}

Figure 1-1. Schematic of the Sst-RBS and wild-type glycoprotein ......................... 3

Figure 1-2. Somatostatin receptor subtypes have different intracellular fates ..............5

Figure 3-1. Sst-RBS glycoprotein targets entry at levels comparable to those of entry mediated by wild-type Moloney murine leukemia virus glycoprotein and approaching those of VSV G pseudovirions

Figure 3-2. Generation of a host cell line stably expressing levels of target receptor SSTR comparable to the levels of ecotropic receptor on NIH 3T3 cells .... 17

Figure 3-3. Modified Sst-RBS glycoproteins containing the SST-14 ligand..... 18

Figure 3-4. Glycoproteins with a non-flexible peptide linker sequence upstream of the SST-14 ligand retain infectivity

Figure 3-5. Lentivirus transducing an EF1 $\alpha$-GFP transgene exhibits higher expression

Figure 4-1. SSTR-expressing cell populations display comparable levels of target receptors on their cell surface

Figure 4-2. Infection of pseudotyped viruses is greatest via SSTR-5

Figure 4-3. Receptor-specific interactions mediate Sst-RBS MLV infection 28

Figure 4-4. The SST-14 ligand does not act by inhibiting cell division 29

Figure 4-5. The relative level of cell-associated virus is comparable for each SSTRexpressing cell line.

Figure 4-6. Infection of Sst-RBS pseudovirions requires internalization. 34

Figure 4-7. Patterns of receptor internalization among SSTR subtypes. 35

Figure 4-8. A slower rate of virion internalization correlates with increased infection...38

Figure 4-9. Neutralizing acidic compartments enhances infection via SSTR-2 39

Figure 4-10. SSTR-5 internalizes to a more permissive intracellular environment..... 42

Figure 4-11. Virus internalized via SSTR-5 more efficiently penetrates into the host cytoplasm.

Figure 4-12. Model of the influence of intracellular destination on infection 45

Figure 5-1. Proof of principle for in vivo gene transduction by Sst-RBS pseudotyped lentivirus. 
Figure 5-2. SSTR-2 receptors are expressed in the murine brain ..........................52

Figure 5-3. SSTR-2 receptors are expressed on neurons of the murine brain ............53

Figure 5-4. SSTR-2 receptors are expressed on murine ependymal cells .................55

Figure 5-5. Sst-RBS lentivirus mediated transduction of murine ependymal cells .......56

Figure 5-6. Sst-RBS and VSV G lentivirus mediated transduction along the needletracks in the striatum of mice ........................................................ 57

Figure 5-7. Transduction zone diffusion of Sst-RBS and VSV G lentivirus particles in intracranially injected mice..................................................... 58 


\section{List of Abbreviations}

AAV .............. Adeno associated virus (followed by number indicates subtype)

AD ................ Alzheimer's disease

ADV .............. Adenovirus

ALS ............... Amyotrophic lateral sclerosis

APOBEC3 ..... Apolipoprotein B mRNA-editing enzyme catalytic polypeptide-like 3 family

ASLV-A ......... Avian sarcoma leukemia virus subtype A

BafA ............. Bafilomycin A1

BBB.............. Blood brain barrier

CA............... Capsid protein

CA074 Me ..... Cathepsin B inhibitor

CC................ Cerebral cortex

CCR5 ........... HIV-1 co-receptor (C-C chemokine receptor type 5; CD195)

CD4.............. HIV-1 primary receptor (cell surface cluster of differentiation 4)

Chlor ............ Chloroquine

ChP .............. Choroid plexus

CNS ............. Central nervous system

$\mathrm{CP}$............... Caudate putamen of the corpus striatum

CSF.............. Cerebral spinal fluid

CTD ............. C-terminal domain of MLV SU

CXCR4 .......... HIV-1 co-receptor; SDF-1 receptor (C-X-C chemokine receptor 4)

DG .............. Dentate gyrus of the hippocampus

DMSO ........... Dimethyl sulfoxide

DN............... Dominant negative

EE ................ Early endosome

ENTV ............ Enzootic nasal tumor virus

Env............... Envelope glycoprotein

ErbB ............. Epidermal growth factor receptor

ERT ............. Enzyme replacement therapy

EtOH ............ Ethanol

FACS ........... Fluorescence activated cell sorting

FEGW .......... SIN HIV-1 based GFP expression genome under control of hEF1 $\alpha$

FUGW .......... SIN HIV-1 based GFP expression genome under control of hUbC 
Fv1 .............. Friend virus susceptibility factor-1

GFAP ........... Glial fibrillary acidic protein

GFP ............ Green fluorescent protein

GPCR …....... G-protein coupled receptor

gRNA ........... Genomic RNA

GUSB............ $\beta$-glucuronidase enzyme

HA ............... Influenza hemagglutinin epitope (YPYDVPDYA) tag

HD................ Huntington's disease

hEF1 $\alpha$........... Human Elongation Factor $1 \alpha$ promoter

HIV-1 ............ Human immunodeficiency virus type 1

hpi ................ Hours post infection

hUbC............ Human ubiquitin $\mathrm{C}$ promoter

LacZ .............. $\beta$-galactosidase enzyme

LE ................ Late endosome

LSD .............. Lysosomal storage disease

LtV ............... Lateral Ventricle

LV ................ Lentivirus

M $\beta C D$............ Methyl- $\beta$-cyclodextrin

mCAT-1 ......... MLV receptor (mouse cationic amino acid transporter)

MFI............... Mean fluorescent intensity

mKate2 .......... Far-red fluorescent protein

MLV ............. Murine leukemia virus

MPS ............. Mucopolysaccharidosis type VII

MSS ............. Minus strand strong stop signal

NET .............. Neuroendocrine tumor

NeuN............ Neuronal nuclear membrane protein

NIH 3T3........ Murine fibroblast cells

NSC ............. Neuronal stem cell

NTF .............. Neurotrophic factor

p24 ELISA.... HIV-1 capsid protein (p24) enzyme-linked immuno sorbent assay

PBS............. Phosphate buffered saline ( $\mathrm{pH} 7.2)$

PD ................ Parkinson's disease

PIC .............. Pre-integration complex

PRR ............. Proline rich region of MLV SU 
Rab5 ........... Rab GTPase 5, early endosomal

Rab7 ........... Rab GTPase 7, late endosomal

Rab11 .......... Rab GTPase 11, recycling endosomal

RBD ............ Receptor binding domain of MLV SU

RBS ............. MLV receptor binding site

RE ............... Recycling endosome

RMS ............. Rostral migratory stream

RNAi ............ RNA interference

RV ............... Retrovirus

SDS PAGE.... Sodium dodecyl sulfate polyacrylamide gel electrophoresis

SGZ ............. Subgranular zone

shRNA.......... Short hairpin RNA

SIN ............... Self-inactivating lentiviral vector

SiRNA ........... Small interfering RNA

SN................ Subthalamic nucleus

SSA.............. Somatostatin analog

SSTR ........... Somatostatin receptor (followed by number indicates subtype)

SST-14......... Somatostatin 14 peptide ligand

SST-28......... Somatostatin 28 peptide ligand

Sst-RBS ....... MLV glycoprotein in which the WT RBS is replaced with SST-14

SU................ Surface subunit of the MLV Env

SVZ.............. Subventricular zone

TGN ............. Trans-golgi network

TM................ Transmembrane subunit of the MLV Env

Trim5 $\alpha$......... Tripartite motif-containing protein $5 \alpha$ isoform

$\mathrm{TU} / \mathrm{ml}$............ Transducing units per milliliter

TZ ................ Transduction zone

$\mathrm{T}_{50} \ldots \ldots \ldots \ldots . . . . .$. Time to reach $50 \%$ of transduction

Vif................ Viral infectivity factor; HIV-1 accessory protein

Vpu .............. Viral protein U; HIV-1 accessory protein

VSV G.......... Vesicular stomatitis virus $G$ protein

WT ............... Wild type

X-Gal............ LacZ substrate (5-bromo-4-chloro-indolyl-b-D-galactopyranoside)

293 ............... Human embryonic kidney (HEK) 293 cells

293/SSTR ..... 293 cells stably expressing the indicated SSTR subtype 


\section{Chapter 1. Introduction *}

\section{Background}

\section{Moloney Murine Leukemia Virus}

Moloney murine leukemia virus (MLV), a member of the genus gammaretrovirus in the family Retroviridae; is an enveloped virus containing two copies of a positive-sense, single-stranded RNA genome. An ecotropic retrovirus (RV), Moloney MLV initiates infection by binding its host cell receptor mCAT-1 (a mouse cationic amino acid transporter) to gain entry into host murine cells [1-3]. This interaction is mediated by the surface subunit (SU) of the MLV envelope protein (Env) which contains the receptor binding site (RBS). The internalization of MLV-mCAT-1 complexes has been to shown to occur independently from classical clathrin-mediated endocytosis by a caveolinassociated pathway [4-6]. Following endocytic events, host cell cathepsin proteases found in the late endosomal compartment cleave the viral Env [7] and trigger conformational changes in both the SU and transmembrane (TM) domains of Env that allow the TM fusion peptide to mediate fusion between the virus and host cell membranes [8]. After the virion core is uncoated and released into the host cytosol a pre-integration complex (PIC) is formed which includes (among other components) a double stranded DNA copy of the viral RNA, which has been reverse transcribed by the viral reverse transcriptase enzyme, and the viral integrase enzyme. The PIC is transported into the host cell nucleus while the nuclear membrane is dissolved during mitotic events $[9,10]$ and the integrated DNA, or provirus, becomes a template for the generation of new infectious virions by host cell machinery. The entry events of lentiviruses (LV) such as human immunodeficiency virus type 1 (HIV-1) involve many of the same requirements as MLV with a notable exception that nuclear import of the PIC does not rely on host cell mitosis, but rather gains access through nuclear pore complexes which allow PIC transport across the intact nuclear membrane. It is generally thought that whereas MLV integration occurs only in actively dividing cells, HIV-1 is capable of integration in both dividing and non-dividing cells [10]. Consequently, LV is considered a more appropriate vector for in vivo gene delivery where a majority of target cells are quiescent (i.e, not actively dividing).

\section{Targeting Gene Delivery}

Since the provirus is integrated into the host cell's chromosome, RVs and LVs are attractive solutions for investigators interested in targeting delivery of therapeutic genes to the cell and tissue types most likely to benefit from such therapies. To this end, numerous attempts at re-directing the MLV Env to new target cells have been published (for reviews see $[11,12]$ ). The most frequently used insertion sites include the amino-terminus and the proline-rich region (PRR) of the Moloney MLV SU glycoprotein

\footnotetext{
*Adapted with permission. Li, F., B. Y. Ryu, R. L. Krueger, S. A. Heldt and L. M. Albritton. Targeted entry via somatostatin receptors using a novel modified retrovirus glycoprotein that delivers genes at levels comparable to those of wild-type viral glycoproteins. J Virol, 2012. 86(1): 373-381.
} 
but to date these strategies have remained largely unsuccessful [11-13]. Although the chimeric virions were generally able to bind their receptors and internalize, membrane fusion was impaired resulting in little to no transduction in target cells lacking the natural virus receptor [13-15]. These impairments are more pronounced when ligands are inserted in the amino-terminus than in the PRR of the SU [14]. Additionally, altering critical residues in the amino-terminus blocks infection at a hemi-fusion point [8]. While simple RVs, such as MLV, have found specific uses as vectors for delivering genes in cell culture and ex vivo therapies (removing cells from the body and transducing them before re-implantation) targeting hematopoietic stem cells [13] they have limited use in vivo, however. This is mainly due to the restrictions imposed on them from cell division requirements during provirus integration and a capacity to induce oncogenesis through unregulated integration events [16]. Strategies utilizing other virus species have been developed and involve antibody-based strategies using single-chain fragment variable (scFv) fused to the c-terminus of the measles virus hemagglutinin $\mathrm{H}$ protein [17-19] or the immunoglobulin Fc-binding ZZ domain from Staphylococcus aureus protein A into the E1 glycoprotein from Sindbis virus [20]. The in vivo potential for these systems is also limited because low levels of viral membrane incorporated glycoproteins occurred due to aggregation of the scFv leading to incorrect assembly [21] as well as unstable interactions [20] and rapid vector inactivation by compliment [22]. Lentiviral based strategies might be more effective for in vivo targeting efforts because they are capable of transducing terminally differentiated cells which may no longer be actively dividing. Lentivirus vectors pseudotyped with the glycoprotein from amphotropic MLV [23] and the vesicular stomatitis virus (VSV) G protein [24], both of which are capable of infecting nearly every mammalian cell type, have been widely used for gene delivery [13]. However, the broad host ranges of these two proteins do not reflect true targeted delivery to specific cell types.

\section{Sst-RBS: A Modified MLV Envelope Glycoprotein}

A new targeting strategy was devised by previous researchers in this laboratory based on structural similarities of the Moloney MLV RBS and a small peptide hormone, somatostatin 14 (SST-14) [25]. The resulting modified Env glycoprotein, Sst-RBS, was created by replacing the wild type (WT) RBS (residues C73 to C86 of Moloney MLV) with the SST-14 sequence and short amino- and carboxy-peptide linker sequences (Figure 1-1) [25]. The linker sequences were designed to form rigid structural turns directing the SST-14 sequence up and away from the SU, in a manner similar to the published structure of the Friend strain of MLV [26]. The modifications that created SstRBS resulted in a switch from the use of mCAT-1 to somatostatin receptors (SSTR) [25], a family of multi-membrane spanning $G$ protein-coupled receptors (GPCR). These receptors are not entry receptors for any known virus and ordinarily recognize SST-14, related ligands and peptide mimetics. Replacing the WT RBS with a ligand that binds a human transmembrane receptor has several advantages. First, it has the greatest chance of maintaining the virus's natural mechanism of entry, except of course, that infection would occur via the new receptor. Second, it reduces issues related to structure that affect protein folding and virion assembly. Lastly, it abolishes the natural murine host specificity of the virus generating clear evidence that the virus has been retargeted. 


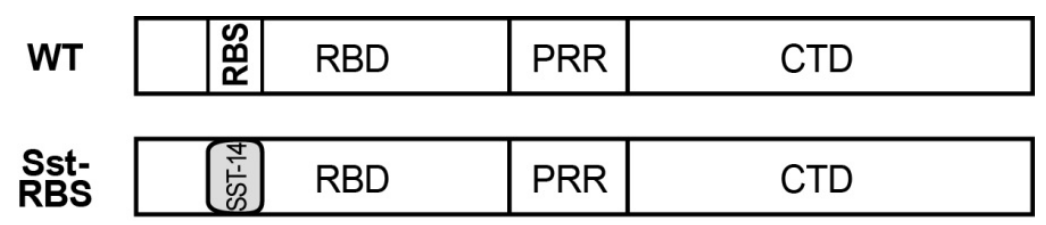

Figure 1-1. Schematic of the Sst-RBS and wild-type glycoprotein

Schematic representation of the surface subunit of wild type ecotropic Moloney (WT, Top) and Sst-RBS (Bottom) envelope glycoproteins with the locations of the receptor binding site (RBS) in WT and the peptide sequence YASAGCKNFFWKTFTSCAYTAS containing the SST-14 ligand (underlined) flanked by short peptide linkers. RBD: Receptor Binding Domain, PRR: Proline Rich Region, CTD: Carboxy-Terminal Domain.

\section{Somatostatin and Its Receptor Family}

SST-14 is a small peptide hormone that primarily exerts regulatory effects on cells of the endocrine and exocrine systems in mammals and fish. Notably, it inhibits the release of other hormones such as growth hormone and somatotropin, functions as a neurotransmitter, and has anti-proliferative and cell differentiation actions. Two biologically active forms are produced by preproprotein cleavage: a 14 (SST-14) and a 28 (SST-28) amino acid peptide. The SST-14 cleavage product is most commonly used as a receptor agonist; both ligands form a hairpin loop structure stabilized by an internal disulfide bond [27]. The sequence FWKT, located at the center of the hairpin, is required for functional binding to a family of five multi-membrane spanning GPCRs [28]. The five SSTR subtypes: SSTR-1, SSTR-2 (A and B), SSTR-3, SSTR-4, and SSTR-5 can be classified into two groups based on structural and pharmacological properties. Group 1 includes SSTR-2 (A and B), SSTR-3 and SSTR-5 whereas SSTR-1 and SSTR-4 belong to Group 2. Group 1, but not Group 2, receptors are capable of binding important SST analogues such as octreotide [29] and are more efficiently internalized after agonist exposure [30]. Subsets of SSTR monomers, homo- and heterodimers are naturally expressed in many cell types; however, the SSTR-2A isoform is the most widely distributed [29]. Additionally, SSTRs may heterodimerize with other receptors such a dopamine type 2 [31]; epidermal growth factor receptor, ErbB [32] and the mu-opioid receptor, MOR1 [33]. Surface expression of SSTRs is abundant in the brain; gastrointestinal tract; exo- and endocrine pancreas and pituitary and have been identified in multiple tumor types and cell lines [27, 28].

Despite the gene for each SSTR subtype being located on separate chromosomes, the SSTRs display remarkable sequence homology. There is $39-57 \%$ homology between the five subtypes with most variability occurring in the carboxy-terminal cytoplasmic tails and a notable conserved motif located in transmembrane domain VII. The SSTR also show 82-99\% homology across mammalian species [27]. As would be expected based on such similar homology, SSTR subtypes bind SST-14 with comparable nanomolar affinities [27, 29]; however, binding affinities between the SSTRs and other ligands do vary. For instance, while SSTR-2 and SSTR-3 bind SST-28 with similar affinities to SST-14, SSTR-5 has a higher affinity for SST-28 [27, 30]. Furthermore, octreotide, a synthetic octapeptide that mimics the agonist effects of the natural SST ligand, binds SSTR-2 with a higher affinity than both SSTR-3 and SSTR-5 
$[27,34]$. In addition to the range of ligand-receptor affinities there is also evidence that various ligands may change the degree of signaling or endocytosis based on different conformations of the ligand-receptor complex [34]. As an example, the non-peptide ligand L-779,976 binds to SSTR-2 with the same affinity as SST-14, but is 10 times more potent at inhibiting cAMP production (a common measure of SSTR signaling) and yet could only produce $70 \%$ of SST-induced receptor internalization [35]. These differences might be attributable to a relatively large,[27] putative ligand binding pocket which is thought to span multiple residues across transmembrane domains III-VII [29].

\section{Intracellular Trafficking of Somatostatin Receptors}

A common feature of GPCRs is agonist induced receptor desensitization. Agonist binding stimulates GPCR signaling which it is regulated, in part, by phosphorylation of intracellular receptor domains. Receptor phosphorylation also recruits $\beta$-arrestins. Depending on the class of GPCR, $\beta$-arrestin may not be stably associated with the desensitized receptor complex, thus while the desensitization process generally (but not always) involves receptor internalization, $\beta$-arrestin might not be internalized with the complex. Internalization of the GPCR is followed by receptor degradation or resensitization/recycling. These actions allow the cells to correspondingly either down-regulate or preserve the amount of unbound receptors on the cell surface during prolonged agonist exposure. In some cases, mobilization of intracellular stores quickly up-regulates surface receptors. The pattern of phosphorylation and $\beta$-arrestin recruitment determines the extent of SSTR internalization, resensitization and recycling; and depends, to a large degree, on the presence and location of clusters of Ser and Thr phosphate acceptor sites [34]. Because the carboxy-terminal cytoplasmic tails differ among SSTR subtypes 2, 3 and 5 with respect to the amount and position of putative phosphorylation sites and $\beta$-arrestin recruitment, so does their method of desensitization $[30,36]$. As a result, the intracellular fates of the ligand-receptor complexes are also distinct (Figure 1-2).

In the absence of agonist, SSTR-2 and SSTR-3 are almost entirely localized at the plasma membrane, but a large amount of SSTR-5 can be found in intracellular pools in certain cell types [34, 37]. After agonist exposure, phosphorylation of clusters of acceptor sites occurs in SSTR-2 and SSTR-3, but these clusters are absent in the cytoplasmic tail of SSTR-5. Stable $\beta$-arrestin complexes are only associated with SSTR-2 and their formation is dependent on G protein-receptor kinase (GRK)-mediated phosphorylation. Conversely, $\beta$-arrestin is only transiently associated with SSTR-3 and SSTR-5. SSTR-2 and SSTR-3 are rapidly internalized in response to $\beta$-arrestin recruitment, and at early time points both are co-localized with transferrin; a commonly used early endosome marker [36]. SSTR-2 is recycled back to the plasma membrane through the trans-golgi network (TGN) [30] without any detectable loss of the receptor [36]. Various SST analogues are reported to have differing fates when internalized with SSTR-2: SST-14 is degraded by cellular peptidases located in the early endosome and the cleavage products are subsequently released. Endothelin-converting enzyme-1 (ECE-1) has been implicated; although treatment with the ECE-1 inhibitor SM-19712 only partially inhibits SST-14 degradation; indicating other proteases may also be responsible. Octreotide, however, is released unaltered from cells [38]. After SST-14 induced endocytosis of SSTR-3, only a small amount of the receptor is redistributed back to the plasma membrane. Most SSTR-3 loses transferrin association as the 


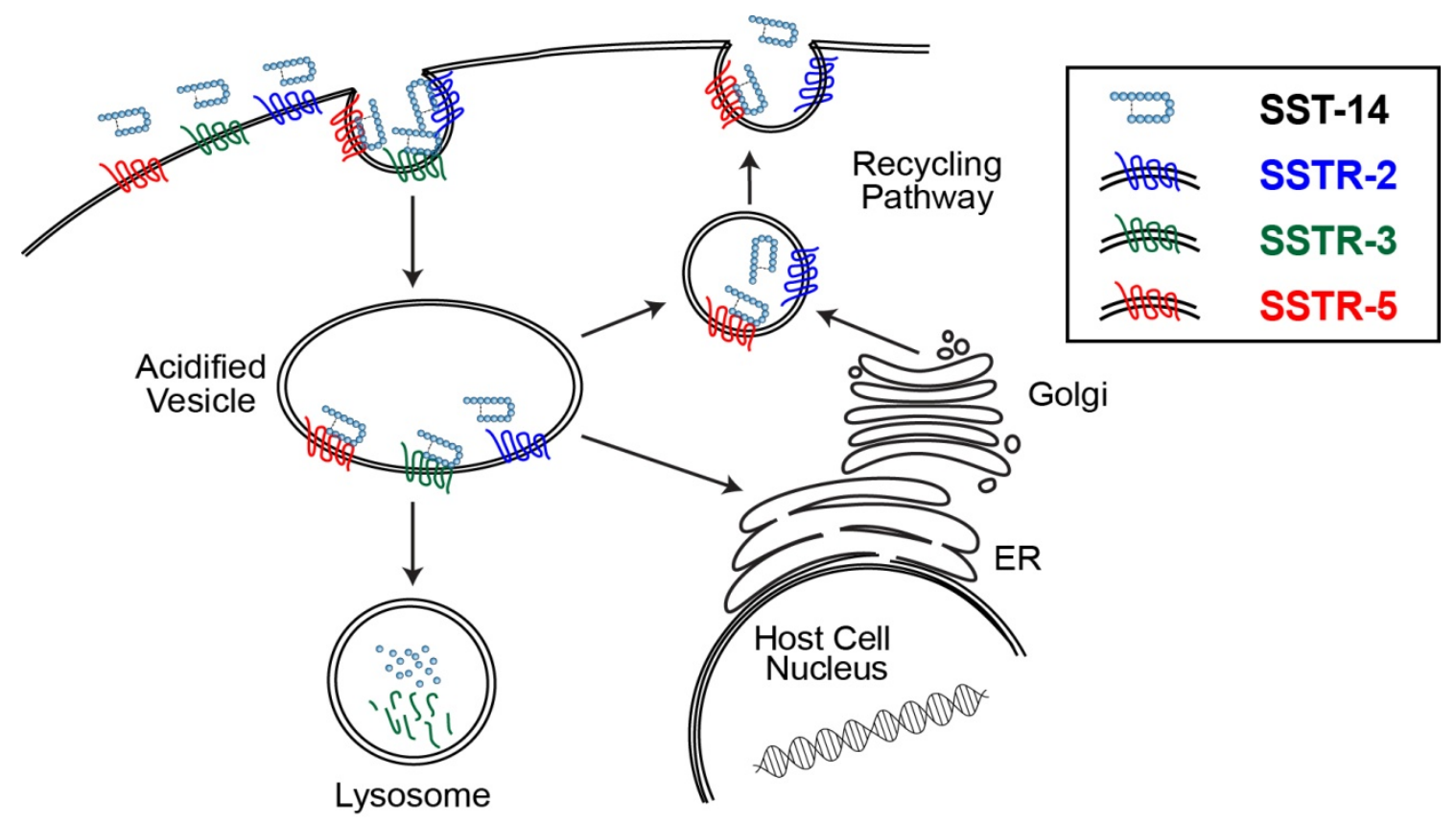

Figure 1-2. Somatostatin receptor subtypes have different intracellular fates

Following SST-14 ligand-induced receptor internalization, each SSTR subtype is trafficked to acidified vesicles. During the process of desensitization, the ligand is proteolytically cleaved from SSTR-2 (blue), the receptor is returned to the plasma membrane and free ligand is released through the recycling pathway. SST-14 bound to SSTR-3 (green) is directed to the lysosomal compartment and both ligand and receptor are degraded. The intracellular fate of SSTR-5 (red) is unclear, but it may also be recycled back to the cell surface. 
process progresses, undergoes ubiquitin-dependent lysosomal degradation and is consequently down-regulated in response to prolonged SST-14 exposure [36]. In rat insulinoma (RIN) cells expressing SSTR-3, SST-14 colocalizes with the lysosomal protease cathepsin $\mathrm{D}$, is degraded in a low $\mathrm{pH}$ dependent manner and products are released from the cell. In contrast, octreotide is released back into the supernatant intact and SSTR-3 is continuously recycled [39]. When SST-14 is internalized with SSTR-5 in COS-7 cells, the level of surface SSTR-5 is quickly replenished through a combination of mobilization from intracellular pools and receptor recycling while internalized ligand is directed to an unidentified perinuclear compartment, possibly lysosomes [37]. From the wide range of literature on the subject, it is clear that species, ligand and cell-type specific intracellular events make desensitization an extremely dynamic process.

\section{Statement of Purpose}

Several approaches to modifying viral glycoproteins to address the idea of targeting gene delivery have been developed (reviewed in [11-13]). To date, the main approach has been to insert ligands and proteins into various regions of virus Env proteins in order to redirect the virus to new host cell receptors. This strategy has been ineffective because these chimeras remain incapable of triggering the correct conformation changes in Env that allow membrane fusion. Creating effective therapeutic viral vectors entails generating a virus with the greatest potential for transduction and it is clear that the largest barrier to this requirement has been membrane fusion. Attributes of both the viral glycoprotein and its cellular receptor play key roles in mediating events such as membrane fusion capacity and influence the efficiency of infection. The modifications employed during development of the Sst-RBS glycoprotein retain the potential for membrane fusion; however, the question remains whether Sst-RBS is useful for targeting gene delivery in vivo. The goal of this dissertation is to characterize the infection efficiency of the Sst-RBS glycoprotein and demonstrate its potential for targeting in vivo gene delivery.

- The objective of Chapter 3 is to demonstrate that characteristics of the Sst-RBS glycoprotein influence infection efficiency by evaluating the structural requirements of the SST-14 replacement region. An additional goal is to determine the capacity for in vitro transduction by Sst-RBS compared to WT and VSV G envelope proteins in an effort to develop a high capacity LV vector suitable for targeting in vivo gene delivery.

- The objective of Chapter 4 is to identify characteristics of the cellular receptor that influence infection. By investigating how intracellular events following receptor endocytosis affect the outcome of infection I hope to assess if specifically targeting receptors with certain intracellular pathways should be considered in the development of future targeting vectors.

- The objective of Chapter 5 is to demonstrate proof of principle for in vivo transduction and identify regions of the murine brain with endogenous surface expression of SSTRs that are susceptible to infection by the Sst-RBS glycoprotein. 


\section{Chapter 2. Methods}

\section{Cell Lines}

Cells were maintained in Dulbecco's Modified Eagle Medium (DMEM) high glucose supplemented with $8 \%$ donor calf serum (Invitrogen) for mouse NIH 3T3 and HEK293 (293) cells, or $8 \%$ fetal bovine serum (FBS, Invitrogen) plus $250 \mu \mathrm{g} / \mathrm{ml}$ G418 (BioGold) for the H1BAG, 293/SSTR-2, $-3,-5$ and 293FT cells.

\section{3/SSTR-expressing Cell Lines}

The 293/SSTR-5 cell line was generated using plasmid Alpha+12CA5/SSTR5 (Affymax, Palo Alto, CA) and sorted as previously described to obtain a population expressing cell surface SSTR-5 at levels comparable to that of endogenous mCAT-1 on NIH 3 T3 cells [25]. The 293/SSTR-2 and -3 cell populations were generated similarly by transfection of 293 cells with expression plasmids Alpha+12CA5/SSTR2 or Alpha+12CA5/SSTR3 (Affymax) encoding amino-terminal HA epitope tagged human SSTR subtypes 2 and 3, respectively, linked to a G418 resistance gene cassette, and stable transfectants selected by culture in $1 \mathrm{mg} / \mathrm{ml} \mathrm{G} 418$ for four weeks followed by Fluorescence activated cell sorting (FACS) using mouse anti-HA monoclonal antibody HA.11 (Covance, 1:200 dilution) and goat anti-mouse conjugated to Alexa488 (Molecular Probes, 1:400 dilution) to obtain populations whose surface expression of SSTR-2 or -3 was comparable to that of the 293/SSTR-5 cell line.

\section{HIBAG Producer Cell Line}

The H1BAG cells used to produce pseudotyped MLV particles has been described previously [25]. Briefly, HEK293 cells were transfected with the BAG genome [40] and single-cell cloned to obtain a population of cells stably expressing a murine leukemia virus intact long-terminal-repeat controlled lacZ-transducing retroviral genome.

\section{Plasmid Construction}

\section{Sst-RBS and Flexible Flanking Sequence Modified Glycoproteins}

The following primer sequences were created to generate intermediate plasmids pcDNA-Sst-RBS and pcDNA-Sst-RBS-2 from pcDNA-DelRBS-Opti (a plasmid encoding a codon optimized version of the MLV env in which the RBS has been deleted) using ExSite Mutagenesis technology (Stratagene) and PrimeStar HS DNA Polymerase (Takara); DelRBS-Opti Return Primer: 5' PHOS G TCC TGG AGG ACT ACT GAA TG 3', DelRBS-Opti SstMut1 Primer: 5' PHOS CG CCT TAC GCG TCC GCT GGC TGC AAG AAT TTC TTC TGG AAG ACC TTC ACT AGT TGC GCG TAT ACC GCT AGT GAG GAG CCA CTG AC 3', and DelRBS-Opti SstMut2 Primer: 5' PHOS CT CCT AGC GGC GGT GGA GGA GCT GGC TGC AAG AAT TTC TTC TGG AAG ACC TTC ACT AGT TGC AGC GGT GGA GGA GGC GAG GAG CCA CTG ACA TCT TTG 3'. The unique restriction sites PmII and BspEI in pcDNA-DelRBS-Opti and the SpeI site in 
Sst-RBS were used to recombine the 5' and 3' flanking sequences to generate two more plasmids; pcDNA-Sst-RBS-3 and pcDNA-Sst-RBS-4. The restriction sites PmII and BspEI were again used to lift the four Sst-RBS sequences out of the pcDNA-DeIRBSOpti intermediate backbone and insert them into the TS45 expression plasmid generating Sst-RBS, Sst-RBS-2, Sst-RBS-3 and Sst-RBS-4.

\section{FEGW}

The expression plasmid encoding an HIV-based self-inactivating (SIN) LV genome encoding green fluorescent protein (GFP) under control of the human elongation factor 1-alpha (hEF1 $\alpha$ ) was created by removing the human Ubiquitin $\mathrm{C}$ promoter (hUBC) from FUGW (Addgene [41]) and replacing it with hEF1 $\alpha$ from pEF1/Myc-His A (Invitrogen) using In-Fusion (Clontech, manufacturer's instructions) between the PacI and BamHI restriction sites with the following primers. EF1a-FUGW For: TGA TTA TTG ACT AGG CTT TTG CAA AAA GCT TTG CAA AGA TGG ATA AAG TTT TAA ACA GAG AGG AAT CTT TGC AGC TAA TGG ACC TTC TAG GTC TTG AAA GGA GTG GG and Ef1aFUGW Rev: GGA ATT AGC TTG GTA CTA ATA CGA CTC ACT ATA GGG AGA CCC AAG CTG GCT AGG TAA GCT TGG TAC CGA GCT CGG ATC CAC TAG TCC AGT GTG GTG GAA TTC TGC AGA TAT CCA GCA CAG TGG CGG CCG CTC GAG TCT AGA GGG CCC TT.

\section{Pseudovirus Production}

MLV pseudovirions transducing $\beta$-galactosidase were produced by calcium phosphate co-precipitation transfection of H1BAG cells using plasmids encoding the Moloney MLV gag and pol genes (35 $\mu \mathrm{g}$ ) and either the wild type (WT) Moloney or Sst-RBS env gene $(18 \mu \mathrm{g})$ or a vesicular stomatitis virus (VSV) G protein cDNA $(4 \mu \mathrm{g})$ in a T-75 flask. The precipitant was removed and fresh growth media added after 16 hours, supernatants collected 24 and 48 hours later, and cell debris removed by $0.45 \mu \mathrm{M}$ syringe filter (Millipore). Viral supernatants were generally used fresh (no freeze-thaw), further concentrated as indicated in the experimental procedure or stored at $-80{ }^{\circ} \mathrm{C}$ for later use.

Sst-RBS coated lentiviral pseudovirions transducing enhanced GFP were produced by Lipofectamine 2000 (Invitrogen, manufacturer's instructions) transfection of 293FT cells (Invitrogen) with plasmids FEGW $(3.75 \mu \mathrm{g})$ pCMVd8.2dvpr (Addgene, $11 \mu \mathrm{g}$ ) encoding the gag, pol and rev genes from HIV-1 and the Sst-RBS env gene $(8.4 \mu \mathrm{g})$ in a T-75 flask. The VSV G LV was produced by Lipofectamine 2000 transfection of 293FT cells with FEGW or FUGW $(3.75 \mu \mathrm{g})$ and the Virapower Lentivirus plasmid mix (Invitrogen, $11.25 \mu \mathrm{g}$ ) in a T-75 flask. The transfection mix was removed and fresh growth media without phenol red added after 16 hours, supernatants collected 24 and 48 hours later, and cell debris removed by $0.45 \mu \mathrm{M}$ syringe filter. Supernatants were concentrated by ultracentrifugation at $18,000 \mathrm{rpm}$ for 3 hours at $4{ }^{\circ} \mathrm{C}$ (Beckman Coulter Optima L-90K Ultracentrifuge SW28 rotor) then resuspended in ice cold phosphate buffered saline (PBS) at 100-fold the original volume. Concentrated lentiviral stocks were used fresh (no freeze-thaw) or stored at $-80^{\circ} \mathrm{C}$ for later use. 
Lentivirus transducing mKate2, firefly luciferase and puromycin resistance were produced by the University of Tennessee Health Science Center Viral Vector Core.

\section{Infection Assays}

End-point dilution titrations of MLV pseudovirions were performed by exposing host cells seeded (at approximately $25 \%$ confluence to allow room for cell division necessary for MLV provirus integration) in quadruplicate on 48-well plates to ten-fold serial dilutions of virus supernatant in the presence of $10 \mu \mathrm{g} / \mathrm{ml}$ polybrene (Sigma) using spinoculation (centrifugation at $2250 \mathrm{rpm}$ in a Jouan CR 412 for 30 minutes at $24{ }^{\circ} \mathrm{C}$ ). Forty-eight hours later, the cells were fixed with $0.5 \%$ gluteraldehyde, stained with 5 -bromo-4chloro-3-indolyl- $\beta$-D-galactopyranoside (X-Gal) and transduction was quantified by scoring the lacZ positive foci using light microscopy.

Titration of puromycin resistance-transducing lentiviral stocks was performed similarly except that 36 hours after virus exposure, cells were fed with medium containing $1 \mu \mathrm{g} / \mathrm{ml}$ puromycin and the drug was maintained for two weeks at which time surviving drug-resistance colonies were fixed and stained for enumeration using $1 \% \mathrm{v} / \mathrm{v}$ crystal violet (Sigma) in Ethanol. For GFP-transducing LVs, host cells were seeded (at approximately $40 \%$ confluence) in a 24 -well plate and exposed to $300 \mu \mathrm{l}$ of $100 \mathrm{X}$ concentrated lentiviral (100,000 NMWL Centricon-Plus Millipore) stock resuspended at $5 X$ for Sst-RBS or $1 \times 10^{-2}$ for VSV G pseudotypes in the presence of $10 \mu \mathrm{g} / \mathrm{ml}$ polybrene. Cells were spinoculated, and split into 6-well dishes 16 hours later. Infection was quantified by flow cytometry for the presence of GFP at 72 hours post infection (hpi).

\section{SDS PAGE Western Blot Analysis}

Virus supernatants were prepared for analysis by concentrating $9 \mathrm{ml}$ by ultracentrifugation at $30,000 \mathrm{rpm}$ for 90 minutes at $4^{\circ} \mathrm{C}$ and resuspending the pellet in $30 \mu \mathrm{l}$ of ice cold PBS. Pre-cast 4-20\% Sodium dodecyl sulfate polyacrylamide gel electrophoresis (SDS PAGE) gels (Pierce) were loaded with $10 \mu \mathrm{l}$ pellet in $2 \mathrm{X}$ gel loading dye boiled at $95^{\circ} \mathrm{C}$ for 10 minutes or $5 \mu$ protein marker (Bio-Rad). The gel was electrophoresed for 15 minutes at $80 \mathrm{~V}$ then 30 minutes at $110 \mathrm{~V}$. Protein was transferred to a nitrocellulose membrane overnight at $30 \mathrm{~V}$. The membrane was probed with goat anti-Rauscher SU (Quality Biotech, 1:100 dilution) a primary antibody recognizing the MLV SU protein for 1 hour, horseradish peroxidase conjugated secondary antibody for 1 hour (Sigma, 1:5,000 dilution) and visualized with chemiluminescent substrate (Pierce). The membrane was stripped (Bio-Rad) and reprobed using goat anti-Rauscher CA (1:10,000 dilution) a primary antibody recognizing the MLV capsid protein as a loading control.

\section{Virus Binding}

Equilbrium binding assays were performed as previously described [42]. Briefly, Sst-RBS MLV was concentrated $20 X$ by low speed centrifugation using Centricon-100 devices (Millipore) then incubated with approximately $1 \times 10^{6}$ non-transfected 293 or 293/SSTR-2, -3 , or -5 cells for 30 minutes at $37^{\circ} \mathrm{C}$ to allow maximal virus particle binding. After washing out unbound virus with PBS the cells were incubated for 1 hour at 
$4{ }^{\circ} \mathrm{C}$ with goat anti-Rauscher SU (Quality Biotech, 1:100 dilution) cleared by pre-incubation with 293 cells and for 30 minutes with mouse anti-goat Alexa-488 (Molecular Probes, 1:400 dilution). Finally, $2 \mu \mathrm{g}$ of propidium iodide (Sigma) added to gate out membrane compromised cells. Flow cytometry of at least 10,000 events was performed and the population of propidium iodide -negative, Alexa-488-positive cells was analyzed. The fold binding of Sst-RBS MLV to each cell line was determined by comparing the mean fluorescent intensity (MFI) of each SSTR-expressing cell line exposed to Sst-RBS MLV to the MFI of non-exposed populations (this normalizes for background fluorescence).

\section{Receptor Internalization}

Receptor internalization studies were performed similarly to the equilibrium virus binding assay but with the following exceptions: incubation with $1 \mu \mathrm{M}$ of synthetic SST-14 peptide (Sigma, S-9129) for 30 minutes followed by incubation for 1 hour at $4{ }^{\circ} \mathrm{C}$ with mouse anti-HA.11 monoclonal antibody (Covance, 1:200 dilution) and goat anti-mouse conjugated to Alexa488 (Molecular Probes, 1:400 dilution).

\section{Kinetics of Virus Internalization}

To determine the internalization kinetics viruses were spinoculated onto host cells seeded in quadruplicate wells of 48-well plates by centrifugation at $2250 \mathrm{rpm}$ (Jouan CR 412) for 30 minutes at $4{ }^{\circ} \mathrm{C}$ in the presence of $10 \mu \mathrm{g} / \mathrm{ml}$ polybrene, after which non-internalized virions were inactivated by a pH 3.0 citrate buffer wash immediately ( 0 hour time point) or at various times after shifting the cells to $37^{\circ} \mathrm{C}$ as previously described [43]. Infection was allowed to continue for 48 hours prior to fixation with $0.5 \%$ gluteraldehyde and stained with X-Gal. $\beta$-galactosidase activity was quantified using the Romanizer software package ((http://cfar.ucsd.edu/romanizer/) as described by Day and coworkers [44] except that the digital images of whole fixed and stained wells were captured using a D50 digital camera (Nikon) mounted on a SZ-PT microscope (Olympus) by Tim Higgins in the Department of Microbiology, Immunology and Biochemistry. In a pilot experiment, infected 293/SSTR-5 and NIH 3T3 cells were too numerous to count accurately either by visual scoring using light microscopy or using the Romanizer software so in the experiments reported here these cell lines were spinoculated with 100-fold dilutions of the Sst-RBS or WT MLV, while the 293 and 293/SSTR-2 and -3 host cells were spinoculated with neat Sst-RBS MLV stocks. The relative rate of virus internalization was calculated by non-linear regression analysis using GraphPad Prism software and the amount of time to reach $50 \%$ infection extrapolated from each equation.

\section{Drug and Inhibitor Studies}

Stock solutions were prepared as follows: $500 \mathrm{mM}$ methyl- $\beta$-cyclodextrin (Sigma, C4555), $30 \mathrm{mM}$ chloroquine diphosphate salt (Sigma), $5 \mathrm{mg} / \mathrm{ml}$ leupeptin hemisulfate (Peptides International) in $\mathrm{H}_{2} \mathrm{O}, 3 \mathrm{mM}$ monensin in $\mathrm{EtOH}$ (ready to use as GolgiStop, $\mathrm{BD}$ ) and $10 \mathrm{mM}$ CA074 Me (Peptides International), $10 \mathrm{mM}$ nocodazole (Sigma), $20 \mathrm{mM}$ bafilomycin A1 (Sigma) in DMSO. Cells seeded in quadruplicate wells on 96-well plates were pretreated with medium containing the indicated concentration of each compound 
or vehicle for 30 min (methyl- $\beta$-cyclodextrin; bafilomycin A1, chloroquine, monensin and CA074 Me) or 1 hour (leupeptin and nocodazole) at $37^{\circ} \mathrm{C}$, then virus was spinoculated as described above except that virus plus drug or vehicle was maintained for 3 hours after spinoculation, then unbound virus and drugs were removed by two gentle washes with culture medium. For cells treated with methyl- $\beta$-cyclodextrin, each well was rinsed twice with Optimem I serum-free medium and methyl- $\beta$-cyclodextrin or vehicle was also diluted in Optimem I prior to its addition to adherent cells. At $48 \mathrm{hpi}$, cells were fixed and stained with X-Gal and transduction was scored using the Romanizer software to analyze digital images of entire wells. In order to obtain levels of infection that could be accurately quantified, 1:20 dilutions of control VSV G MLV were used on all cell lines and the same dilutions of Sst-RBS and WT MLV on 293/SSTR-5 and NIH 3T3 cells, respectively. The amount of infection in the vehicle-only control wells was taken as $100 \%$ infection and the percent infection of each cell line was calculated for each experiment individually to determine the mean percent infection in the quadruplicate wells.

\section{Quantitative Real-time PCR Analysis}

The quantitative real-time PCR assays were performed as previously described by Gao and colleagues [45] with the following modifications. New primer sets were developed for the MSS and lacZ of the recombinant MLV genome, and for human and murine $\beta$-actin control amplicons. Three-fold concentrated Sst-RBS or WT MLV pseudovirions, or mock (no virus) were spinoculated onto the cells by centrifugation at $2250 \mathrm{rpm}$ (Jouan CR 412) for $30 \mathrm{~min}$ at $4{ }^{\circ} \mathrm{C}$ in the presence of $10 \mu \mathrm{g} / \mathrm{ml}$ polybrene, then unbound virus was washed away and the cells fed fresh medium. Whole cell DNA was isolated immediately with no temperature shift for the zero time point, and replicate samples were also shifted to $37^{\circ} \mathrm{C}$ for 6 hours prior to DNA isolation using a DNeasy Blood and Tissue Kit (Qiagen). The whole cell DNA was used directly as template for analysis by quantitative real-time PCR using the Light Cycler 480 instrument (Roche) and the following primer and probe sets: Minus Strand Strong Stop (MSS), forward (MSS82-F) 5'-ACT TGT GGT CTC GCT GTT CC-3', reverse (MSS82-R) 5'-CGC TGA CGG GTA GTC AAT C-3' and Universal Probe \#82 (Roche); LacZ gene, forward (LacZ74-F) 5'-GAC CGC ATG GTC AGA AGC-3', reverse (LacZ74-R) 5'-CTG AGG TTT TCC GCC AGA C-3' and Universal Probe \#74; Human $\beta$-actin gene, forward (hBAct60F) 5'-GCC GTG TTC TTT GCA CTT TC-3', reverse (hBAct60-R) 5'-GCA GAG ATG CAC CAT GTC AC-3' and Universal Probe \#60; murine $\beta$-actin gene: forward (mBAct29-F) 5'-CAC GCC CTT TCT CAA TTG TC-3', reverse (mBAct29-R) 5'-CCA AGG GAG ACT CAG CTC ATA-3' and Universal Probe \#29. MSS and LacZ gene amplification were normalized to the values obtained for $\beta$-actin amplification for each respective host cell line. The relative amount of cell-associated virus was calculated by comparing the MSS amplification signal at the 6 hour time point for each SSTR-expressing cell lines to the level in the parent 293 cells using the delta delta Ct calculation. Values were then expressed as the relative fold increase over the 293 value. To determine the relative amount of penetrated virus the delta delta $\mathrm{Ct}$ calculation was used to calculate the relative level of MSS signal at the zero and 6 hour time points with respect to the level of LacZ gene signal in each cell line, and then expressed as the fold increase over the zero hour time point. 


\title{
In Vivo Experiments
}

\begin{abstract}
Animals
The animal protocol used in this study has been approved by the Institutional Animal Care and Use Committee at the University of Tennessee Health Science Center (UTHSC). Nine to twelve week old C57BL/6J mice were purchased from Charles River Laboratories (Wilmington, MA, USA). Mice were allowed free access to food and water.
\end{abstract}

\section{Intracranial Microinjection}

Bilateral, stereotaxic microinjections were performed on anesthetized mice using the following protocol. Nine to twelve week old C57BL/6 mice skulls were shaved and incision site prepped with betadine then mice were mounted into a stereotaxic apparatus. Small holes were drilled in the skull bone directly above the injection sites and a 30-gauge Hamilton microsyringe lowered to the following coordinates from bregma: Caudate Putamen: $\mathrm{AP}=-0.5, \mathrm{ML}= \pm 2.0, \mathrm{DV}=-4.0$; Subthalamic Nucleus: $A P=-1.9, M L= \pm 1.5, D V=-5.2$. After the microsyringe had been lowered to the coordinates, it was left in place for 10 minutes before and after administration of lentiviral injections. A total volume of $2 \mu \mathrm{l}$ concentrated Sst-RBS or a 100 -fold dilution of concentrated VSV G pseudotyped lentiviral vectors transducing a SIN HIV-1 based genome encoding the marker gene mKate 2 under the $\mathrm{hUbC}$ promoter were injected into the left hemispheres of 4 mice at a rate of $0.7 \mu \mathrm{l} / \mathrm{min}$. The same virus preparations were also injected into the contralateral hemisphere of each mouse immediately after injection with $0.5 \mu \mathrm{l}$ of $100 \mu \mathrm{M}$ SST-14 peptide. After the injection procedure was complete, the skull incision was closed and mice were allowed to recover in a BSL-2 housing facility. Three days post infection the mice were sacrificed, organs perfused with PBS and the brain tissue recovered for analysis.

\section{Tissue Section Staining}

Brain tissue from injected and control mice were fixed in $4 \%$ paraformaldehyde then sunk in a $25 \%$ sucrose phosphate buffer solution and flash frozen in Isopentane. A microtome was use to slice $20 \mu \mathrm{m}$ sections which were placed on clean, glass slides. Slides were soaked in 1X PBS to remove the sucrose solution from the sections just prior to staining to allow antibodies proper access to epitopes. Sections were blocked for 20 minutes with $3 \%$ BSA/ 0.8\% Triton X-100/ 0.2M PBS prior to co-incubation with monoclonal rabbit anti-SSTR-2 (Epitomics, 1:1000 dilution) and monoclonal mouse anti-neuronal nuclear membrane protein (NeuN) (Millipore, 1:2000 dilution) diluted in blocking buffer overnight followed by incubation with Alexa-488, Alexa-594 or Alexa-647 conjugated secondary antibodies (Molecular Probes, 1:1000 dilution) for 2 hours. Hoechst nuclear stain $(100 \mu \mathrm{M})$ was added to stained sections for 20 minutes prior to mounting in $90 \%$ glycerol/PBS and imaged on a Zeiss Deconvolution microscope or in FluorSave (Calbiochem) and imaged using an Aperio Scanscope. 


\section{Transduction Zone Measurements}

The mean transduction area (TA) of multiple sections from three mice injected with $2 \mu \mathrm{l}$ of either the Sst-RBS or VSV G pseudotyped LV vectors was quantified using ImageJ software (National Institutes of Health, v1.46r). Transduction of mKate2 was analyzed for each coronal section. These values were then used to calculate a mean TA for individual mice and an overall mean TA for each lentiviral vector.

The mean transduction zone (TZ) of multiple sections from three mice injected with $2 \mu \mathrm{l}$ of either the Sst-RBS or VSV G pseudotyped LV vectors, in the presence or absence of $100 \mu \mathrm{M}$ SST-14, was quantified by measuring the height (h) and width (w) of the core and maximum TZ using ImageScope software (Aperio, v11.2.0.780) and calculating the elliptical area using the formula $a=\pi \mathrm{hw} / 4$. The core $\mathrm{TZ}$ was defined as the area with the highest concentration of mKate 2 expression and the maximum TZ as the largest overall area of mKate2 expression. Mean TZ areas were calculated for individual mice which were then used to calculate an overall mean TZ area for each lentiviral vector.

\section{Statistical Analysis}

Descriptive statistics for all data were performed using Prism GraphPad Software v4.0. 


\section{Chapter 3. Targeted Entry via Somatostatin Receptors Using a Novel Modified Retrovirus Glycoprotein that Delivers Genes at Levels Comparable to Those of Wild-Type Viral Glycoproteins ${ }^{\dagger}$}

\section{Introduction}

Retro- and lentivirus vectors are commonly used as vehicles for marker and therapeutic gene delivery because they naturally integrate a copy of their genomic material into the host chromosome during infection. Generally, these vectors use the Env glycoprotein from amphotropic MLV or the VSV G protein. Unfortunately, the broad tropism of these glycoproteins mediates gene transduction of most cells after in vivo injection, resulting in the need for a far greater vector inoculum than would be required if entry could be restricted to the target cells or tissues capable of benefiting from therapeutic gene delivery. This is one of the most significant challenges to targeted in vivo gene delivery [13].

Addressing the need for targeted delivery in vivo, a number of approaches based on RV and LV particles have been reported (for reviews see [11, 12]). Placing glycoproteins modified with ligand insertions directed to cellular receptors onto virus particles (pseudotyping) is a common strategy. Although these chimeric virions were generally able to bind their receptors and internalize, membrane fusion was impaired resulting very low levels of transduction [13-15]. Since Env conformational changes are normally activated by interactions of the Env RBS with its cellular receptor to initiate membrane fusion, a new strategy was proposed to replace the MLV RBS with a peptide ligand.

Other researchers in this laboratory demonstrated that a chimeric MLV Env glycoprotein based on this design, Sst-RBS (Figure 1-1), was capable of infecting 293 cells expressing the SSTR-2 receptor and no longer used its natural receptor, mCAT-1. This infection was found to be SSTR-specific as evidenced by dose-dependent inhibition of infection using exogenous SST-14 peptide [25]. The strategy used in designing the Sst-RBS glycoprotein should mimic natural virus-receptor interactions as the Sst-RBS Env binds to its target receptor and be capable of inducing membrane fusion, thus allowing targeted transduction. It was demonstrated that an important positional requirement for the glycoprotein was satisfied by placing the SST-14 sequence at the RBS. In this chapter I report that an additional glycoprotein characteristic, conferred by specific structural properties of the Sst-RBS replacement sequence, influences infection. Additionally, the transduction capacity of pseudovirions bearing the Sst-RBS glycoprotein was comparable to the efficiency of natural retroviral glycoproteins and approached that of VSV G; establishing Sst-RBS as a suitable candidate for in vivo gene transfer.

${ }^{\dagger}$ Adapted with permission. Li, F., B. Y. Ryu, R. L. Krueger, S. A. Heldt and L. M. Albritton. Targeted entry via somatostatin receptors using a novel modified retrovirus glycoprotein that delivers genes at levels comparable to those of wild-type viral glycoproteins. J Virol, 2012. 86(1): 373-381. 


\section{Results}

\section{Sst-RBS Is Capable of Transducing 293/SSTR-5 Cells at Levels Comparable to Wild-type Transduction of NIH 3T3 Cells}

To begin investigating Sst-RBS transduction capacity, I compared its transduction with WT and VSV G pseudotyped MLV particles by end-point dilution titration (Figure 3-1A). In four independent titrations, the mean LacZ transducing units per ml of Sst-RBS reached $5.6 \times 10^{5}$ (range of $2.5 \times 10^{5}-1.0 \times 10^{6}$ ) in SSTR-5 expressing cells, WT reached $5.8 \times 10^{5}$ (range of $2.5 \times 10^{5}-1.0 \times 10^{6}$ ) in NIH 3T3 cells expressing the natural receptor and VSV G reached $1.1 \times 10^{6}$ (range of $1.0 \times 10^{5}-2.5 \times 10^{6}$ ) in 293 cells (Figure 3-1B).

\section{Pseudovirion Binding to SSTR-5 Is Comparable to mCAT-1}

When comparing the level of infection of the Sst-RBS glycoprotein to that of WT, it is important to maintain comparable numbers of available binding sites on each of the cell lines. To do this, a binding assay was performed with both pseudovirions. This assay is described in detail in the virus binding assay section of Chapter 2. Briefly, 293/SSTR-5 cells and NIH 3T3 cells were incubated with the appropriate virus and levels of attachment were quantified by flow cytometry using antiserum to the MLV SU Env (Figure 3-2A). The mean fluorescent intensity (MFI) of the bound virus can be used as a measure of the relative number of pseudovirion binding sites since the anti-SU antiserum appeared to recognize the Sst-RBS and WT Env similarly in immunoblots [25]. In three independent assays, Sst-RBS MLV showed a mean increase of $2.9 \pm 0.7-$ fold in binding to SSTR-5 expressing cells over cells incubated with antibody alone and WT MLV showed a mean increase of $4.3 \pm 0.2$-fold. Histograms from a representative assay are shown in Figure 3-2B. Because these receptors are multi-membrane spanning proteins virus binding kinetics cannot be accurately determined; however, the difference in the level of virus binding to these cells indicates that the binding of WT to the murine cell line NIH 3T3 is only slightly greater than the capacity for Sst-RBS binding to SSTR-5 expressing cell. Thus, the effective number of receptor binding sites can also be considered comparable assuming that the receptors display similar off-rates.

\section{A Non-flexible Turn Flanking the SST-14 Ligand Insertion Is Required for Sst-RBS Infectivity}

A primary reason for the failure of many Env retargeting designs can be attributed to improper structural relationships and protein folding. Incorrectly folded glycoproteins might not be incorporated into the viral membrane or fail to complete membrane fusion because critical conformational changes are impeded [11, 46]. Replacing the natural RBS minimizes this concern by leaving critical residues in the amino-terminus free [8], however steric hindrances may still exist that prevent efficient transduction. As such, it has been shown that the addition of flexible peptides might alleviate these constraints $[14,47]$.

To determine if structural requirements of the Sst-RBS glycoprotein have an effect on transduction capacity, mutagenic PCR techniques were used to alter the Sst-RBS insertion sequence by replacing the amino- or carboxy-flanking sides of the SST-14 
A

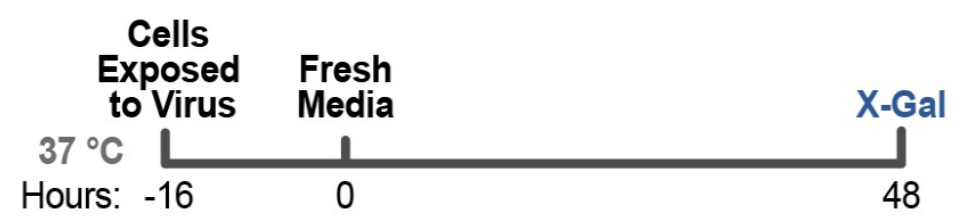

B
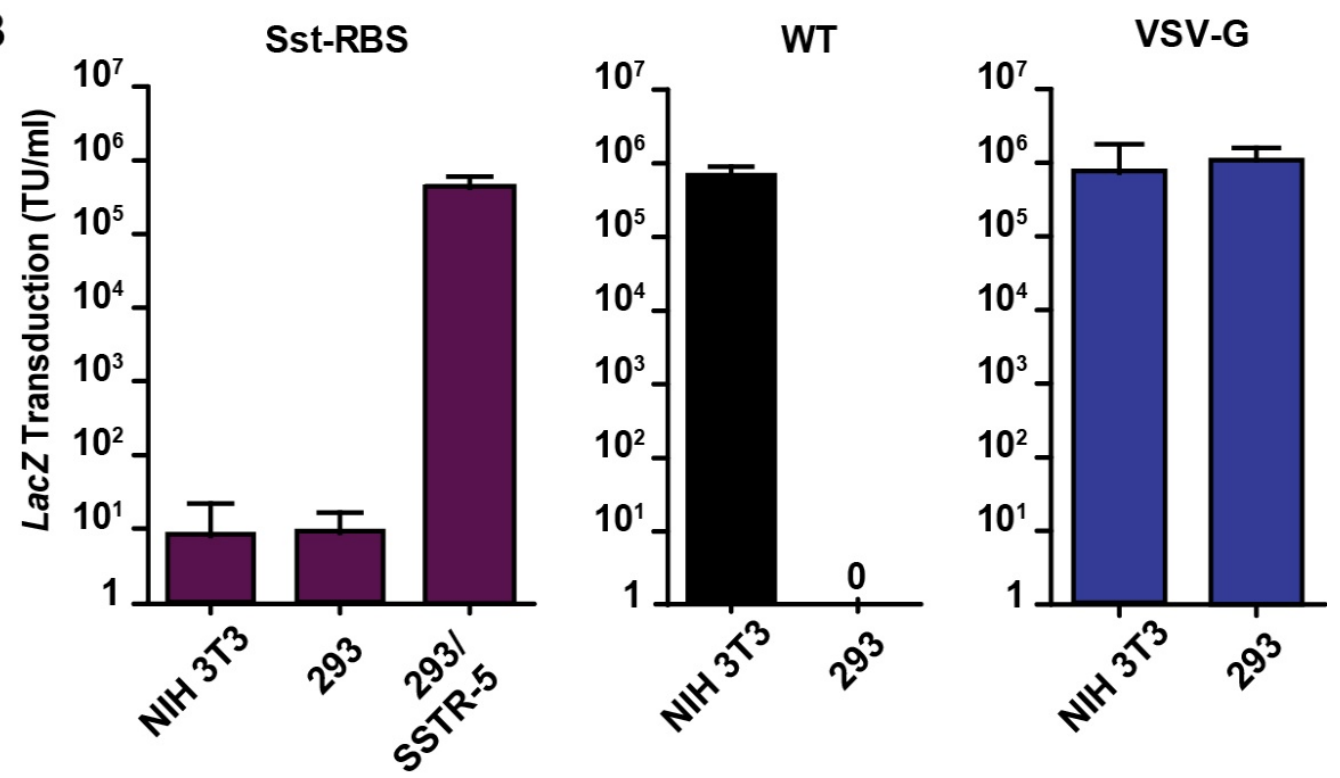

Figure 3-1. Sst-RBS glycoprotein targets entry at levels comparable to those of entry mediated by wild-type Moloney murine leukemia virus glycoprotein and approaching those of VSV G pseudovirions
A. Experimental time line for transduction assays.
B. NIH 3T3, 293, or 293/SSTR-5 cells were exposed to 10-fold serial dilutions of independently produced MLV vectors pseudotyped with the Sst-RBS (left) WT (middle) or VSV G (right) envelope glycoprotein and the titers were calculated from the endpoint dilutions. Data represent the mean \pm SD of 3-4 independent titrations $(n=4)$. 
A

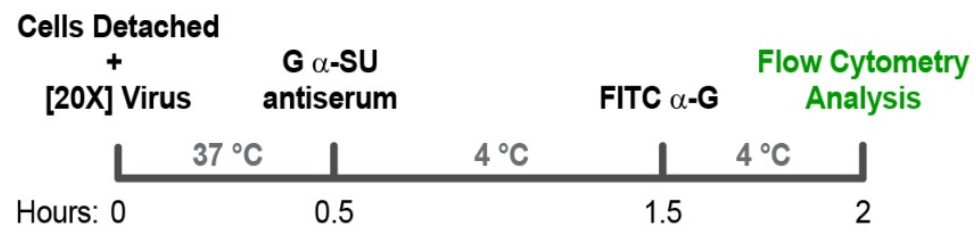

B
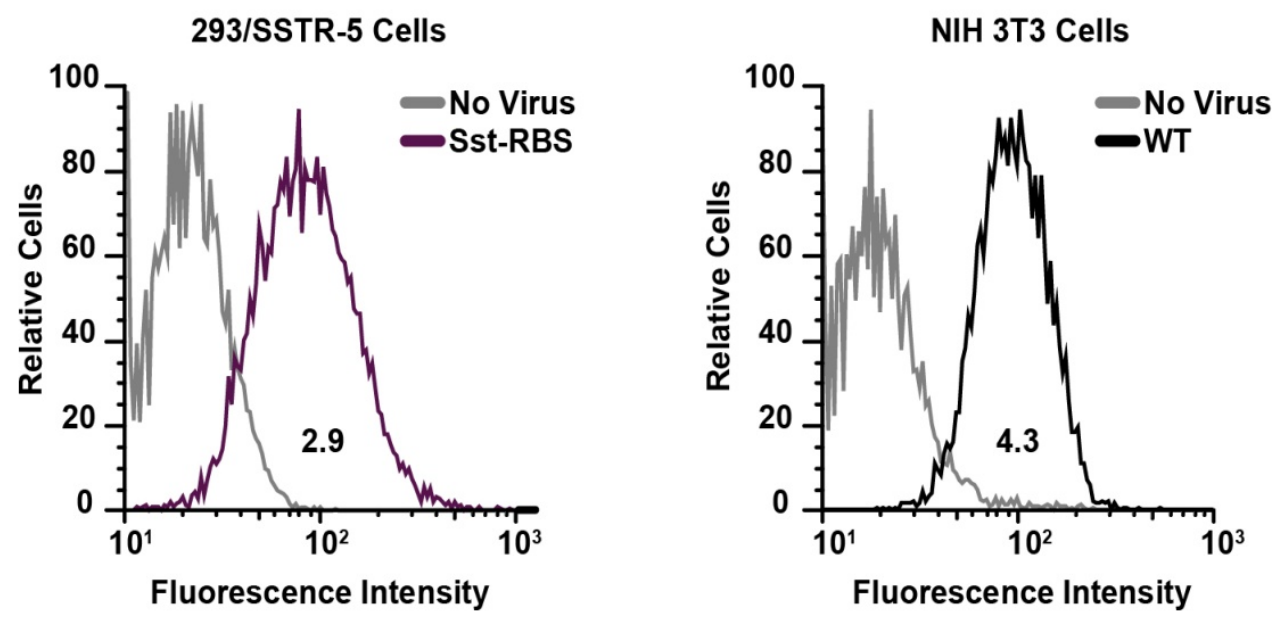

Figure 3-2. Generation of a host cell line stably expressing levels of target receptor SSTR comparable to the levels of ecotropic receptor on NIH 3T3 cells
A. Experimental time line for virus binding assay.
B. 293 cells were transfected with a cDNA-encoding human SSTR-5 with an amino- terminal HA epitope tag, selected for G418 resistance and further selected for surface expression of the target receptor by three rounds of FACS using anti-HA antibody, yielding a population of cells designated 293/SSTR-5 .The 293/SSTR-5 (left) or NIH 3T3 (right) cells were incubated with Sst-RBS or WT pseudovirions or mock incubated (No virus), and then both samples were incubated with goat anti-SU antiserum followed by a mouse anti-goat Alexa488-conjugated secondary antibody plus propidium iodide at the temperatures indicated in the experimental time and analyzed by flow cytometry. The histograms shown are representative of the results of three independent binding experiments. Values shown represent the mean fold increase in Alexa488 intensities determined with live (propidium iodide-negative) cells. 
ligand with the flexible residues SGG or SGGGG, respectively (Figure 3-3). End-point dilutions were performed using pseudovirions coated with these glycoproteins. The mean titers of three independent titrations of the flexible linker-modified viruses obtained from 293/SSTR-5 cells are presented in Figure 3-4A. Modifying the flanking sequences of Sst-RBS did not improve infection and, in fact, placing a flexible sequence before the SST-14 ligand had a deleterious effect. Only the level of infection of Sst-RBS-3 was comparable to the original Sst-RBS construct and levels of WT infection on NIH 3T3 cells. Infection of Sst-RBS-2 and Sst-RBS-4 were both decreased.

SDS-PAGE western blot analysis was performed on concentrated pellets obtained from supernatants of WT, Sst-RBS and Sst-RBS-2 through 4 and probed with anti-SU antiserum. The MLV capsid (CA) protein served as a loading control since each virus particle contains approximately the same amount of this protein. The SU and CA band intensities were quantitated and the intensity of each SU band was normalized to the amount of respective CA (Figure 3-4B). Sst-RBS displayed a band intensity that was only slightly lower than the intensity of the WT band. In contrast, Sst-RBS-2, -3 and -4 had reduced band intensities indicating that the modifications to these glycoproteins caused decreases in their incorporation into the viral membrane possibly because the glycoproteins were unable to fold into the correct conformation.

\section{Sst-RBS Pseudotyped Lentiviral Vectors}

To further characterize the usefulness of Sst-RBS, HIV-based SIN lentiviral vectors transducing the far-red fluorescent protein mKate2 and firefly luciferase were pseudotyped with Sst-RBS (Sst-RBS LV) or VSV G (VSV G LV), concentrated and certified replication incompetent by the UTHSC Viral Vector Core. Transducing units (TU/ml) of these vectors were quantified by end-point dilution titration on 293/SSTR-5 and 293 cells using a standard luciferase assay by the vector core. The VSV G LV stock

\begin{tabular}{cc} 
Glycoprotein & Sequence Alignment \\
\hline Octreotide: & FCFWKTCT \\
SST-14: & AGCKNFFWKTFTSC \\
WT MLV: & GPP---CCSGGSSPGCSRDC-----EEP \\
Sst-RBS: & GPPYASAGCKNFFWKTFTSCAYTASEEP \\
Sst-RBS-2: & GPPSGGAGCKNFFWKTFTSCAYTASEEP \\
Sst-RBS-3: & GPPYASAGCKNFFWKTFTSCSGGGEEP \\
Sst-RBS-4: & GPPSGGAGCKNFFWKTFTSCSGGGEEP
\end{tabular}

Figure 3-3. Modified Sst-RBS glycoproteins containing the SST-14 ligand

BOLD text indicates linker sequences, italic text indicates disulfide bonded cysteine residues, underlined text indicates SST-14 replacement sequence, and highlighted text indicates residues required for receptor binding. 
A
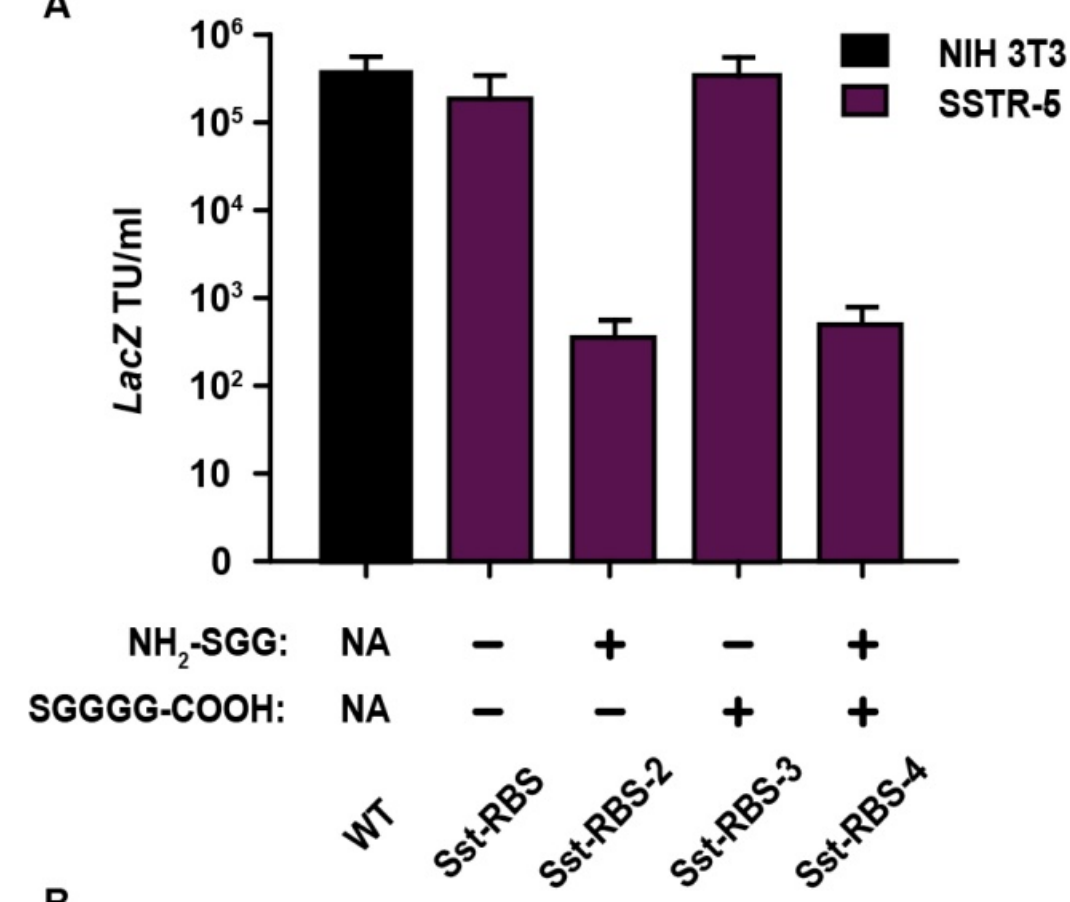

B

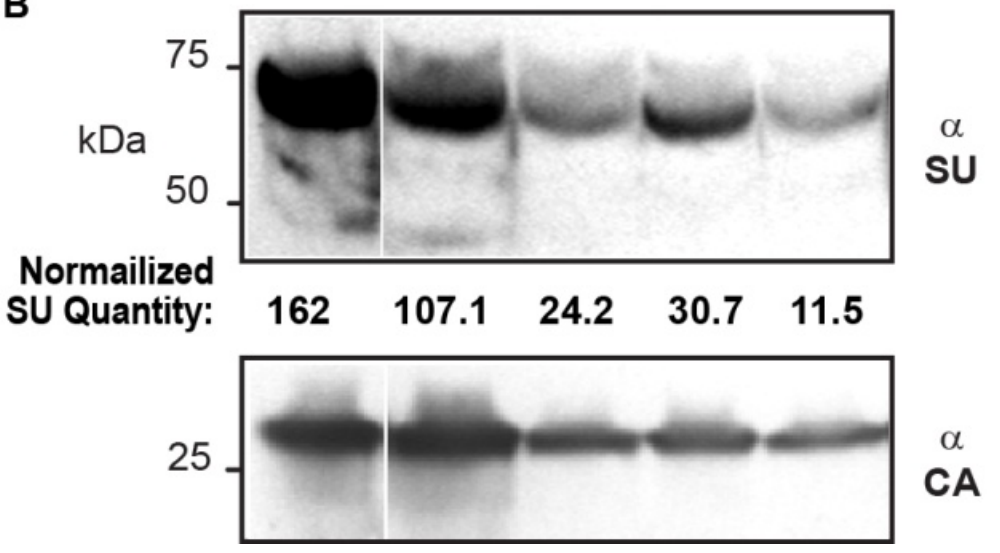

Figure 3-4. Glycoproteins with a non-flexible peptide linker sequence upstream of the SST-14 ligand retain infectivity

A. Ten-fold dilutions of MLV virions coated with Sst-RBS, the flexible-linker modified SstRBS-2, -3 , and -4 (purple) or WT (black) glycoproteins were exposed to 293/SSTR-5 or $\mathrm{NIH} 3 \mathrm{~T} 3$ cells and infection scored by LacZ transduction (unpublished data). The presence or absence of amino- and carboxy-flanking flexible residues is indicated under the bars corresponding to each pseudovirus. Data represent the mean \pm SD of 3 independent titrations $(\mathrm{n}=4)$.

B. SDS PAGE Western Blot was performed on concentrated virus pellets from a representative production of the pseudovirions in (A) (unpublished data). The membrane was probed with goat-anti p70/SU recognizing the MLV glycoprotein, stripped and reprobed with goat-anti p30/CA recognizing the MLV capsid protein as an indication of the overall virus particles present in each sample. 
contained $2.3 \times 10^{9}$ luciferase TU/ml and the Sst-RBS LV stock contained $9.2 \times 10^{6}$ luciferase $\mathrm{TU} / \mathrm{ml}$. This represents an approximate 200 -fold difference in titer between VSV G and Sst-RBS LV. This is similar to titers of other retroviral glycoprotein pseudotypes of lentiviral vectors and is thought to result from lower assembly of other glycoproteins compared to VSV G due to their cytoplasmic tail sequences [23, 48, 49]. Sst-RBS and VSV G LV particles transducing GFP under the control of the hUbC promoter (Figure 3-5A) were produced in an effort to detect transduction by flow cytometry analysis. In accordance with the difference in luciferase TU/ml, the MFI of VSV G LV transduced 293/SSTR-5 cells was detected well above the background MFI of mock exposed cells while Sst-RBS LV transduction could not be detected. When the promoter element of this HIV-based genomic construct was replaced with the hEF1 $\alpha$ promoter, the MFI of both constructs increased so that Sst-RBS transduction of SSTR-5 expressing cells was detectable; however, VSV G transduction was 20.2-fold higher (Figure 3-5B).

\section{Discussion}

Many RV and LV receptors are multiple membrane spanning proteins such as the chemokine co-receptors used by HIV [50], Xpr1 used by xenotropic MLV [51] and mCAT-1, the natural Moloney MLV receptor [52]. Although SSTR are not entry receptors for any known virus they also have multiple membrane spanning domains. Conversely, the epidermal growth factor (EGF) receptor (ErbB) has a single transmembrane domain. Chimeric virions containing an insertion of EGF in amphotropic [53] or Moloney [54] MLV Envs were capable of binding ErbB but no transduction was seen. These observations lead both Cosset and Katane's groups to propose that receptor characteristics are the main consideration for developing an entry targeted viral vector, possibly because receptors that span the cellular membrane multiple times would bring bound virus into close contact with the cell [54]. Additionally, polytopic receptors such as these are often localized in cholesterol-rich lipid rafts which are crucial for the endocytosis and entry of many of enveloped viruses [55].

Research conducted while characterizing the Sst-RBS glycoprotein demonstrates that properties of the targeting glycoprotein are also an important parameter. Viral glycoproteins naturally assemble onto virions in a metastable conformation that is not competent for membrane fusion. In the case of gammaretroviral Env, the transition from a metastable to a fusion competent conformation is induced after receptor binding and begins with cleavage by host cell cathepsin proteases in the late endosomal compartment which subsequently triggers isomerization of the disulfide bond between SU and TM domains of Env [56]. These conformational changes expose the TM fusion peptide that is responsible for completing the membrane fusion process [57]. It follows that the Env sequences that bind natural retrovirus receptors possess the ability to initiate and sustain these critical conformation changes. This capacity most likely derives from the position of the RBS at the top of the receptor binding domain, and while this location may not be the only one with the capacity to initiate and sustain membrane fusion, it is the most effective one discovered to date [25]. Furthermore, the low level of LacZ transduction observed with Sst-RBS-2 and -4 coated viruses correlated with low levels of anti-SU probe signal in a western blot analysis (Figure 3-4), indicating that placing flexible sequences in this important region affected glycoprotein incorporation into the virion envelope. It is interesting that Sst-RBS-3 displayed a similar SU band intensity as Sst-RBS-2 and -4 but retained the same level of transduction as the original 
A

\begin{tabular}{|c|c|c|c|}
\hline FUGW & 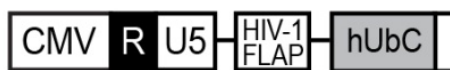 & EGFP & WRE $\triangle \cup 3 \quad R \quad U 5$ \\
\hline & CMV R U5 & EGFP & \\
\hline
\end{tabular}

B
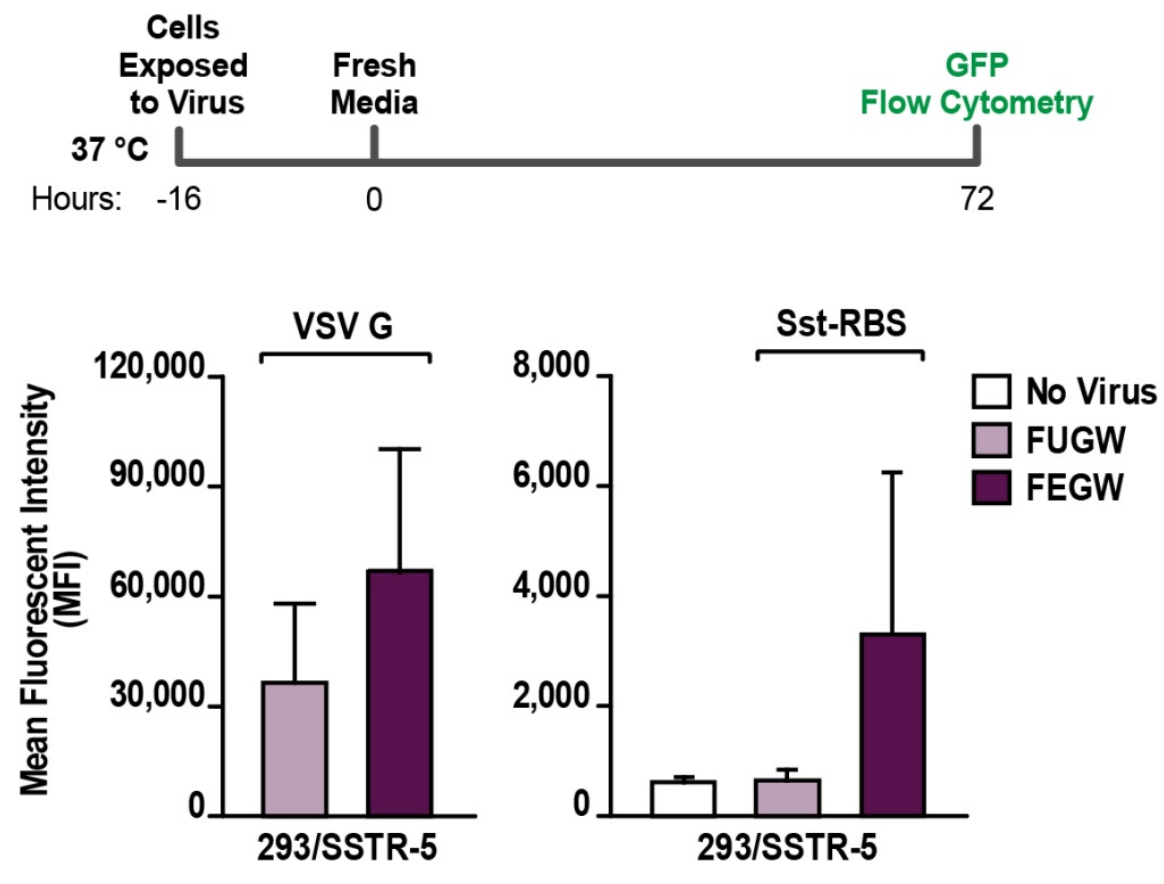

Figure 3-5. Lentivirus transducing an EF1 $\alpha$-GFP transgene exhibits higher expression

A. Schematic representation of the HIV-1 based SIN-lentiviral expression genomes. Both plasmids contain a CMV enhancer element substituted for the U3 region of the 5' LTR and a deleted 3' U3 region rendering the 5' LTR of the integrated provirus inactive. FUGW (top) transduces a GFP reporter sequence under the human Ubiquitin C promoter (hUbC) and FEGW (bottom) transduces GFP under the human Elongation Factor 1-alpha promoter (hEF1 $\alpha$ ). R, repeat region; U5, unique 5' untranslated region; HIV-1 FLAP, enhances titer by increasing cDNA nuclear import; EGFP, enhanced green fluorescent protein; WRE, woodchuck hepatitis posttranscriptional regulatory element; $\Delta \mathrm{U} 3$, unique 3 ' untranslated region deletion.

B. 293/SSTR-5 cells were exposed to mock (white bars) or unconcentrated VSV G (left panel) or Sst-RBS (right panel) coated lentiviral vectors transducing either FUGW (light bars) or FEGW (dark bars) and assayed by flow cytometry analysis 72 hours post infection for GFP fluorescence at the times indicated in the experimental timeline. 
Sst-RBS glycoprotein. Sst-RBS also exhibited a decrease in SU band intensity compared to WT which did not significantly affect its relative level of transduction. This suggests that the rigid structure provided by the original Sst-RBS insertion sequence may be a necessary structural element and it is this property, not the overall level of glycoprotein incorporation, which influenced the levels of transduction.

These data strongly indicate that the chimeric Sst-RBS Env retains the structural characteristics that are necessary to initiate infection. Despite slightly lower levels of cell surface binding sites (Figure 3-2) and Sst-RBS Env detection by WB analysis (Figure 3-4) and compared to WT, the Sst-RBS glycoprotein was capable of specifically delivering genes to cells expressing the SSTR-5 receptor with a transduction efficiency comparable to WT virus and approaching that of the "gold-standard" VSV G protein (Figure 3-1) demonstrating that the capacity of Sst-RBS MLV was within the range of a typical transduction vector. However, the difference in the level of transduction using an HIV-based LV vector pseudotyped with Sst-RBS or VSV G was larger than the difference seen from MLV particles pseudotyped with these Envs (Figure 3-5). The ability to transduce non-dividing cells is a distinct advantage of using LV vectors in in vivo systems. The influence of the 100-fold lower relative transduction capacity of SstRBS LV compared to Sst-RBS coated MLV-based vectors as measured in cultured host cells would likely be outweighed by the mitotic requirement for MLV integration. There is a need for an in vivo targeting vector that is capable of directing high capacity transduction to particular cell or tissue types of interest. This chapter demonstrated that, in addition to the position of the targeting ligand, the structure of Sst-RBS glycoprotein allowed a high level of transduction. Hence, the Sst-RBS glycoprotein is a suitable candidate for mediating in vivo targeted transduction. 


\section{Chapter 4. Characteristics of the Cellular Receptor Influence the Intracellular Fate and Efficiency of Retrovirus Infection ${ }^{\ddagger}$}

\section{Introduction}

Viral glycoproteins and their host cell entry receptors are thought to have co-evolved [58]. Data presented in Chapter 3 and published previously [25] highlighted the importance of glycoprotein characteristics on the efficiency of Sst-RBS-mediated transduction. While specific traits of the viral glycoproteins likely represented a major selective pressure driving the choice of receptor during that co-evolution [59], characteristics of candidate host cell receptors are also suspected of being involved but their possible influence has been difficult to assess [16, 60]. Virus-receptor complexes are internalized by existing endocytic mechanisms such as classical clathrin-mediated, caveolar/lipid raft associated, macropinocytosis, and phagocytosis [55, 61]. After budding from the plasma membrane, vesicles formed by these endocytic mechanisms become early endosomes (EE). As EEs mature into late endosomes (LE), the pH of their intralumenal environment decreases from physiological $\mathrm{pH}$ to as low as 5.0. Maturation is a dynamic process in which endocytosed cargo is sorted, numerous intralumenal vesicles are formed and various types of recycling vesicles bud off under the direction of numerous Rab GTPases and their associated effector molecules. During this process, the contents of endosomes are acted on by multiple cellular proteases that become activated as the $\mathrm{pH}$ decreases. LEs eventually fuse with lysosomes where the $\mathrm{pH}$ drops even further sustaining a highly acidic and proteolytically degradative environment [62]. Several pieces of circumstantial evidence suggest that the fate of internalized virusreceptor complexes can strongly influence infection outcomes and thereby could have exerted selective pressure on the choice of a cellular receptor during evolution of viral glycoproteins. First, the Ebola virus fusion receptor (Neimann-Pick C1, NPC1) and activating host cell protease (cathepsin $\mathrm{B}$ ) reside in a specific intracellular compartment $[63,64]$, suggesting that only filoviruses internalized into that compartment undergo efficient, productive entry. Second, inhibiting lysosomal degradation using pharmacologic agents increased infection by HIV-1 and the betaretrovirus enzootic nasal tumor virus (ENTV) [65-67], suggesting that internalization into degradative compartments can negatively impact the efficiency of entry. Third, specific inhibition of host cell cathepsin B increased infection of VSV G pseudotyped MLV [7] further suggesting that certain degradative compartments can be less productive destinations for entry. Lastly, prolonged neutralization of intracellular compartments decreased infection of the alpharetrovirus avian sarcoma and leukosis virus subgroup A (ASLV-A) [68] and re-routed virus-receptor complexes to different compartments [69], suggesting that the alternative compartments were less permissive than the compartment to which the virus normally traffics.

The studies reported in this chapter took advantage of the unique opportunity provided by the Sst-RBS modified Env and its capacity to transduce cells by multiple cellular receptors to directly interrogate the influence of intracellular trafficking of internalized virus-receptor complexes on infection; asking if this characteristic of an entry

${ }^{\ddagger}$ Adapted with permission. Krueger, R.L. and L. M. Albritton. Characteristics of the cellular receptor influence the intracellular fate and efficiency of retrovirus infection. $\mathrm{J}$ Virol, 2013. 87(10): p5916-5925. 
receptor influences infection. Subtypes SSTR-2, -3 and -5 were selected for this study because they are similar structurally and pharmacologically (e.g., kinetics of agonist binding and internalization) but have documented differences in intracellular trafficking that result in distinct intracellular fates after internalization (reviewed in [34]). SSTR-1 and -4 were not included because they are not as efficiently internalized as the other subtypes after agonist exposure [30]. I quantified Sst-RBS pseudovirion infection on host 293 cell lines stably expressing cell surface SSTR-2, -3 and -5 at levels comparable to each other, and to endogenous mCAT-1 expression on murine NIH 3T3 cells. This new model system circumvents the major technical constraints that have limited previous studies because the types of endocytic pathways and vesicular trafficking proteins available during infection are equivalent due to their 293 background. Additionally, the density of receptors and capacity for virus internalization are also comparable amongst the cell lines which were exposed to the same pseudovirus. Thus, differences in intracellular trafficking of internalized virus-receptor complexes should constitute the predominant influence on infection. Transduction assays revealed that Sst-RBS MLV and LV pseudotype transduction of the SSTR-2 and -3 cell lines was substantially lower than in the SSTR-5 expressing cells. Taken together the results of virus binding, internalization rate, lysosomatropic agent, protease inhibitor and penetration assays using quantitative real-time PCR support that infection via SSTR-5 is greater because viruses internalized by this subtype are directed to more permissive intracellular compartments, specifically to ones that are more favorable to penetration of the viral nucleocapsid than those compartments to which viruses bound to subtypes 2 and 3 are directed. These studies suggest that receptor characteristics such as intracellular trafficking exert a strong influence on the efficiency of infection and may have provided a selective pressure during retrovirus evolution guiding the choice of a host cell receptor.

\section{Results}

\section{Infection of Sst-RBS Pseudotyped Viruses Is Greatest via SSTR-5}

Chapter 3 described the generation and characterization of a population of 293 cells stably expressing cell surface SSTR-5 at levels comparable to the relative number of endogenous ecotropic receptors (mCAT-1) on murine NIH 3T3 cells (Figure 3-2). For these studies, two additional stable cell lines were sorted for levels of SSTR-2 and -3 comparable to that of the previously established 293/SSTR-5 cells (Figure 4-1) using an HA epitope tag present on each receptor subtype's extracellular amino-terminus. Fusion of the HA tag sequence in this position does not alter agonist binding or internalization [35]. These matched cell populations were exposed to equal volumes of serially diluted Sst-RBS, WT MLV or VSV G MLV and the level of LacZ transduction assessed by end point dilution titration. Surprisingly, transduction was 222-fold and 23-fold lower in 293/SSTR-2 and -3 cells, respectively, compared to the 293/SSTR-5 cells (Figure 4-2A). Infection of control VSV G MLV and LV pseudovirions was comparable in each of the cell types indicating that the observed infectivity differences were Sst-RBS- and receptor subtype-dependent and thus not due to unspecific differences between the cell lines. There was no observable infection of the parent 293 cells. The SST-14 ligand is capable of binding endogenous murine somatostatin receptors [27] and a low level of infection $(7.5 \pm 1.4 \mathrm{TU} / \mathrm{ml})$ was observed in NIH 3T3 cells exposed to Sst-RBS MLV. 

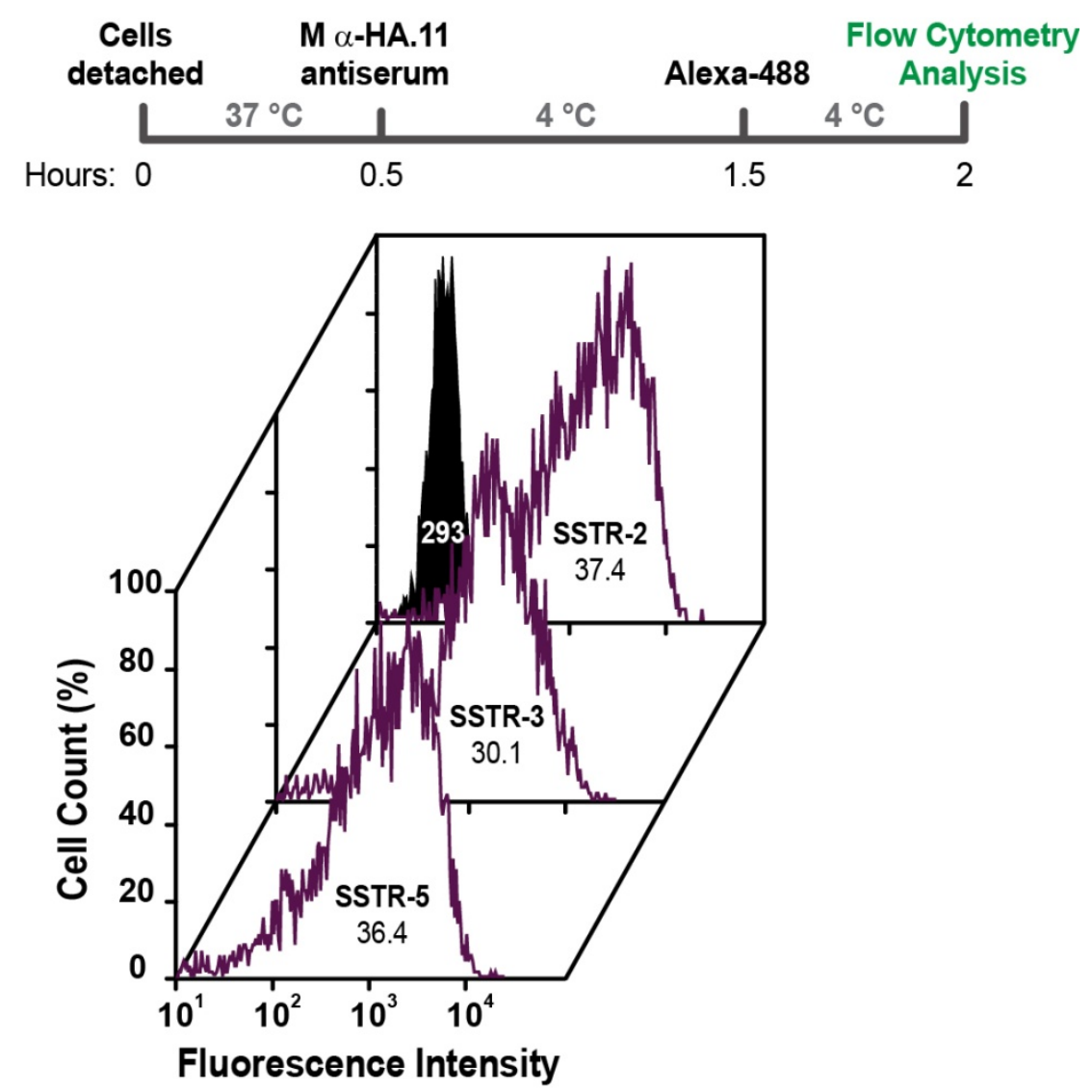

Figure 4-1. SSTR-expressing cell populations display comparable levels of target receptors on their cell surface

The steady state level of each SSTR subtype was quantified by flow cytometry using monoclonal antibody to the HA epitope tag located on the extracellular, amino-terminus of each receptor subtype. Representative histograms (purple, 293 cells expressing SSTR subtypes; black, background binding of the antibody to control 293 cells) are shown with values indicating the fold increase in mean fluorescent intensity over control 293 cells. 
A

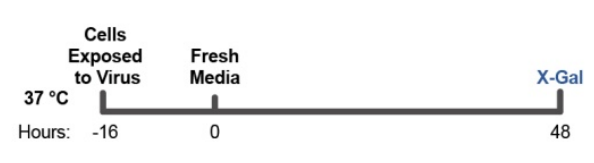

B

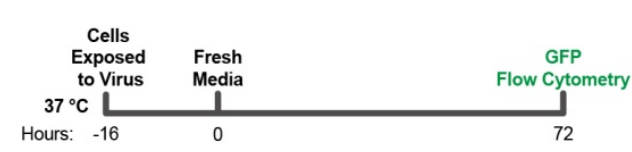

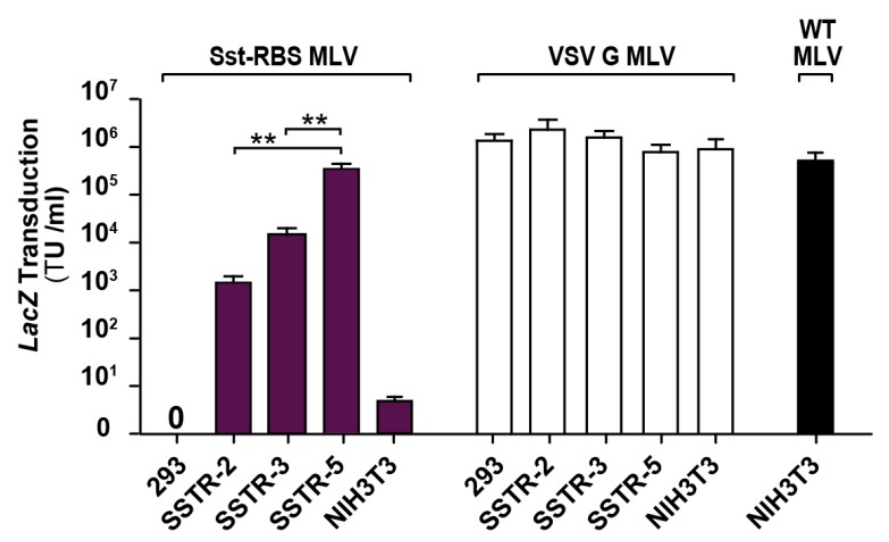

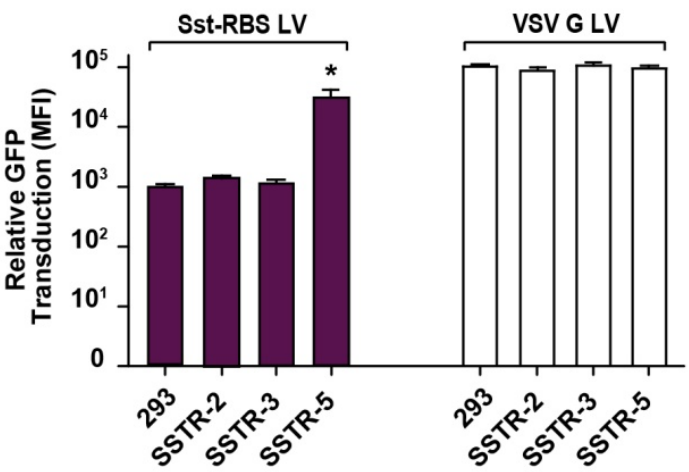

Figure 4-2. Infection of pseudotyped viruses is greatest via SSTR-5

A. 293 cells or the receptor matched 293/SSTR-2, -3 , or -5 populations were exposed to ten-fold serial dilutions of Sst-RBS (purple bars), VSV G (white bars) or WT (black bars) pseudotyped MLV, spinoculated and incubated at $37^{\circ} \mathrm{C}$ overnight. The level of infection was determined after 48 hours by scoring LacZ positive cells under a light microscope.

B. Sst-RBS [5X] or VSV G $\left(10^{-2}\right)$ pseudotyped LV was exposed to each of the indicated cell types, spinoculated and incubated at $37^{\circ} \mathrm{C}$ overnight. The level of infection was determined after 72 hours by flow cytometry analysis of GFP positive cells.

Values represent the mean $\pm S D$ of 3 independent titrations $(n=4)$. P-values were calculated by one way ANOVA and Bonferroni posttest. * $p \leq 0.05 ;{ }^{* *}, p \leq 0.01$. 
The trend observed with the MLV pseudovirions was also observed in five independent lentivirus infection assays using two pseudovirion stocks. Sst-RBS and VSV G LV particles transducing GFP were produced and the infectivity in 293, 293/SSTR-2, -3 , and -5 cells was analyzed by flow cytometry at $72 \mathrm{hpi}$. Sst-RBS mediated transduction was greater in SSTR-5 expressing cells with a mean of $0.9 \pm 0.5$ percent of cells expressing green fluorescence above the background levels in 293 cells (Figure 4-2B). In each of the three experiments a few GFP positive foci were observed in the 293/SSTR-2 and -3 populations and no GFP expression was observed in 293 cells by visual inspection using fluorescent microscopy prior to detachment for flow cytometry. However, the difference between the actual MFI of the SSTR-2 and -3 cells $\left(8.4 \times 10^{2}\right.$ and $7.4 \times 10^{2}$, respectively) and the background MFI of the 293 cells $\left(6.2 \times 10^{2}\right)$ was not statistically significant indicating that the low level of infection seen by visual inspection was below the level of detection by flow cytometry, evidently because the transduced cells represented an extremely low percentage of total cells. In two additional experiments, end-point dilution titration of LV transducing a puromycin resistance gene supported the flow cytometry results. Sst-RBS LV titers averaged $1 \times 10^{4} \mathrm{TU} / \mathrm{ml}$ in 293/SSTR-5 cells and zero in 293/SSTR-2 and -3 cells (no puromycin resistant colonies observed). VSV G LV displayed titers of greater than $1 \times 10^{5} \mathrm{TU} / \mathrm{ml}$ in all cell lines. The lower infection of Sst-RBS LV compared to VSV G pseudotypes was consistent with previous results; when pseudotyped on MLV, the entry capacity of Sst-RBS showed a mean of 2.7 which ranged up to 5-fold of VSV G (Figure 3-1) but was 200-fold less effective on LV pseudovirions as measured by luciferase transduction. In accordance with the GFP transduction results, a p24 ELISA of a parallel production of Sst-RBS and VSV G LV pseudovirions used in the flow cytometry analysis revealed a 2.3 fold difference in the number of physical particles (data not shown).

\section{Receptor Specific Interactions Mediate Sst-RBS MLV Infection}

Sst-RBS MLV infection of SSTR-2 expressing cells was previously shown to be specific to this subtype as judged by dose-dependent inhibition by exogenous SST-14 peptide [25]. To confirm that receptor specificity is retained by SSTR-3 and -5, end-point dilution titration was performed in the presence and absence of $1 \mu \mathrm{M}$ of the SST-14 peptide (Figure 4-3A). Sst-RBS MLV was competitively inhibited in each of the SSTRexpressing cells by more than $80 \%$ (Figure 4-3B). Infection of NIH 3 T3 cells by WT MLV or the SSTR expressing cells by VSV G MLV was unaffected by the presence of SST-14 (Figure 4-3C).The downstream effects of ligand-somatostatin receptor binding, signal transduction events and receptor desensitization are complex and can vary depending on the ligand and cell type [29, 30, 34, 36]. One important potential outcome involves the initiation of anti-proliferative signals within the cell [29] which is especially relevant to the life cycle of MLV because proviral integration into the host chromosome is highly dependent on the mitotic stage of cell division [9]. To determine if SSTR engagement affects the cell's ability to divide, the number of cells in each VSV G MLV positive foci (a cluster of $\beta$-galactosidase positive cells resulting from the division of one infected cell) was enumerated in the SST-14 treated and untreated populations from Figure 4-3C. If anti-proliferative signals are initiated through the SSTRs then the number of cells in each positive foci will be less in the SST-14 treated population than in the untreated cells. However, the mean number of cell divisions did not vary significantly among any of the SSTR cell types (Figure 4-4) indicating that the observed inhibition of infection via SSTR-2 and-3 was not due to a reduction in host cell division. 
A

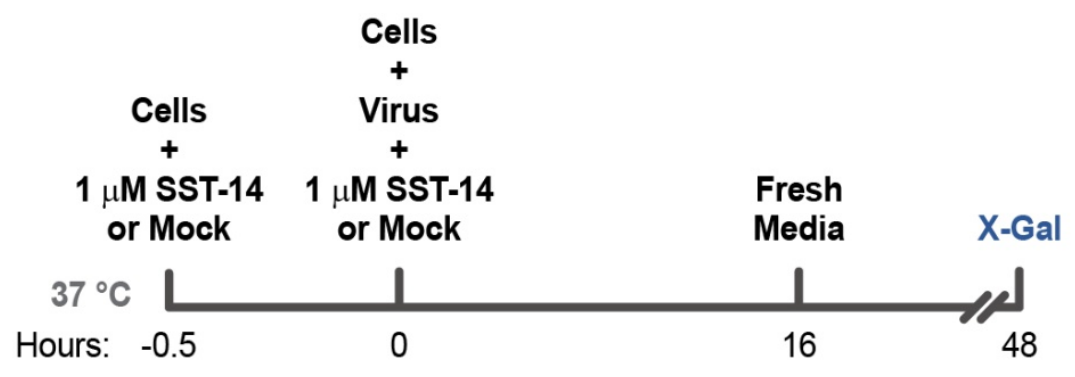

B

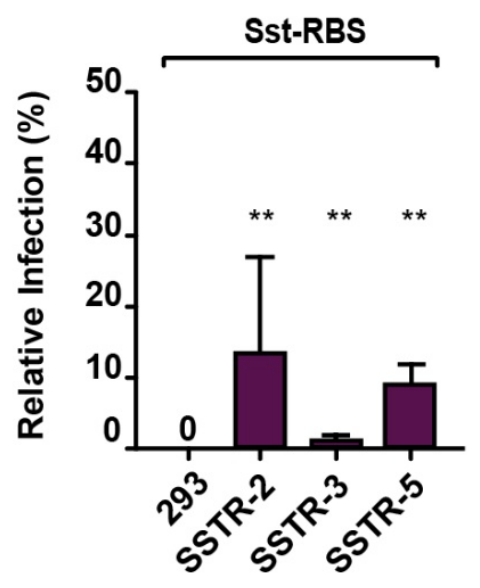

C

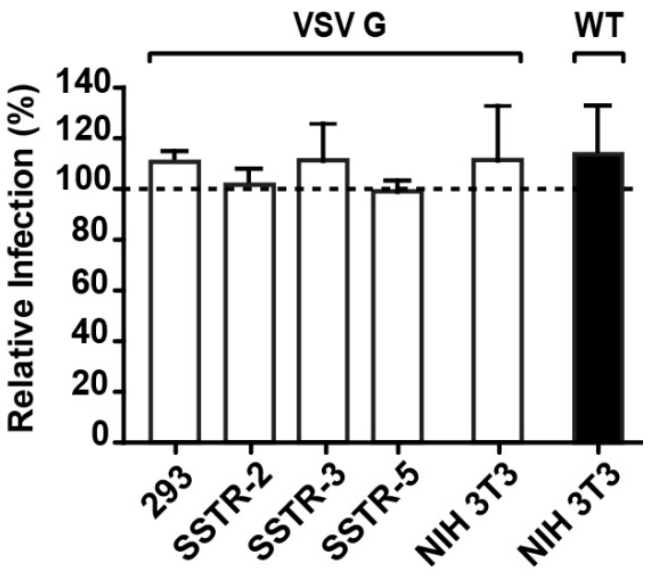

Figure 4-3. Receptor-specific interactions mediate Sst-RBS MLV infection

A. Experimental time line for transduction in the presence of SST-14 inhibitor.

B. Sst-RBS MLV infection was inhibited by preabsorption of cells with the SSTR agonist, SST-14. 293, 293/SSTR-2, -3, and -5 cells were incubated with $1 \mu \mathrm{M} \mathrm{SST-14}$ peptide or mock-treated for 30 minutes prior to spinoculation of Sst-RBS MLV (purple bars) in the presence of $1 \mu \mathrm{M}$ SST-14 or mock treated. Values show the mean relative infection \pm SD in the presence of the peptide, calculated as (infection in the presence of SST-14 / infection in its absence) $\times 100$.

C. The peptide agonist did not affect infection of control pseudovirions. Cells were treated as in (A) except that infection was performed with VSV G (white bars) or WT (black bars) MLV pseudovirion stocks.

All values represent the mean $\pm S D$ of three independent experiments $(n=4)$. $P$ values were calculated using two way ANOVA and Bonferroni post test. ${ }^{*}, p \leq 0.05 ;{ }^{* *}, p \leq 0.01$. 

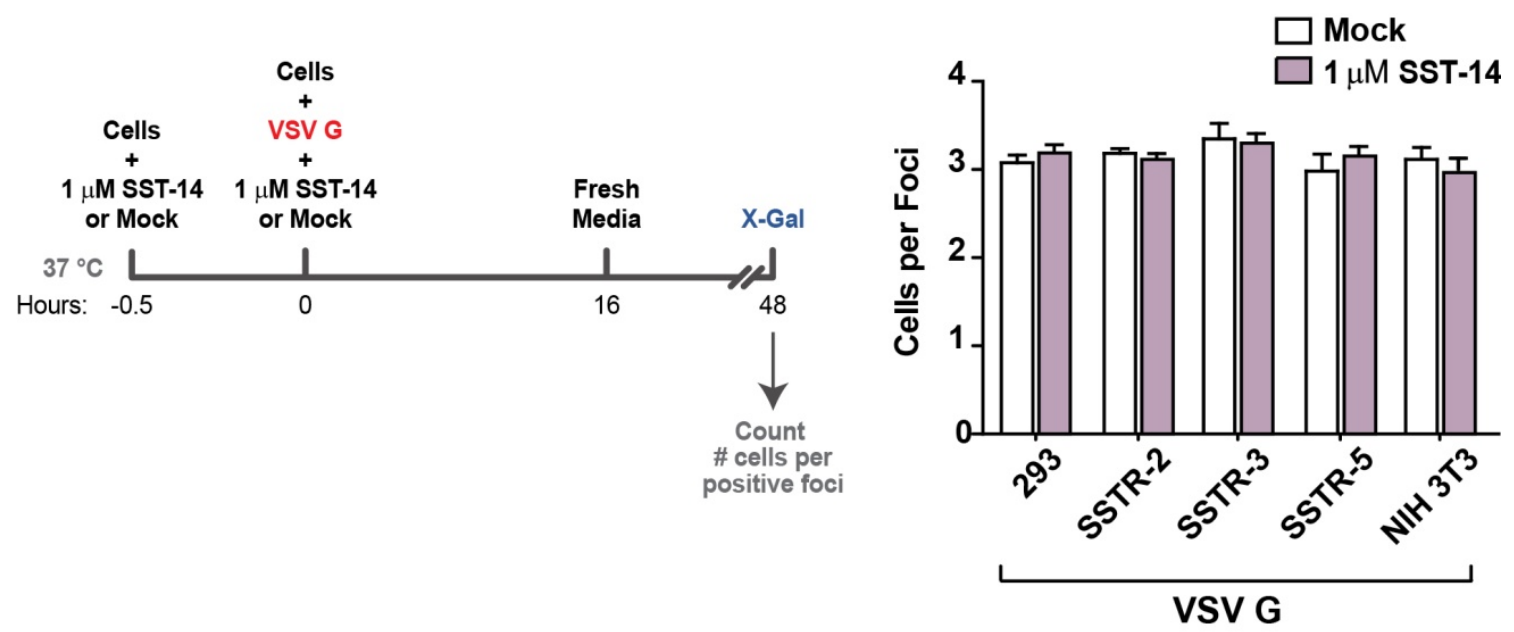

Figure 4-4. The SST-14 ligand does not act by inhibiting cell division

The mean number of cell divisions was determined by counting the number of cells in each $\beta$-galactosidase-positive foci from the VSV G MLV data in Figure 4-3B.

All values represent the mean $\pm S D$ of three independent experiments $(n=4)$. P values were calculated using two way ANOVA and Bonferroni posttest. ${ }^{*}, p \leq 0.05 ;{ }^{* *}, p \leq 0.01$. 


\section{The Relative Level of Cell-associated Virus Can Not Account for Differences in Transduction}

Although SSTR-2, -3 and -5 reportedly bind SST-14 with similar affinity [29], binding affinities between these somatostatin receptors and other ligands vary. For instance, while SSTR-2 and SSTR-3 bind the SST-28 precursor with comparable affinity to SST-14, SSTR-5 has a higher affinity for SST-28 [27, 30]. Furthermore, Octreotide, a synthetic peptide that mimics the agonist effects of the natural SST ligand, binds SSTR-2 with a higher affinity than both SSTR-3 and SSTR-5 [27, 34]. If binding to SSTR-2 and -3 is compromised by placing SST-14 in the larger context of the MLV Env, then increased infection of 293/SSTR-5 cells would be expected as the result of greater pseudovirion attachment. To investigate this possibility, equilibrium virus binding assays were performed as previously described $[42,70]$. Briefly, each of the SSTR-expressing cell lines were incubated with Sst-RBS MLV pseudovirions and the relative levels of attachment were quantified by flow cytometry using anti-SU antiserum. In three independent assays, Sst-RBS MLV showed a mean increase of $2.89 \pm 0.7$-fold in binding to 293/SSTR-5 over cells incubated with antibodies alone; an increase that was not significantly different from the mean for SSTR-2 and -3 cells $(2.27 \pm 0.6$ and $2.54 \pm 0.3$-fold, respectively). The histograms from a representative assay are shown in Figure 4-5A. However, Sst-RBS binding to 293 cells was also similar indicating that a majority of detectable virus binding can be attributed to a cellular factor common to 293 cells. An additional limitation of these results is that virus-receptor complexes internalized during the $37^{\circ} \mathrm{C}$ binding step would not be accounted for in this quantification of surface pseudovirions. Additional experiments in which the binding step was carried out at $4{ }^{\circ} \mathrm{C}$ to inhibit virus-receptor complex internalization yielded only background levels of Sst-RBS and WT MLV binding (data not shown), findings consistent with previous reports that MLV binding at lower temperatures is limited $[70$, 71].

To address the extent of virus-receptor complex internalization and control for virus associated with the SSTR-expressing cell lines due to their 293 cell parentage, a quantitative real-time PCR assay was designed to take advantage of the unique characteristic of many retroviruses, including MLV, wherein reverse transcription of genomic RNA (gRNA) has been primed and the Minus Strand Strong Stop (MSS) cDNA generated and packaged into mature virions along with the gRNA [72]. Using a primer set designed to specifically amplify the MSS species (Figure 4-5B); the relative level of receptor-associated virus particles (i.e. the amount of virus both internalized and bound at the cell surface) was measured as a more precise quantification of total virus. Internalization of virus was synchronized in each of the SSTR-expressing cell lines by spinoculation at $4{ }^{\circ} \mathrm{C}$ prior to shifting cells to $37^{\circ} \mathrm{C}$. At six hpi the amount of MSS cDNA was quantified by quantitative real-time PCR. There were no statistically significant differences in the fold increase of virus associated with each of the SSTR subtypes (Figure 4-5C). The amount of virus associated with each of the SSTR-expressing cell lines was also comparable at zero hpi (no $37^{\circ} \mathrm{C}$ shift) with mean relative fold increases of $1.1,1.1$ and 0.86 while the mean increases after 6 hours were $0.93,1.3$, and 0.99 for SSTR-2, SSTR-3 and SSTR-5; respectively. The difference between the amounts of cell associated virus at zero and $6 \mathrm{hpi}$ in each cell line was not significantly different indicating that the MSS signal was not amplified during the 6 hour incubation and that the MSS cDNA was synthesized in the mature virion prior to the start of the experiment. Together these experiments indicated that there are no differences in virus binding or, 
Figure 4-5. The relative level of cell-associated virus is comparable for each SSTR-expressing cell line

A. Equilibrium virus binding assay was performed using Sst-RBS MLV incubated with 293 or SSTR-expressing cells (binding to 293 cells is unpublished data). Antiserum to the MLV glycoprotein was used to detect surface-bound virus by flow cytometry analysis. Representative histograms and values indicating the fold increase in MFI \pm SD from three independent experiments are shown with surface-bound virus (purple) next to their mock-exposed counterparts (gray).

B. Schematic showing the location of quantitative real-time PCR amplicons within the recombinant MLV genome used in infection experiments. U3, unique 3' untranslated region, $R$, repeat sequence. U5, unique 5' untranslated region. LacZ, $\beta$-galactosidase gene. SV40, simian virus 40 promoter. Neo $r$, neomycin resistance gene.

C. Cells were exposed to Sst-RBS MLV or mock medium and spinoculated at $4{ }^{\circ} \mathrm{C}$ then shifted to $37^{\circ} \mathrm{C}$ to allow internalization. After six hours unbound virus was removed and cells were lysed and isolated DNA was used directly as the template for quantitative real-time PCR. The level of cell-associated virus in the SSTR-expressing cells was determined as the relative fold increase of the MSS amplicon for each SSTR expressing cell line over the 293 signal (representing background binding) normalized to endogenous $\beta$-actin. No amplification was detected in mock-exposed cells. Data represent three independent experiments $(n=3)$. Significance was calculated using one way ANOVA and Bonferroni posttest $P$ value $>0.5$. 
A
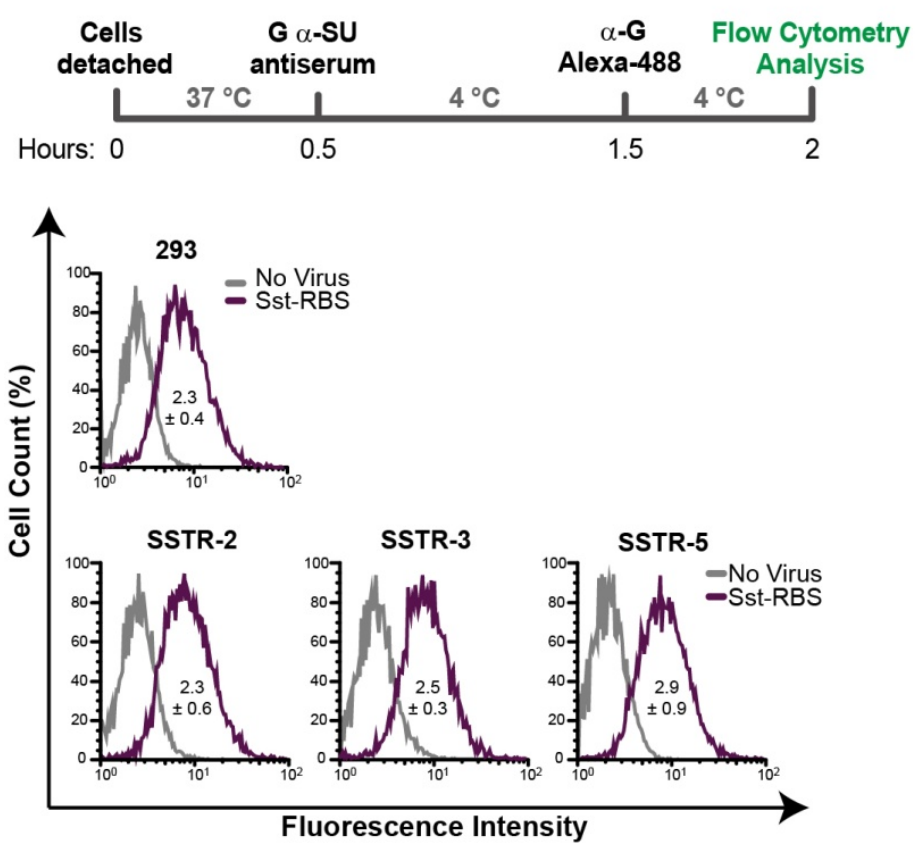

B

Total amount of

cell-associated virus

MSS cDNA amplicon

\begin{tabular}{|c|c|c|c|c|c|c|c|}
\hline U3 & R & U5 & LBS \\
\cline { 1 - 4 } & LacZ & SV40 & Neor & U3 & R & U5 \\
\hline
\end{tabular}

LacZ gene amplicon

Total amount of virus successfully

penetrated into cell cytoplasm

C
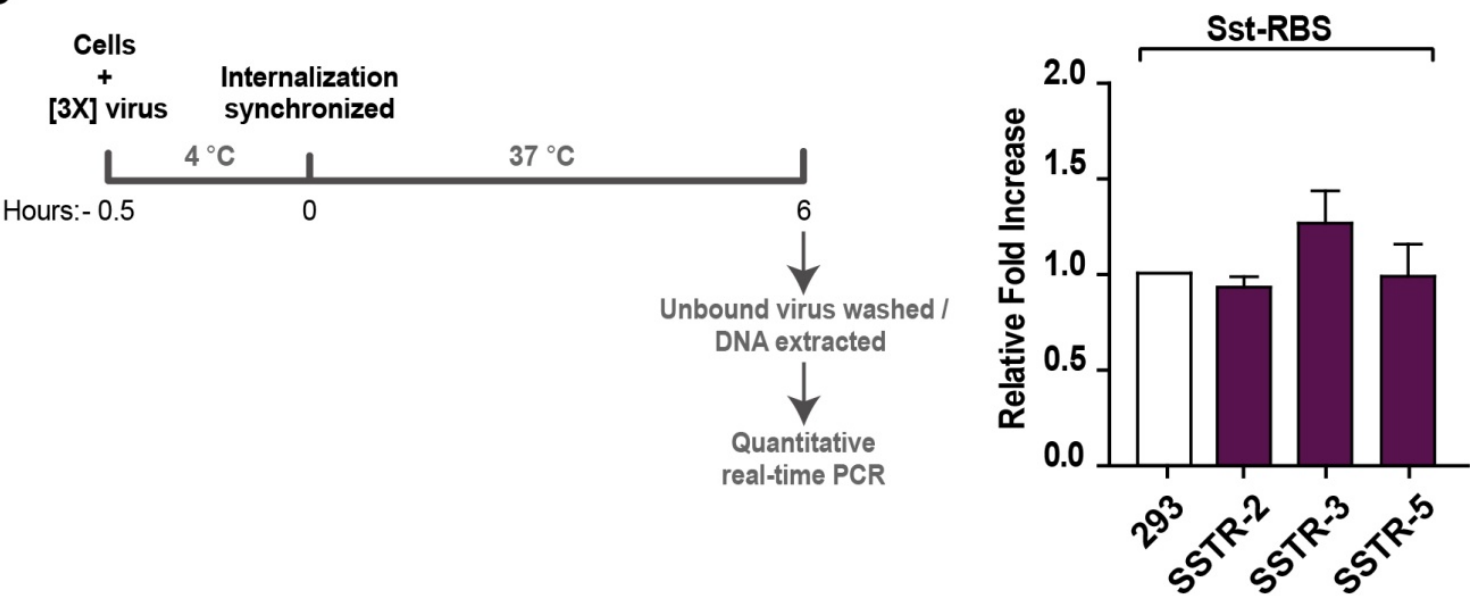
importantly, in the amount of cell-associated virus that can account for the higher level of infection via SSTR-5.

\section{Infection of Sst-RBS Pseudovirions Requires Internalization}

Additional experiments were performed to investigate the nature of the differences in infection of the three receptor subtypes. WT MLV is internalized by caveolinassociated endocytosis when bound to its natural receptor on NIH 3T3 cells [5], while the SSTR utilize clathrin-mediated endocytosis following SST-14 binding [30, 34]. To begin examining the possibility that internalization of Sst-RBS MLV bound to the three somatostatin receptor subtypes differs, infection was quantified in the presence of inhibitors of endocytosis (Figure 4-6A). Our lab [7, 43] and others [67, 73-75] have published the use of these inhibitors within the ranges indicated in this study with no detectable toxicity. To facilitate comparison to these previous publications, experiments were designed to use at least one "low" and "high" concentration within this range. Nocodazole, an inhibitor of microtubule polymerization that interferes with endocytic pathways used during virus entry. Nocodazole induced similar decreases in infection in all cell lines (Figure 4-6B), indicating that both WT and Sst-RBS MLV require endocytosis and, consequently, that the pseudovirions are not fusing at the cell surface.

Infection was also carried out in host cells pre-incubated with methyl- $\beta$-cyclodextrin $(\mathrm{M} \beta C D)$ which sequesters cholesterol and disrupts lipid rafts. Cholesterol-dependent endocytic pathways are strongly inhibited by this drug. Interestingly, infection via SSTR-5 exhibited a mean inhibition of $43.0 \%$ to the lowest concentration of the drug that was similar to the level of sensitivity of control WT MLV on mCAT-1 in NIH 3T3 cells (mean of $37.9 \%$ inhibition). Infection of $293 /$ SSTR-2 and -3 cells was also sensitive to $\mathrm{M} \beta C D$ (means of 18.3 and $24.4 \%$ inhibition, respectively) although the lower mean inhibitions were not statistically different from the means for SSTR-5 or NIH 3T3 (Figure 4-6C). While these results confirm that internalization is required for entry via all three SSTR subtypes, it is also noteworthy that entry of Sst-RBS MLV through SSTR-5 showed a cholesterol-dependence that was more similar to WT MLV on NIH 3T3 cells than to entry via the other two SSTR subtypes.

\section{A Slower Rate of Internalization Correlates with Enhanced Infection}

The efficiency of receptor internalization was evaluated by incubating the SSTR subtype expressing cell lines with $1 \mu \mathrm{M}$ SST-14 or mock treatment for 30 minutes at $4{ }^{\circ} \mathrm{C}$ or $37^{\circ} \mathrm{C}$ to stimulate receptor internalization. Cells were then incubated for 1 hour at $4{ }^{\circ} \mathrm{C}$ with mouse anti-HA.11 monoclonal antibody (Figure 4-7A). A decreased MFI after treatment with $1 \mu \mathrm{M}$ SST-14 was viewed as evidence that SSTR internalization occurred. The amount of internalization of each of the receptor subtypes at $4{ }^{\circ} \mathrm{C}$ (at which temperature receptor internalization should be inhibited) was then compared to the amount of internalization at $37^{\circ} \mathrm{C}$. The profile of 293 cells (which do not express any HA-tagged SSTR) does not change while the peaks for SSTR-2 and -3 reveal receptor internalization at $37^{\circ} \mathrm{C}$, each representing a mean fold increase in internalization of 27

over that of control 293 cells. However, the peaks for SSTR-5 remain static, representing a mean fold increase in internalization of 8.8 (Figure 4-7B). This incongruence with 
A

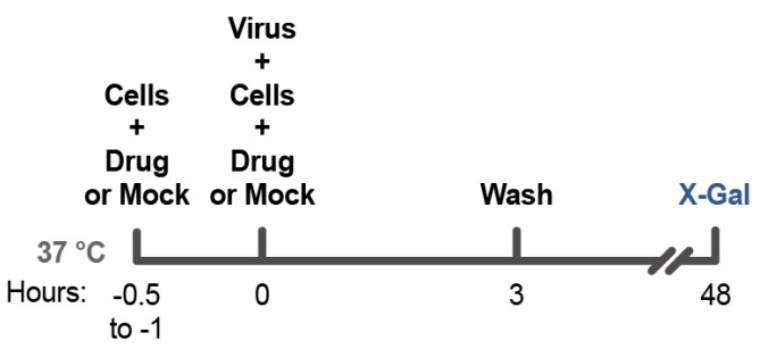

B

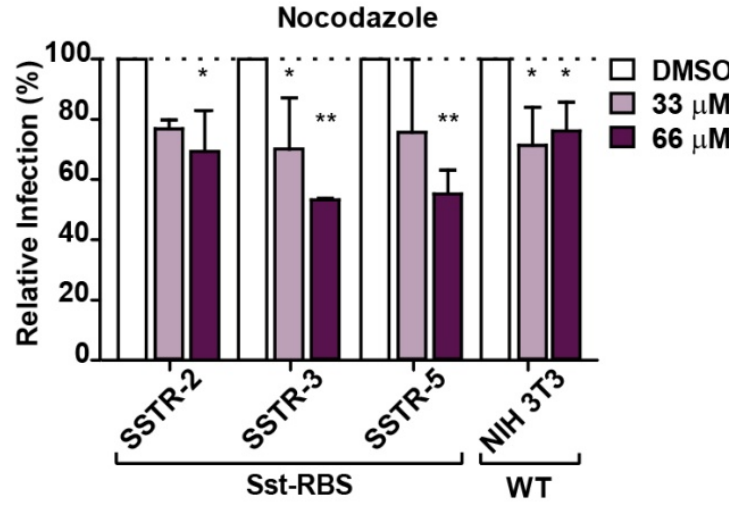

Inhibits microtubulin polymerization

C

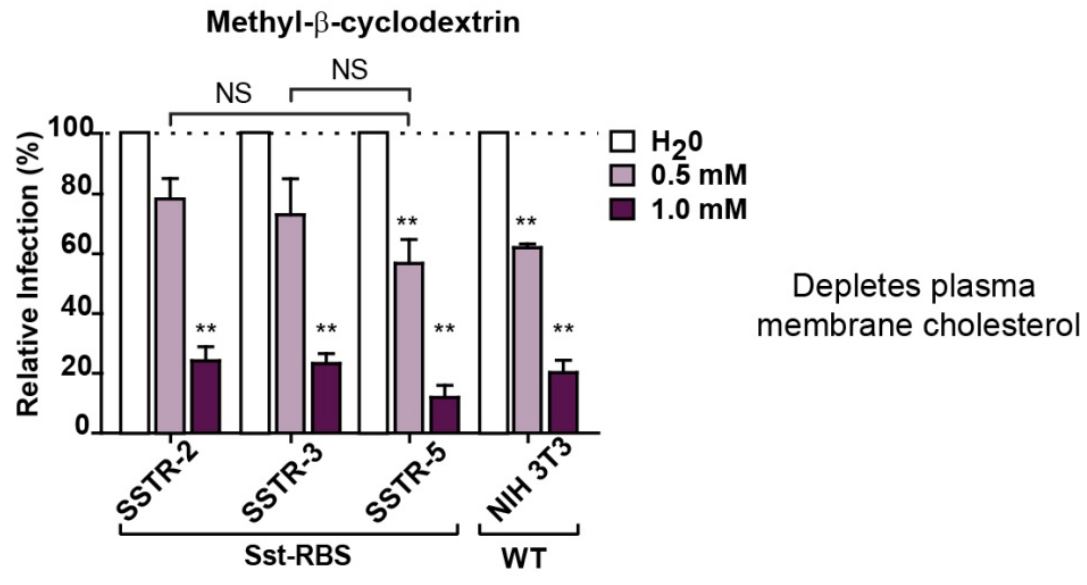

Figure 4-6. Infection of Sst-RBS pseudovirions requires internalization

A. Experimental time line for drug inhibitor studies.

B and C. 293, 293/SSTR-2,-3 and -5 or NIH 3 T3 cells were pretreated with (B) DMSO, $33 \mu \mathrm{M}$ or $66 \mu \mathrm{M}$ nocodazole for 1 hour or $(\mathrm{C}) \mathrm{H}_{2} \mathrm{O}, 1 \mathrm{mM}$ or $0.5 \mathrm{mM} \mathrm{M \beta CD}$ for 30 minutes then exposed to Sst-RBS or WT MLV maintaining drug or vehicle. Infection was scored with Romanizer software for the presence of $\beta$-galactosidase positive cells at 48 hpi. Relative infection was calculated as the percentage of infection compared to the vehicle only treated cells. No transduction was observed in 293 cells (data not shown).

Data represent the mean $\pm S D$ of three independent experiments $(n=4)$. $P$ values were calculated by using two way ANOVA and Bonferroni posttest. NS, Not Significant;

${ }^{*}, p \leq 0.05 ;{ }^{* *}, p \leq 0.01$. 
Figure 4-7. Patterns of receptor internalization among SSTR subtypes

A. Experimental time line for receptor internalization study.

B. Representative histograms indicating the level of cell surface receptor after 30 minutes in the absence or presence of $1 \mu \mathrm{M}$ SST-14 ligand at $4{ }^{\circ} \mathrm{C}$ or $37^{\circ} \mathrm{C}$ as determined by flow cytometry using the HA epitope tag located on the amino-terminus of each SSTR subtype (unpublished data). 293 cells do not express HA tagged SSTR. Decreased MFI of peaks at $37^{\circ} \mathrm{C}$ with $1 \mu \mathrm{M}$ SST-14 indicate internalization of surface receptors.

C. Relative internalization of HA-tagged SSTR in each cell line (unpublished data). The relative internalization was calculated by comparing the MFI of SST-14 treated cells at $4{ }^{\circ} \mathrm{C}$ to the MFI of SST-14 treated cells at $37^{\circ} \mathrm{C}$ and expressed as the mean fold increase in internalization, as indicated below the respective bar.

Data represent the mean $\pm S D$ of three independent experiments. Significance was calculated by using two way ANOVA and Bonferroni posttest $P$ value $>0.5$. 
A Cells Detached + I -

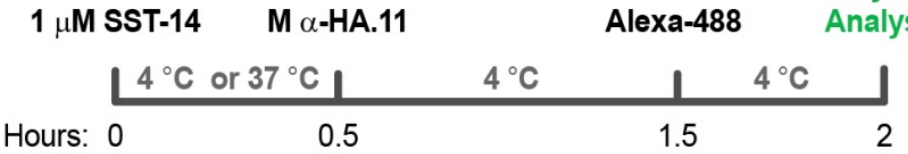

B

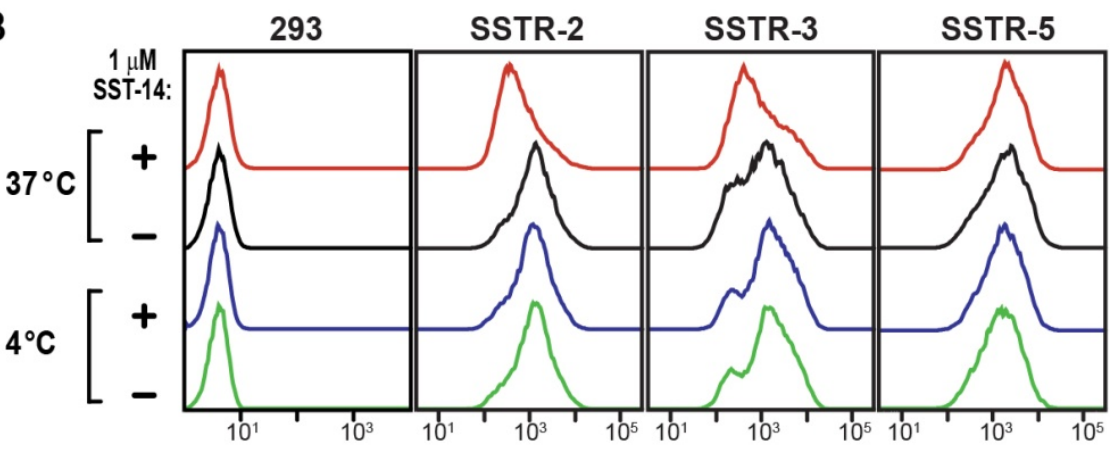

C

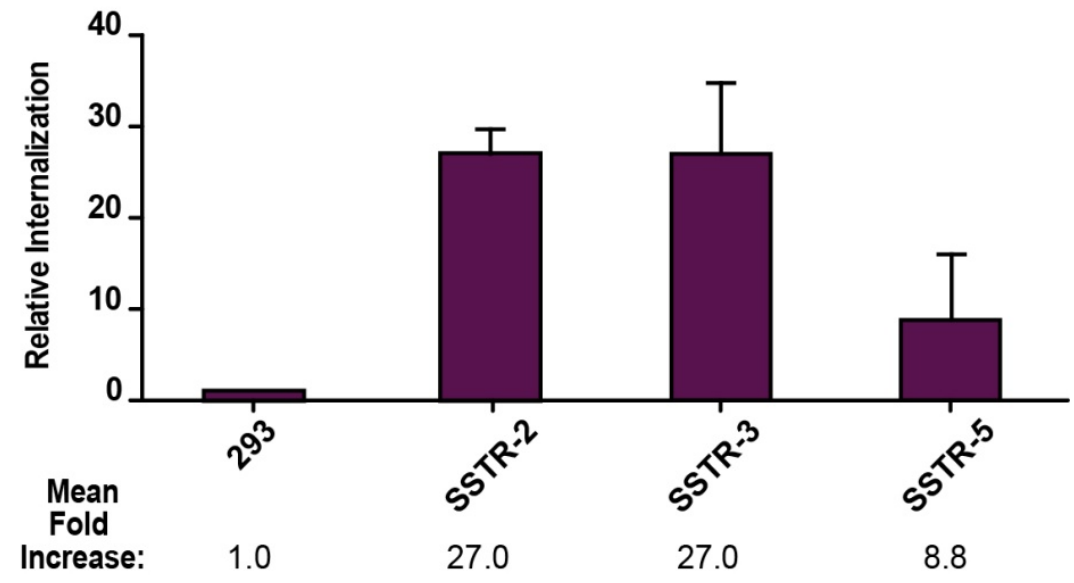


infection results lead to the hypothesis that SSTR-5 was internalized more slowly than SSTR-2 and -3 .

Thus, the kinetics of virus-receptor complex internalization were directly investigated by examining the level of infection over time and under conditions in which virus attachment was synchronized and non-internalized virus was inactivated at early time points. Attachment was synchronized by spinoculation at $4{ }^{\circ} \mathrm{C}$ and internalization of bound virus was initiated by shifting the cultures to $37^{\circ} \mathrm{C}$. At various times after the temperature shift, non-internalized virus was inactivated by a low-pH citrate wash and infection was allowed to continue (Figure 4-8A). The level of transduction at each time point was normalized to the values obtained at one hour; at which time infection had reached an average of $92.1 \%$ of the levels obtained from overnight exposure of the same virus stocks on 293/SSTR-5 (data not shown). The normalized levels of transduction were graphed and the slope of each curve calculated by non-linear regression analysis to determine the relative rates of internalization (percentage per minute) for each virus-receptor combination. Surprisingly, Sst-RBS MLV was internalized faster in 293/SSTR-2 and -3 cells (Figure 4-8B) as indicated by $50 \%$ of infectious virus $\left(T_{50}\right)$ having been internalized at 12.4 and 13.3 minutes, respectively, compared to 19.7 minutes in SSTR-5 expressing cells. Like the outcome of M $\beta C D$ treatment, the $T_{50}$ in SSTR-5 cells was similar to that of WT MLV in NIH 3T3 cells (17.7 minutes). No infection of either virus was seen in the parent 293 cells.

\section{SSTR-5 Directs Virus to a More Permissive Environment}

The differences in internalization presented in Figure 4-8 strongly suggest that entry through SSTR-5 may be directing Sst-RBS MLV to an intracellular environment that is more permissive for infection. To investigate this possibility, infection was examined in the presence of bafilomycin A1 (BafA) (a vacuolar ATPase inhibitor). This drug interferes with the acidification of endosomes and is commonly used to determine the $\mathrm{pH}$ requirements of the membrane fusion step during entry [55]. For example, WT MLV is not inhibited by BafA while VSV G mediated infection is profoundly decreased [73]. Unexpectedly, BafA enhanced, rather than reduced, infection through SSTR-2 (mean transduction increased from $2.9 \pm 1.6 \mathrm{TU} / \mathrm{ml}$ in DMSO treated cells to $7.5 \pm 4.1 \times 10^{1}$ $\mathrm{TU} / \mathrm{ml}$ in cells treated with $10 \mathrm{nM}$ BafA) and SSTR-3 (from $8.6 \pm 3.1$ to $22 \pm 6.4 \times 10^{1}$ $\mathrm{TU} / \mathrm{ml}$ ); whereas Sst-RBS MLV infection via SSTR-5 (from $2.6 \pm 0.6$ to $2.0 \pm 0.9 \times 10^{3}$ $\mathrm{TU} / \mathrm{ml}$ ) was not significantly affected by the drug (Figure 4-9A). While BafA enhanced infection via SSTR-2 and -3 , the total amount of transduction remained logs below that seen in 293/SSTR-5 cells or in WT-MLV infection of NIH 3T3 cells. These results are consistent with exposure to an acidic environment, such as that found in late endosomes, inhibiting Sst-RBS MLV infection through SSTR-2 and -3 but not through SSTR-5. Results of infection in the presence of chloroquine (Chlor) (a weak base that accumulates in lysosomal compartments) were uninformative in that similar inhibition was observed in all cell types (Figure 4-9B). However, monensin had a more marked effect on infection in 293/SSTR-2 than BafA. A member of the carboxylic ionophore class of drugs monensin functions as a sodium ion exchanger also raising the $\mathrm{pH}$ of lysosomal compartments [6, 55, 76]. Infection through SSTR-2 increased from $13.4 \pm 2.6$ to $124 \pm 59.4 \mathrm{TU} / \mathrm{ml}$ in the presence of monensin which represents a 9.9 fold increase while infection of 293/SSTR-5 was unaffected in its presence (Figure 4-9C). 


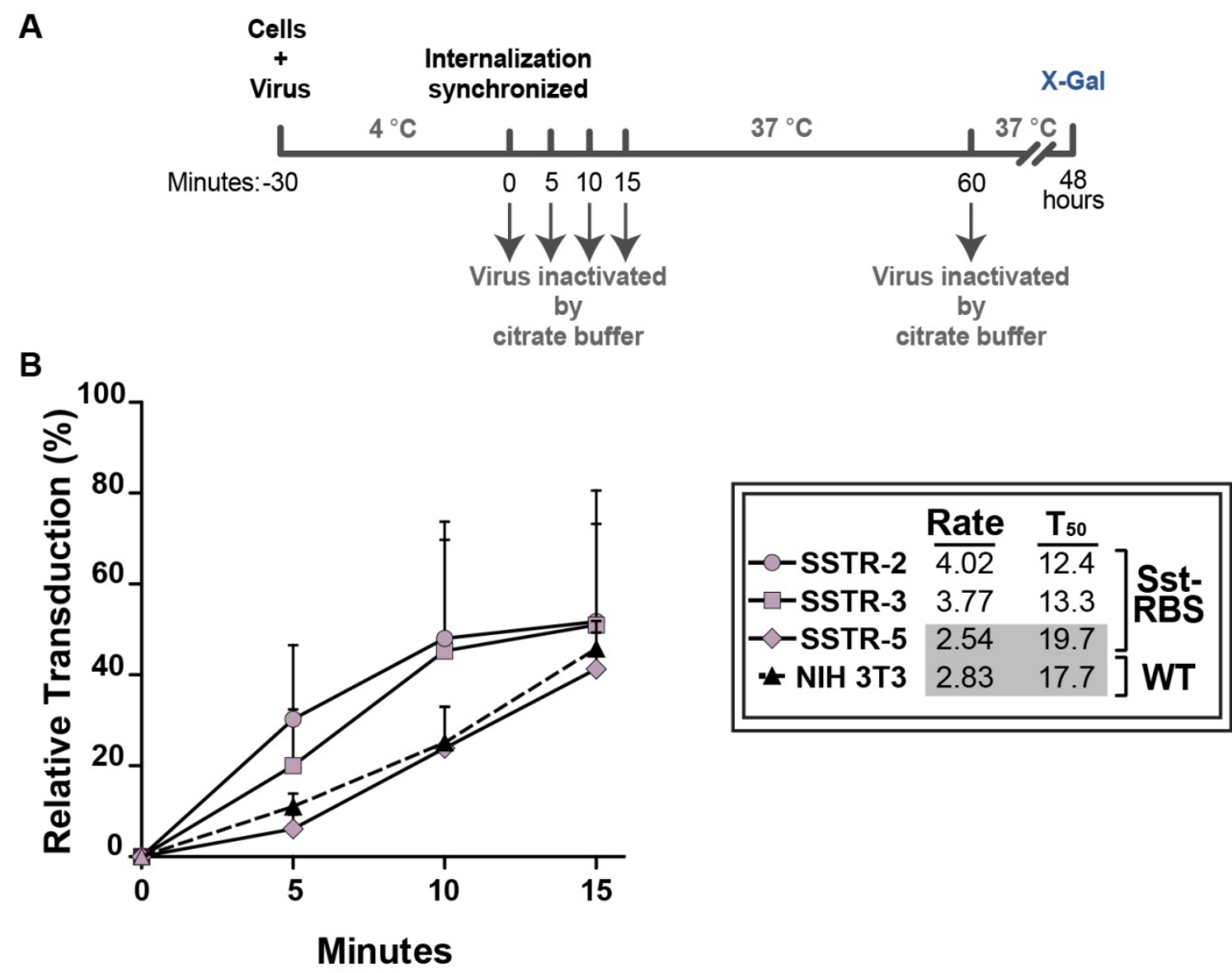

Figure 4-8. A slower rate of virion internalization correlates with increased infection

A. Experimental time line for rate of internalization study.

B. Sst-RBS or WT MLV was spinoculated onto 293, 293/SSTR-2, 3 and 5 or NIH 3T3 cells at $4{ }^{\circ} \mathrm{C}$ then shifted to $37^{\circ} \mathrm{C}$ for the indicated time to allow internalization. Noninternalized virus was inactivated and infection measured at $48 \mathrm{hpi}$ by counting LacZ positive cells using Romanizer software [44]. Values shown are the relative infection calculated as (infection at each time point / infection at one hour) $\times 100$. The relative rate of internalization (\% pseudovirions internalized per minute) was calculated as the slope of each curve using non-linear regression analysis (GraphPad Prism). $T_{50}$, time required to obtain $50 \%$ infection.

Data represent the mean $\pm S D$ of four independent experiments $(n=4)$. 
Figure 4-9. Neutralizing acidic compartments enhances infection via SSTR-2

\begin{abstract}
A, B and C. 293, 293/SSTR-2,-3 and -5 or NIH 3 T3 cells were pretreated with (A) DMSO, $10 \mathrm{nM}, 20 \mathrm{nM}$, or $30 \mathrm{nM}$ BafA (B) $\mathrm{H}_{2} \mathrm{O}, 15 \mu \mathrm{M}$ or $30 \mu \mathrm{M}$ Chlor or (C) EtOH, 12.5 $\mu \mathrm{M}$ or $25 \mu \mathrm{M}$ Monensin prior to Sst-RBS, VSV G or WT MLV exposure, as indicated, while maintaining the drug or vehicle. Infection was scored with Romanizer software for the presence of $\beta$-galactosidase positive cells at $48 \mathrm{hpi}$ Relative infection was calculated as the percentage $(A, B)$ or fold increase $(C)$ of infection compared to the vehicle only treated cells. There was no observable infection of 293 cells in the presence or absence of chloroquine, but increased to $14 \mathrm{TU} / \mathrm{ml}$ at $10 \mathrm{nM}$ BafA, and $14.77 \pm 5.3$ at $12.5 \mu \mathrm{M}$ and $19.07 \pm 4.3 \mathrm{TU} / \mathrm{ml}$ at $25 \mu \mathrm{M}$ Monensin.
\end{abstract}

All data represent the mean $\pm S D$ of at least three independent experiments $(n=4)$. $P$ values were calculated by using two way ANOVA and Bonferroni posttest. *, $p \leq 0.05$; ${ }^{*}$, $\mathrm{p} \leq 0.01$. 
A

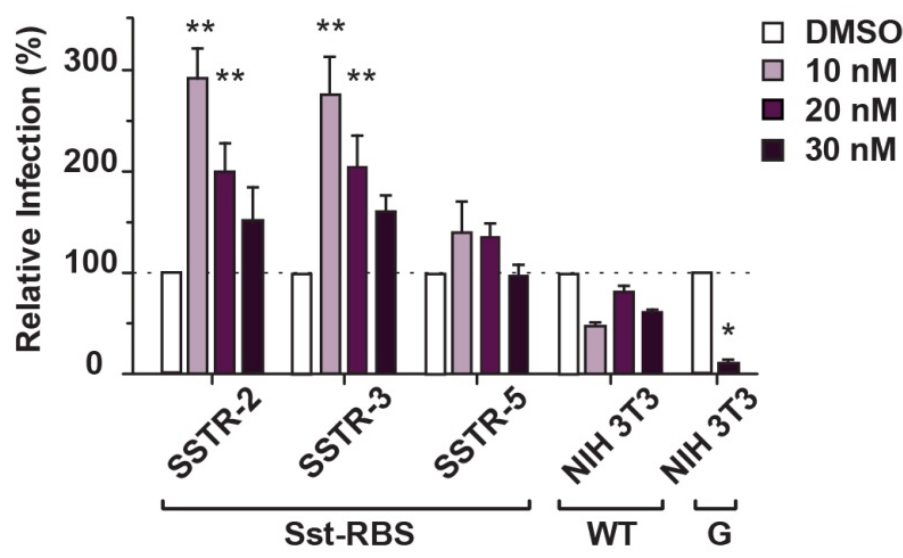

Vacuolar ATPase Inhibitor

Inhibits endosomal acidification and low pH enzymatic activity

B

Chloroquine

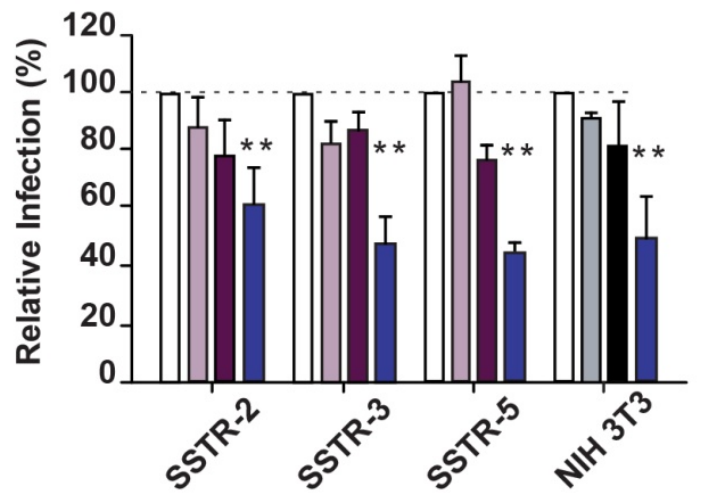

$\square \mathrm{H}_{2} \mathrm{O}$

$\square 15 \mu \mathrm{M}]$ Sst-

$\square 30 \mu \mathrm{M}]$ RBS

$\left.\begin{array}{l}15 \mu \mathrm{M} \\ \square 30 \mu \mathrm{M}\end{array}\right] \mathrm{WT}$

$\square 30 \mu \mathrm{M}-\mathrm{VSV} \mathrm{G}$

\section{Weak Base \\ Accumulates in lysosomes and inhibits acidification}

C

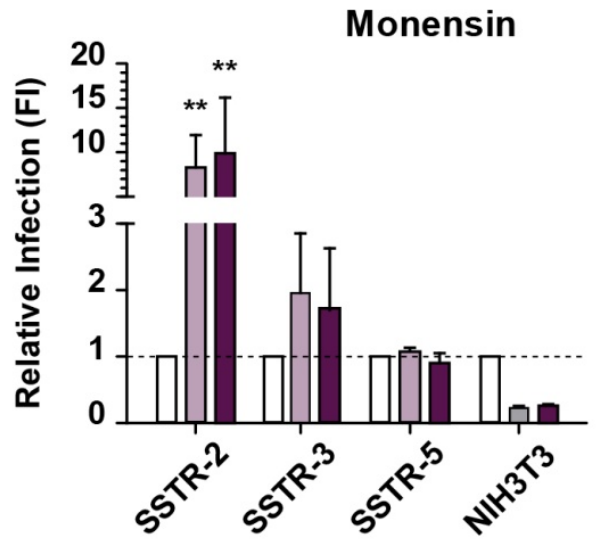

Sodium lon Exchanger Inhibits lysosomal acidification and low pH enzymatic activity 
Since host cell proteases of the cathepsin family have been shown to be important in potentiating infection of WT MLV by activating the fusion capacity of the viral glycoprotein [7, 74], it is possible that SSTR-2 and -3 transport virus to intracellular compartments containing either an unfavorable type or concentration of protease. To examine this possibility, infection was performed in the presence of CA074 Me. At low concentrations, CA074 Me specifically inhibits cathepsin B, while at higher concentrations it also inhibits other cysteine cathepsins [7]. Infection of Sst-RBS MLV was reduced at the higher, non-specific concentration but was not changed significantly at $5 \mu \mathrm{M}$ for all receptor subtypes, whereas; WT MLV infection of control NIH 3T3 cells was inhibited significantly at both concentrations (Figure 4-10A). These results suggest that, in a 293 cell background, cathepsin B is not the predominant protease involved in entry of Sst-RBS MLV. To further explore the influence of cellular proteases, infection was quantified in the presence of leupeptin, a lysosomal protease inhibitor. Sst-RBS MLV infection decreased markedly in 293/SSTR-2 and SSTR-3 while only modest decreases were seen in 293/SSTR-5 (Figure 4-10B), indicating that the protease involved in infection through SSTR-5 differs from those encountered by virus internalized via SSTR-2 and -3. Taken together, I favor the interpretation of these drug studies to indicate that SSTR-5 directs virus to a distinct intracellular compartment that is highly favorable for infection, at least in part because it contains a favorable set of cellular proteases for activation of the Sst-RBS glycoprotein.

\section{Penetration into the Host Cytoplasm Is More Efficient via SSTR-5}

If this interpretation is correct, then the greater infection via SSTR-5 could be explained by more efficient protease activation of virus-cell membrane fusion leading to greater levels of penetration. To test this hypothesis, the relative amount of virus that had penetrated the host cell cytoplasm was quantified. In MLV, reverse transcription is initiated during virion assembly but halts shortly after the MSS cDNA is generated. Then in a second stage, shown to occur after penetration, the cDNA of internal genes is synthesized [58, 72]. Based on this attribute, Gao and colleagues developed a quantitative real-time PCR assay using the amplification signal of an internal gene as a measure of virus penetration and infection [45]. We adapted this assay to compare penetration of MLV pseudovirions among the SSTR subtypes by measuring the level of internal LacZ gene amplification after 6 hours (Figure 4-11A). The schematic in Figure 4-5B shows the location of the amplicon within the internal LacZ gene, as well as the MSS cDNA amplicon. The LacZ amplification signal for each cell line was normalized to the MSS signal for the zero and 6 hour incubation times to account for bound but not internalized virions. Increased $L a c Z$ amplification then becomes proportional to the number of virion cores that penetrate the host cytoplasm. When the normalized LacZ amplification data were expressed as the relative fold increase from zero to $6 \mathrm{hpi}$, 293/SSTR-2 and -3 cells showed significantly lower levels of reverse transcription than 293/SSTR-5 cells and NIH 3T3 cells exposed to WT-MLV over the 6 hour time period (Figure 4-11B). These data indicate that a partial block to reverse transcription occurred after virus binding and internalization into 293/SSTR-2 and -3 cells, supporting the interpretation that the endocytic pathway utilized by SSTR-5 directs virus to a more permissive environment for penetration. 
A CA074 Me

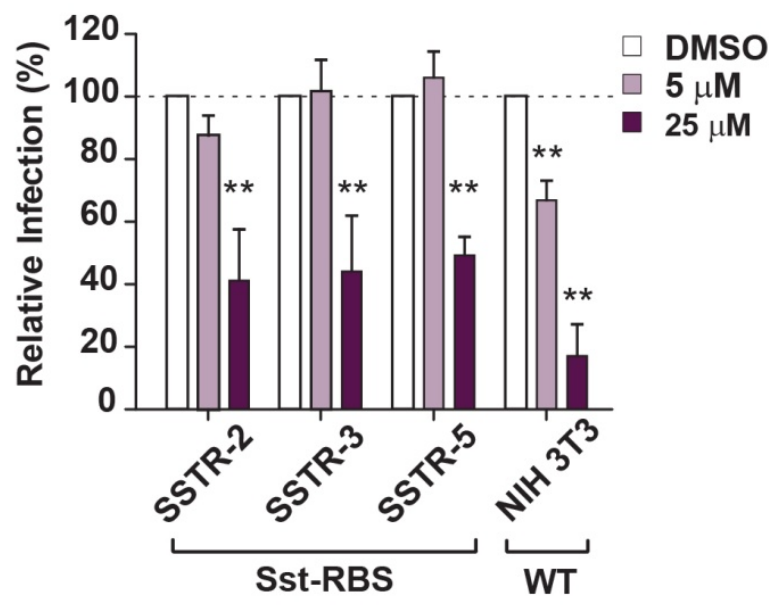

Cathepsin B inhibitor

Inhibits cathepsin B enzyme activity and other cysteine proteases at high concentrations

B Leupeptin

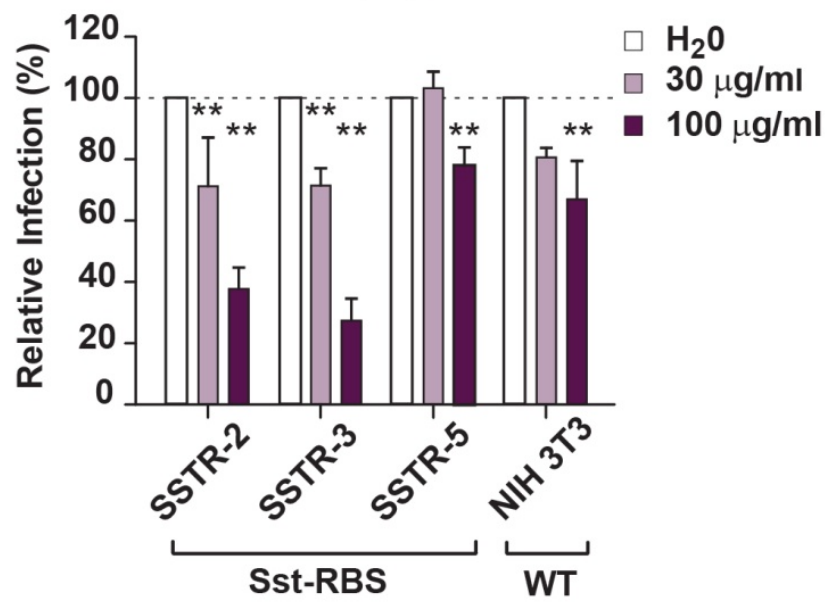

Lysosomal protease inhibitor

Figure 4-10. SSTR-5 internalizes to a more permissive intracellular environment

A and B. 293, 293/SSTR-2,-3 and -5 or NIH 3 T3 cells were pretreated with (A) DMSO, 5 $\mu \mathrm{M}$ or $25 \mu \mathrm{M}$ CA074 Me or (B) $\mathrm{H}_{2} \mathrm{O}, 30 \mu \mathrm{g} / \mathrm{ml}$ or $100 \mu \mathrm{g} / \mathrm{ml}$ leupeptin prior to Sst-RBS, VSV G or WT MLV exposure, as indicated, while maintaining the drug or vehicle. Infection was scored with Romanizer software for the presence of $\beta$-galactosidase positive cells at $48 \mathrm{hpi}$ Relative infection was calculated as the percentage of infection compared to the vehicle only treated cells. There was no observable infection of 293 cells in the presence or absence of CA074 Me or leupeptin.

All data represent the mean $\pm S D$ of at least three independent experiments $(n=4)$. $P$ values were calculated by using two way ANOVA and Bonferroni posttest. ${ }^{* *}, p \leq 0.01$. 


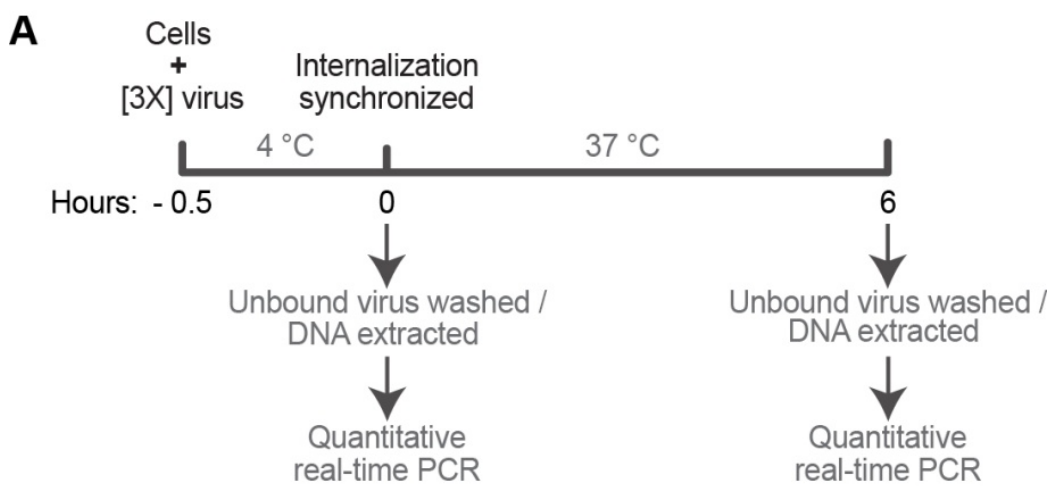

B

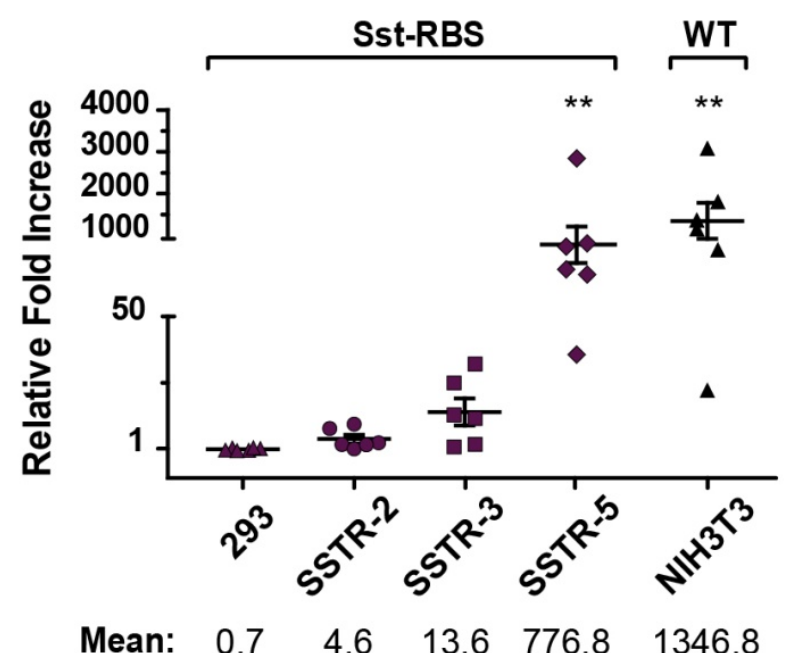

Figure 4-11. Virus internalized via SSTR-5 more efficiently penetrates into the host cytoplasm

A. Whole cell DNA was isolated from 293, SSTR-expressing and NIH 3 T3 cells exposed to Sst-RBS or WT MLV. DNA was isolated from cells incubated with virus at $4{ }^{\circ} \mathrm{C}$ but not shifted to $37^{\circ} \mathrm{C}$ and from replicate cell cultures 6 hours after a $37^{\circ} \mathrm{C}$ shift to allow internalization, membrane fusion and viral nucleocapsid penetration of the host cell cytoplasm as indicated in the experimental time line.

B. Results obtained from the MSS and LacZ amplicons were normalized to the $\beta$-actin signal for the respective host cell line and the fold increase in the level of reverse transcription was calculated as a measure of virion penetration by comparing LacZ gene amplification to the MSS amplification signal over the 6 hour time period. No amplification was detected in mock-infected cells.

Each symbol represents the relative fold increase in pseudovirion penetration from one independent experiment. The mean relative fold increase from six independent experiments is shown below the graph $(n=3)$. $P$ values were calculated using one way ANOVA and Dunn's posttest. ${ }^{* *}, p \leq 0.01$. 


\section{Discussion}

This chapter reports observable differences in the level of infection of Sst-RBS pseudotyped retroviruses when exposed to 293 cells stably expressing somatostatin receptor subtypes 2, 3 and 5 (Figures 4-2A and B) that correlate with distinctive receptor subtype-specific characteristics. When facilitated by SSTR-2 and -3 , infection is restricted at a point after receptor binding and internalization (Figures 4-5 and 4-6B) but prior to penetration into the host cytoplasm (Figure 4-11). In contrast, SSTR-5 mediated entry featured slower internalization with characteristics similar to that observed for WT MLV (Figures 4-7 and 4-8). Virus bound to SSTR-5 was directed to an intracellular environment that allowed near WT levels of penetration (Figure 4-11) possibly due to a more favorable complement of host cell proteases (Figure 4-10).

Taken together I interpret these results to support that the observed differences in infection between SSTR subtypes are primarily due to differences in the fate of internalized virus-receptor complexes. I favor that the intracellular trafficking patterns accessible to each subtype impose constraints on internalized virus-receptor complexes and direct viruses to more, or less, permissive environments. Figure 4-12 shows a model of virus entry illustrating these observations and the proposed pathways used by the SSTR subtypes. This interpretation is consistent with reports of agonist-induced SSTR desensitization (natural ligand removal and receptor recycling or degradation). While SSTR-2, -3 and -5 are similar in structure, kinetics of agonist binding and internalization [30], the subtypes differ in their intracellular trafficking during agonist-induced desensitization. SSTR-5 lacks the clusters of Ser/Thr phosphate acceptor sites found on the cytoplasmic tails of subtypes 2 and 3 [30, 34, 77]. These clustered phosphate acceptor sites have been shown to control the intracellular destination of ligand-SSTR complexes by regulating $\beta$-arrestin and scaffolding protein recruitment to endocytic vesicles resulting in distinct intracellular fates for the internalized ligand-receptor subtype complexes [30, 36, 77].

Inhibition during virus binding and internalization would represent relatively trivial explanations for the different levels of infection observed among the somatostatin subtype expressing cell lines; whereas effects on fusion and penetration are of greater interest. A standard virus binding assay was unable to detect Sst-RBS MLV binding to any of the SSTR subtypes that was above the level of binding to control 293 cells, and the relative amount of cell-associated virus was similarly uninformative (Figure 4-5). However, there was an extremely large difference in the LacZ transducing units between SSTR-2 and SSTR-5 (222 fold, Figure 4-2). If this were due to a higher percentage of virions binding and internalizing in 293/SSTR-5 cells it is likely that that either the standard binding and/or the more sensitive quantitative real-time PCR assay would reflect this. Instead, it is possible that these data reflect non-specific binding [78] and endogenous SSTR-2 receptor expression in 293 cells [79] which, under the typical infection conditions used in this study, do not permit transduction. However, transduction in 293 cells was enhanced under the same conditions as 293/SSTR-2 (see Figure 4-9 legend) indicating that binding and internalization does occur in 293 cells and low levels of infection can be detected under neutralizing conditions. For this reason the amount of

cell-associated virus was normalized to account for virus that may be associated with the SSTR expressing cell lines because of their 293 parentage. When these data are expressed as the fold increase compared to the amount of cell-associated virus in the 293 cells no differences amongst the cell lines were observed that can account for the increased transduction via SSTR-5. 


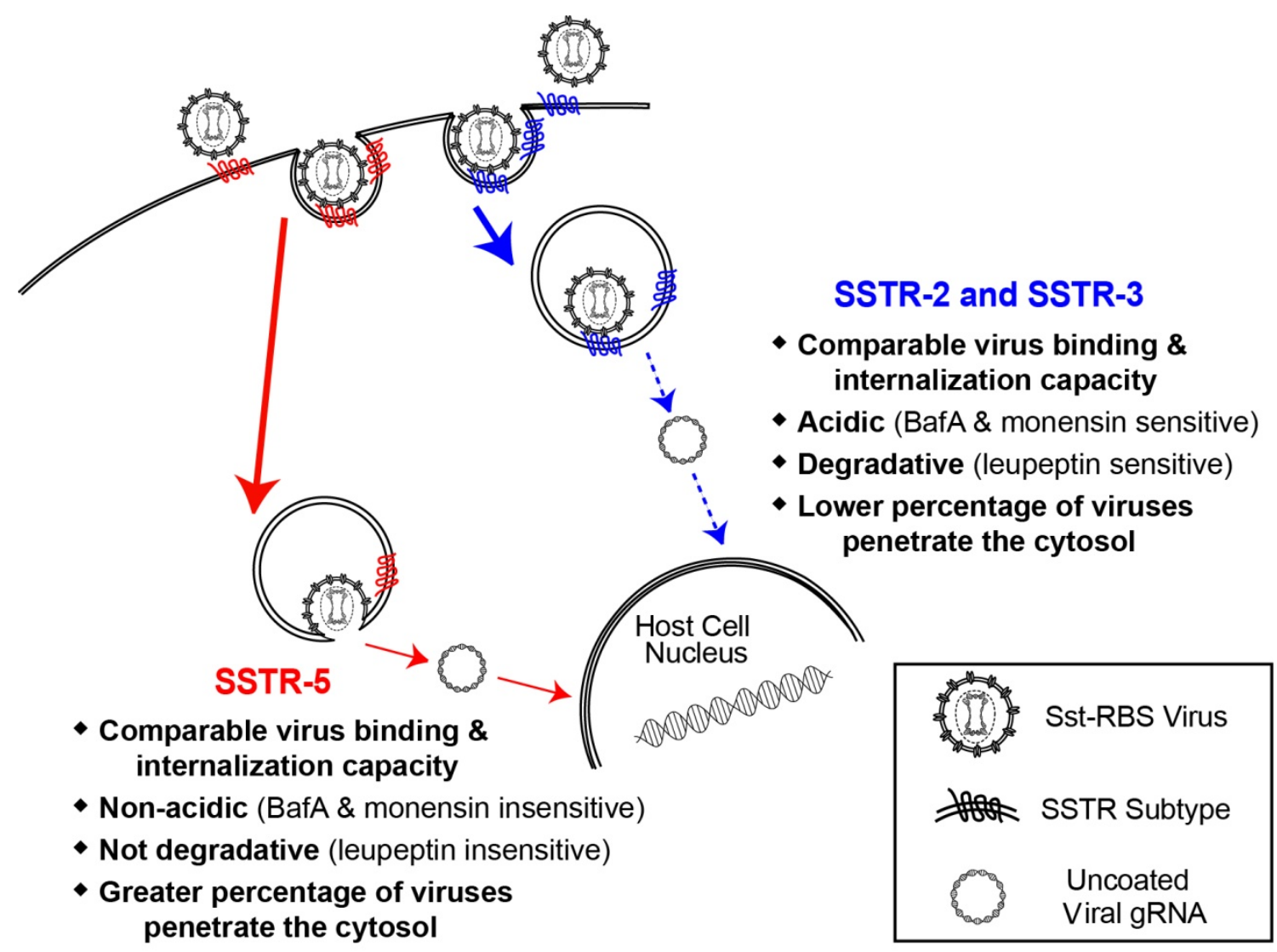

Figure 4-12. Model of the influence of intracellular destination on infection

Thick arrows indicate that comparable amounts of Sst-RBS pseudotyped virus are internalized by each SSTR subtype. Thin arrows indicate that a large percentage of virions complete penetration, reverse-transcription and integration when internalized via SSTR-5. Dashed arrows indicate that a relatively small percentage of virions internalized via SSTR-2 and -3 complete critical late steps of entry into the host cell cytoplasm for infection. The infection characteristics observed for each SSTR subtype are listed next to the corresponding entry pathway. 
If Sst-RBS LV pseudovirions had shown comparable levels of infection among the cell types despite the fact that the MLV pseudotypes were divergent, then this would suggest that the critical step in infection of these virions was dependent on the core particle or genome of the virus (Figure 4-2). On the contrary, these data strongly indicate that the differences in infection are envelope dependent. Since the Sst-RBS glycoprotein is the same in both the LV and MLV pseudovirions and infection was competitively inhibited in each of the SSTR expressing cell lines, the receptor subtype is the most likely variable that could affect such an influence.

Multiple lines of evidence presented here indicate that Sst-RBS pseudovirions internalized via SSTR-5 utilize a distinct intracellular trafficking pattern from that used by SSTR-2 and 3. First, although each of the SSTR-expressing cell lines displayed a dependence on host cell cholesterol for endocytosis, transduction via SSTR-5 was as sensitive to $M \beta C D$ cholesterol depletion as infection of WT MLV via mCAT-1 on NIH 3T3 cells (Figure 4-6C) in which mCAT-1 co-localizes with caveolin during internalization [5]. Second, Sst-RBS pseudovirions internalized at a slower rate in 293/SSTR-5 cells than in the SSTR-2 and -3 lines (Figures 4-7 and 4-8). Third, infection of 293/SSTR-2 and -3 cells increased significantly in the presence of BafA and monensin, whereas infection of 293/SSTR-5 cells was not affected by these inhibitors of vesicular acidification (Figure 4-9A and C). In accordance, HIV-1 infection has been shown to increase in the presence of BafA, an effect that the authors attributed to inhibition of lysosomal acidification that slowed the degradation of virus particles to allow more time for membrane fusion [65]. Viejo-Borbolla and coworkers also proposed a similar explanation for their finding that infection of a modified Moloney MLV glycoprotein retaining the use of mCAT-1 was also increased in the presence of BafA [80].

While I favor that the entry pathway used by Sst-RBS MLV bound to SSTR-2 and -3 exposes virions to a more destructive set of proteases than those entering via SSTR-5, it is also possible that the acidic nature of these compartments may influence the structure of the Env glycoprotein making it more susceptible to inactivation by cellular proteases or less attractive to proteases involved in activating membrane fusion. The extent to which leupeptin inhibited infection of 293/SSTR-2 and -3 cells (Figure 4-10B) indicates the importance of lysosomal proteases in these entry pathways. The parasitic nature of viruses, in particular those such as MLV that rely on host proteases to potentiate the conformational changes required for efficient entry (in contrast to those like VSV whose requirements rely strictly on acidic $\mathrm{pH}$ ), means that they walk a fine line between potentiation and degradation by these same proteases. Raising the $\mathrm{pH}$ of intracellular compartments requires the proteases to function sub-optimally and shifts the dynamic to favor virus entry instead of degradation. However, leupeptin directly inhibits these lysosomal proteases revealing that they are indeed responsible for the results observed in this study. The observation that lysosomal proteases do not appear to be involved in infection via SSTR-5 is further evidence that this receptor delivers virions to a more permissive compartment because it lacks degradative enzymes. The redundant nature of these proteases, limited specificity of available inhibitors and lack of viable knock-out cell lines make it difficult to determine exactly which protease is acting on virus internalized by SSTR-2 and -3. However, possible candidate proteases are most likely in the same class of cysteine proteases as cathepsin B, such as the lysosomal proteases cathepsin $\mathrm{H}$ and cathepsin $\mathrm{L} 1$ since transduction in the presence of the inhibitor CA074 Me showed similar levels of inhibition among the SSTR-expressing cells (Figure 4-10A). Regardless, the results of the drug and protease inhibitor studies, along with the differing rates of internalization during early entry, are consistent with SSTR-2 and -3 
rapidly directing virus to a degradative compartment. In contrast, the patterns observed for SSTR-5 suggest that it internalizes into a non-acidic environment (unaffected by BafA) where it might persist for a longer period of time.

Several alternative interpretations were ruled out for the reasons stated below. First, the increased titer might be explained if SSTR-5 allowed membrane fusion to occur at the cell surface. A precedent for MLV surface fusion was established in rat XC sarcoma cells [81] in which WT-MLV infection was shown to occur independently of microtubule polymerization [43]. However surface fusion is unlikely in 293/SSTR-5 cells since nocodazole inhibited infection indicating that microtubule polymerization is involved (Figure 4-6B), a trait common to most endocytic mechanisms [55]. A second alternative might be that the comparable levels of cell surface receptors detected by flow cytometry using the HA epitope tag did not reflect the actual quantity available to bind virions nor their capacity for internalization. For example, when SSTR-5 was exogenously expressed in COS-7 cells, ligand binding resulted in internalization but surface SSTR-5 was quickly replenished by a combination of mobilization from intracellular pools and receptor recycling [37]. In addition, if the exogenous expression of receptors in 293 cells resulted in a stochastic limitation of the endocytic adaptor proteins, then the capacity of subtypes 2 and 3 for internalization could be compromised. If either condition occurred then a significant difference in the quantity of cell-associated and internalized virions would be expected between the subtypes. However, quantitative real-time PCR of the MSS DNA revealed that similar quantities of Sst-RBS pseudovirions associated with 293/SSTR-2, -3 and -5 cells during a 6 hour exposure (Figure 4-5C), supporting the interpretation that the quantity of effective receptors and their capacity for internalization were comparable amongst the subtypes.

Previous studies suggested that the internalization pathway can influence infection of enveloped and non-enveloped viruses. Under normal conditions, ASLV-A infection is equally efficient using the glycosylphosphatidylinositol (GPI)- and transmembraneanchored isoforms of its cellular receptor TVA [68] and viruses traffic to the same intracellular compartment [69]. However, prolonged exposure of host cells to ammonium chloride alters infection, reducing it for both receptor isoforms but to different degrees, resulting in infection that is relatively greater using GPI-anchored TVA than the transmembrane-anchored isoform [68]. In this perturbed system, viruses were trafficked to different intracellular compartments that were apparently less permissive than the compartment to which the virus normally traffics [69]. However, the ammonium chloridetreated cells showed abnormal intracellular compartment morphology and altered subcellular localization of vesicular trafficking proteins [69] leaving open the question of how much of the observed differences were relevant to natural infection as versus due to pleiotropic effects of the prolonged exposure to ammonium chloride. Other evidence comes from studies of rotaviruses. Strains of these non-enveloped viruses use a number of different cellular receptors, with the choice of receptor being specified by differences in the VP4 spike protein. Interestingly, a study of the strain-specific diversity of VP4 revealed a strong bias toward use of receptors that internalize by clathrin-mediated endocytosis [82]. However, this study did not address if receptors using this endocytic pathway delivered viruses to the same compartment or if the clathrin-dependent bias could have evolved because this pathway is more productive for rotaviruses.

I examined the early entry stages of infection in an effort to more concisely investigate if the intracellular trafficking of internalized virus-receptor complexes affected the efficiency of infection. These studies were begun by generating a set of cell lines that 
provided a system in which the predominant influence on infection would be differences in intracellular trafficking of internalized virus-receptor complexes, thereby circumventing the major technical constraints that have limited previous studies. Regardless of why the intracellular destination varied between receptor subtypes, one subtype delivered virus to a distinct compartment from which a significantly higher percentage of virions penetrated into the host cell cytoplasm. The results directly demonstrate that the intracellular trafficking pathways of a virus receptor can have profound effects on the level of infection. Furthermore, opportunities for more efficient infection presented by intracellular trafficking to more, or less, permissive intracellular environments exerted selective pressure on the choice of a cellular protein as a receptor during viral spike and glycoprotein evolution.

The notion that retroviral glycoproteins and their cellular receptors co-evolved is significant when developing targeted viral vectors. The conformational changes of the Env glycoprotein that are required to initiate infection likely occur only under conditions that mimic the natural virus-receptor relationship. This implies that the placement and structure of the targeting ligand within the glycoprotein are important, but it also suggests that the choice of the targeted receptor should also be carefully considered. This research indicates that targeting to a receptor which internalizes to an intracellular destination with similar properties as the destination of the natural virus-receptor complex will allow for the highest transduction capacity. 


\section{Chapter 5. In Vivo Gene Transduction with Sst-RBS Pseudotyped Lentivirus ${ }^{\S}$}

\section{Introduction}

The capacity to transduce cells endogenously expressing SSTRs in vivo is the next logical step in characterizing the Sst-RBS glycoprotein for use as an in vivo vector. The brain was chosen as the target tissue for these studies because of its immunologically privileged status ensuring that immune responses to the viral vectors would be restricted in the immunocompetent subjects. Additionally, effective treatments have been difficult to attain for the neurodegenerative symptoms of diseases such as the well-known Alzheimer's disease (AD), Huntington's disease (HD) and Parkinson's disease (PD) since drug compounds only mask symptoms and fail to address the underlying causes of these diseases. A related group of diseases which lack treatment for neurodegenerative effects are lysosomal storage diseases (LSD) such as Mucopolysaccharidosis (MPS) VII which results from a deficient $\beta$-glucuronidase (GUSB) enzyme. The deficiency leads to an accumulation of glycosaminoglycans in lysosomal compartments. Affected individuals develop pathologies such as organomegaly, progressive skeletal abnormalities and neurological and cognitive dysfunctions [83] ranging in severity depending on the nature of the enzyme deficiency [84]. Protein and enzyme replacement therapies (ERT), while effective for treating the systemic progression of disease, have had no effect on neurological symptoms due to the extremely limited ability for these treatments to cross the blood brain barrier (BBB) and gain access to the central nervous system (CNS) [8587]. These reasons make neurodegenerative diseases good candidates for treatment with lentiviral directed gene therapies.

SSTR are present in several regions of therapeutic interest in the brain including the choroid plexus (ChP), ependymal cells lining the ventricular system, dentate gyrus of the hippocampus (DG), corpus striatum, substantia nigra and subthalamic nucleus in the brain $[88,89]$ and therefore should be capable of transduction by Sst-RBS pseudotyped lentivirus particles. These regions would also be susceptible to transduction via VSV G pseudotyped vectors but its broad tropism might actually be detrimental in the treatment of these diseases. While it may seem reasonable to use a vector with the ability to transduce nearly all the cells it encounters; this activity works more like a sponge, soaking up large quantities of available vector, preventing its dispersal. In practice, a large area of partially transduced cells may be preferable. Several studies delivering GUSB to brain cells of deficient mice indicate that direct transduction of the GUSB gene to all cells was not required to provide sufficient therapeutic benefit; as disease reversal was observed even in non-transduced cells [90, 91]. The following study is an investigation into the feasibility of in vivo delivery and a comparison of the ability of Sst-RBS and VSV G pseudotyped virions to disperse into the brain parenchyma.

$\S$ Adapted with permission. Li, F., B. Y. Ryu, R. L. Krueger, S. A. Heldt and L. M. Albritton. Targeted entry via somatostatin receptors using a novel modified retrovirus glycoprotein that delivers genes at levels comparable to those of wild-type viral glycoproteins. J Virol, 2012. 86(1): 373-381. 


\section{Results}

\section{Proof of Principle for In Vivo Gene Transduction by Sst-RBS Pseudotyped Lentivirus Particles}

A pilot assay to determine the feasibility of intracranial delivery was performed by microinjecting $2 \mu \mathrm{l}$ of a concentrated stock of HIV-based SIN lentiviral vector bearing either Sst-RBS or VSV G glycoproteins and transducing the red fluorescent protein mKate2 into the subthalamic nucleus of one hemisphere of two mice. Three days post injection the brain tissue was harvested and $20 \mu \mathrm{m}$ frozen coronal sections were analyzed at a magnification of 200X for the presence of mKate2 fluorescence. No red fluorescence was observed in hemispheres that did not receive an injection in either the Sst-RBS or VSV G LV exposed mouse (Figure 5-1A). In the contralateral hemisphere of the VSV G LV injected mouse, numerous red fluorescing mKate2-transduced cells were present along the lower length of the injection pathway or "needle track" (Figure 5-1B). In contrast, the same length of needle track in the Sst-RBS LV injected mouse showed very few red fluorescent cells but a burst of transduction was observed at the end of the needle track in the region of the SN (Figure 5-1C). The area directly above the SN is reportedly negative for SSTR expression [88, 89, 92], however the cellular receptor recognizing VSV G is present throughout these regions [93]. This provides proof principle that Sst-RBS LV does indeed transduce murine brain cells and may represent targeted transduction of SSTR positive cells since a majority of red fluorescence was observed at the end of the needletrack in a region reported to express SSTR [88] while VSV G LV transduced cells in both SSTR expressing and non-expressing regions. The level of transduction observed in the VSV LV injected mice was dramatically higher even in the $\mathrm{SN}$ region. This was to be expected since these lentiviral stocks displayed an approximate 200-fold difference in luciferase TU/ml (Figure 3-1). Based on these observations, the concentrated VSV G LV stock was diluted 100 fold in PBS prior to injection in subsequent experiments.

\section{SSTR-2 Expression in the Murine Brain}

Historically, investigations into the expression of SSTR in the rat and mouse brain have been confined in situ hybridization to localize SSTR subtype mRNA expression or autoradiography using radiolabeled synthetic ligands [88, 92]. More recently, rat models have been used to directly address the level of SSTR surface expression in brain cells with subtype specific antibodies [89]. In an effort to determine where in the mouse brain SSTR surface expression is located, coronal sections were co-stained with a rabbit monoclonal antibody to SSTR-2 and a mouse monoclonal antibody to the neuronal cell nuclear membrane protein, NeuN. Several areas of positive SSTR-2 staining were immediately evident and corresponded to previous reports. These areas were the cerebral cortex layers IV, V and VI; the caudate putamen which comprises the corpus striatum in the mouse; the ependymal cells lining the lateral and third ventricles (Figure 5-2A); the dentate gyrus of the hippocampus and the medial habenula (Figure 5-2B).

Sections were evaluated for co-expression of SSTR-2 and NeuN at a higher magnification (200X). In the cortex (Figure 5-3A) and striatum (Figure 5-3B and 5-3C) the SSTR-2 positive cells co-stain with the NeuN neuronal marker, however not all NeuN positive cells co-stain with SSTR-2 suggesting that, while neurons are the 


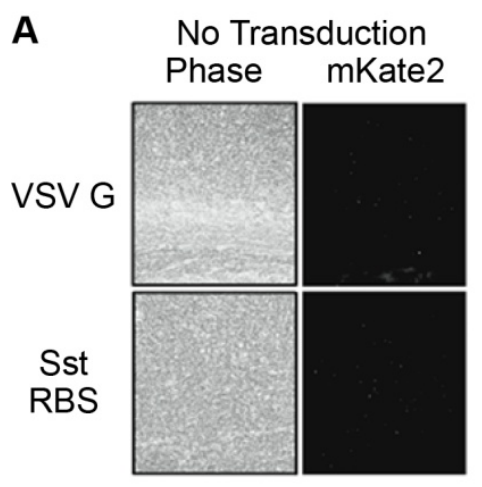

B

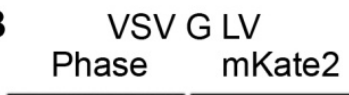

C Sst-RBS LV
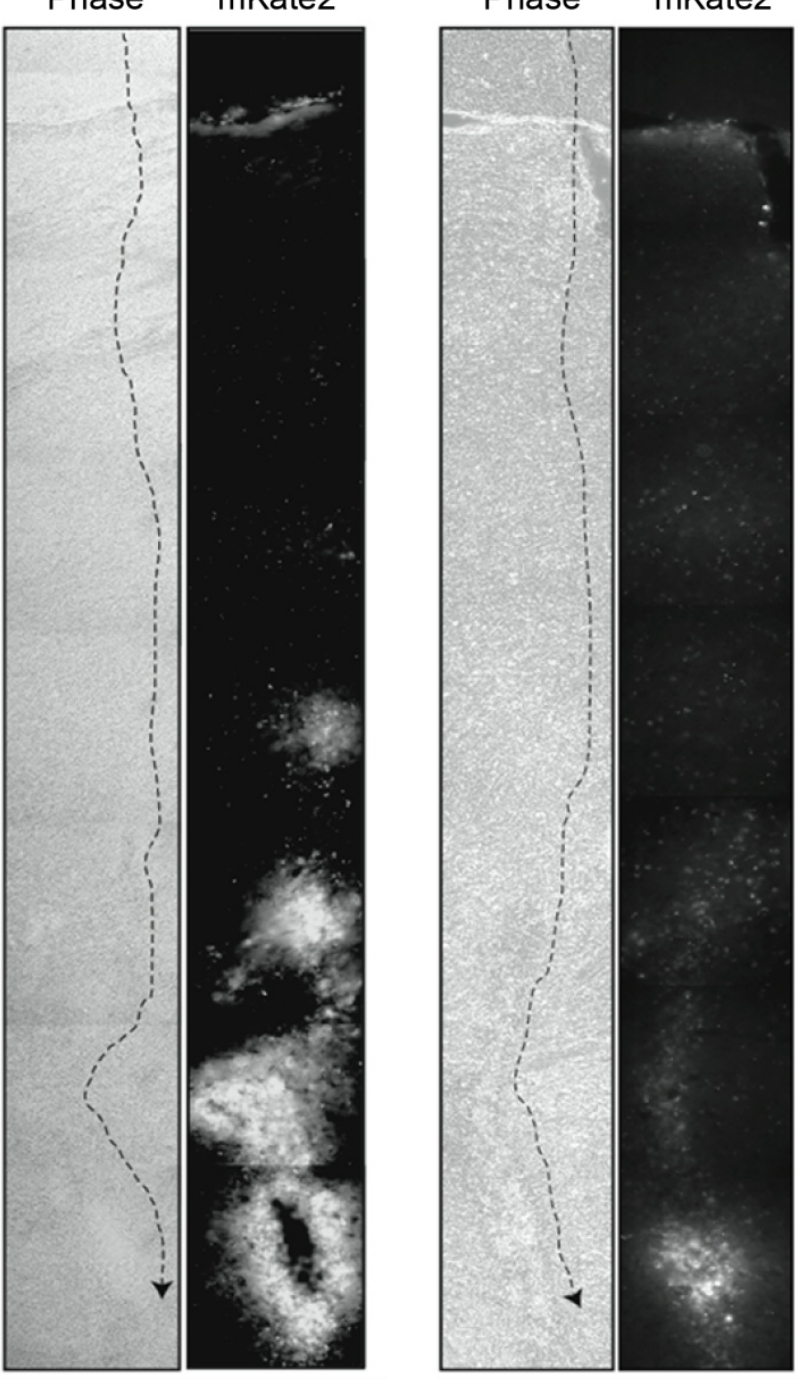

Figure 5-1. Proof of principle for in vivo gene transduction by Sst-RBS pseudotyped lentivirus

Phase contrast images and corresponding mKate2 fluorescence of the uninjected $(A)$ or injected (B and C) hemispheres of mice exposed to VSV G or Sst-RBS lentivirus transducing mKate2. The coronal murine brain section and arrow to the left is presented to indicate the injection trajectory of the representative micrographs at the right. Tissue sections from 9-12 week old mice were microinjected with $2 \mu$ of Sst-RBS or VSV G pseudotyped LV transducing the red fluorescent protein marker, mKate2, in the subthalamic nucleus. Three days later mice were sacrificed and $20 \mu \mathrm{m}$ frozen coronal sections imaged at 200X magnification on a Zeiss Deconvolution microscope. Multiple image frames were carefully aligned to provide a complete image of the needle track. 


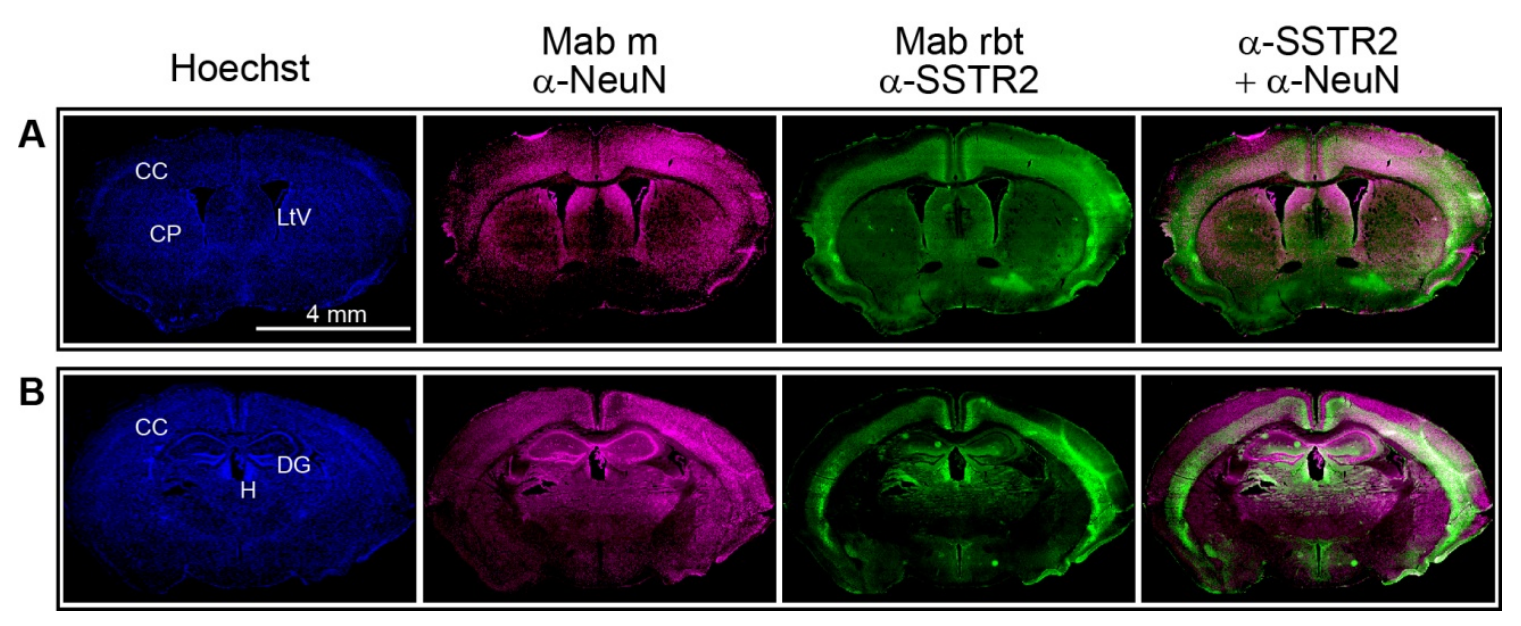

Figure 5-2. SSTR-2 receptors are expressed in the murine brain

$A$ and $B$. Coronal sections through the septo-diencephalic $(A)$ and rostral diencephalic (B) regions from 9-12 week old mice were co-stained with Hoechst nuclear stain (blue), monoclonal mouse anti-NeuN neuronal marker and goat anti-mouse Alexa-647 (purple), monoclonal rabbit anti-SSTR2 and goat anti-rabbit Alexa-488 (green). Micrographs were imaged by Aperio ScanScope at 6X magnification. SSTR-2 positive regions of interest labeled: CC, Cerebral Cortex; CP, Caudate Putamen of the Corpus Striatum; LtV, Lateral Ventricle, DG, Dentate Gyrus of the Hippocampus H, Habenula (medial). 


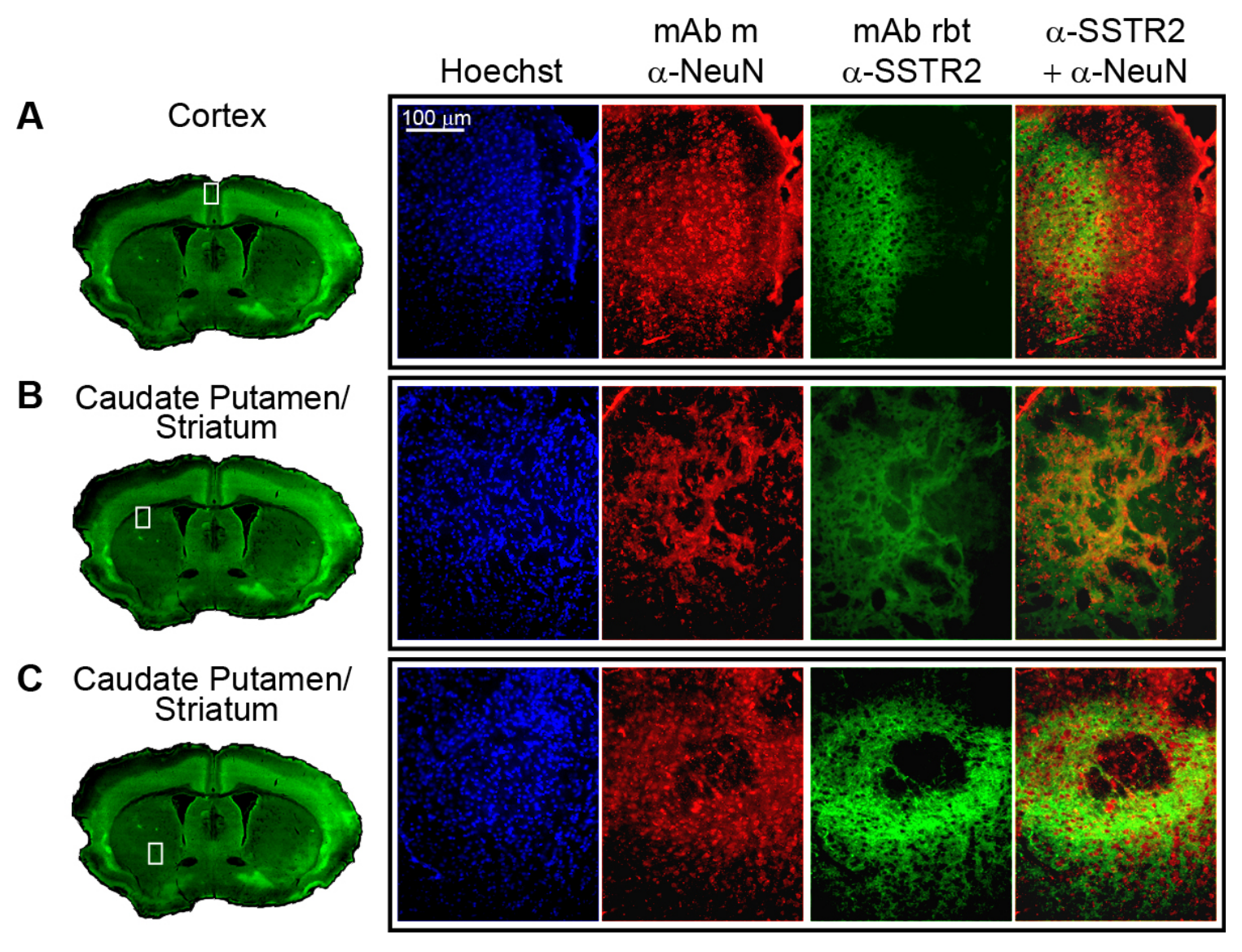

Figure 5-3. SSTR-2 receptors are expressed on neurons of the murine brain

A, B and C. Some, but not all, cortical (A) and striatal (B-C) cells co-stain with the neuronal marker NeuN and surface expression of SSTR-2. Coronal murine brain sections and white boxes to the left are presented to indicate the area from which the micrographs at the right were taken. $20 \mu \mathrm{m}$ frozen coronal sections from 9-12 week old mice were co-stained with Hoechst nuclear stain (blue), monoclonal mouse anti-NeuN and goat anti-mouse Alexa-594 (red), monoclonal rabbit anti-SSTR-2 and goat antirabbit Alexa-488 (green). Micrographs were imaged on a Zeiss Deconvolution microscope at 200X magnification. 
primary cell type expressing SSTR-2 in these areas, only certain neuronal types do so. Interestingly, the ependymal cells lining the lateral (Figure 5-4A) and third ventricles (Figure 5-4B) also express SSTR-2; however, ependymal cells are of a glial lineage. They stain positively for glial fibrillary acidic protein (GFAP) and do not express NeuN [94]. Co-staining sections with antibody recognizing the GFAP and SSTR-2 was not possible during the course of these studies because both primary antibodies were produced in rabbit hosts. Therefore it is possible that other cells of glial lineage may also express surface SSTR-2.

\section{Sst-RBS Lentivirus Mediated Transduction in the Murine Brain}

The lateral ventricle of a mouse injected with $2 \mu \mathrm{l}$ of concentrated Sst-RBS LV vector was evaluated for the presence of mKate2 transduction in cells expressing surface SSTR-2. The injection trajectory of this mouse was originally intended to target the striatum, not the area surrounding the ventricle. However, the variable nature of working with individual subjects is such that this particular injection was sufficiently close to the ventricle that transduction was observed in SSTR-2 expressing cells in the region of the lateral ventricle. This included the NeuN negative ependymal cells (Figure 5-5).

Transduction was also observed in the striatum of mice injected with $2 \mu \mathrm{l}$ of approximately equal luciferase transducing units of Sst-RBS LV or VSV G LV. Representative images of mKate2 transduction along the needle-tracks of both vectors are shown in Figure 5-6A and B. VSV G LV mediated mKate2 transduction was observed to be heavily concentrated around a central core with little diffusion into the surrounding area. In contrast, the core transduction of Sst-RBS LV was less dense but larger in overall area; suggesting that the Sst-RBS LV vector possessed a greater capacity for diffusion in this region of the brain. The area of mKate2 transduction from three Sst-RBS and VSV G LV injected mice was determined using Image J software analysis. The mean area of mKate2 transduction did not differ significantly between the mice injected with Sst-RBS or VSV G pseudotyped vectors, indicating that Sst-RBS pseudotyped lentiviral vectors have a comparable transduction capacity as VSV G vectors in vivo.

The transduction zones (TZ) of multiple sections from three mice injected with $2 \mu \mathrm{l}$ of either the Sst-RBS or VSV G pseudotyped LV vectors were quantified by measuring the height and width of the core and maximum TZ and calculating the ellipsoidal area. The core TZ was defined as the area with the highest concentration of mKate2 expression and the maximum TZ as the largest overall area of mKate2 expression. Additionally, transduction from the contralateral hemisphere of each mouse was measured. These hemispheres were injected with $2 \mu \mathrm{l}$ of either Sst-RBS or VSV G LV vectors immediately after the injection of $0.5 \mu \mathrm{l}$ of $100 \mu \mathrm{M}$ SST-14 peptide, which inhibits Sst-RBS but not VSV G mediated transduction (Figure 4-3). The mean core measurements of both Sst-RBS LV exposed hemispheres were comparable as were the core measurements of the VSV G treated mice in the presence and absence of the peptide. Interestingly, the mean Sst-RBS core TZs were larger than the VSV G cores (Figure 5-7A to E). When the maximum TZs were measured, the untreated Sst-RBS and VSV G zones were comparable in size, but the mean TZ of the hemispheres pre-treated with SST-14 prior to Sst-RBS LV exposure were slightly smaller than their untreated counterparts. As expected, SST-14 pre-treatment had no effect on the maximum TZ area of VSV G (Figure 5-7F to $\mathbf{J}$ ). Even though this was a small sample 


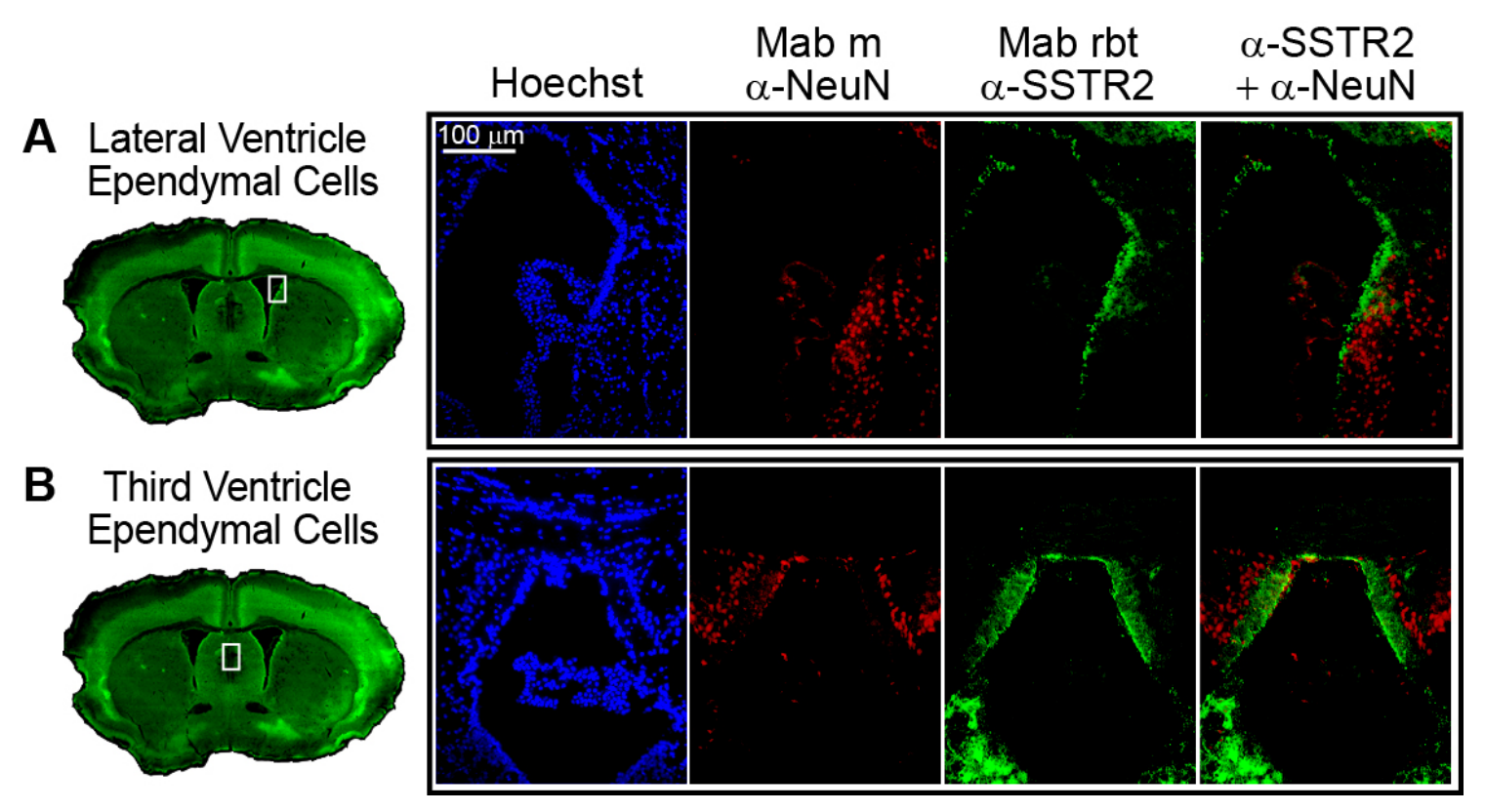

Figure 5-4. SSTR-2 receptors are expressed on murine ependymal cells

$A$ and $B$. Ependymal cells lining the lateral $(A)$ and third ventricles $(B)$ of the murine brain do not stain positively for the neuronal marker NeuN, but express the SSTR-2 receptor. Coronal murine brain sections and white boxes to the left are presented to indicate the area from which the micrographs at the right were taken. $20 \mu \mathrm{m}$ frozen coronal sections from 9-12 week old mice were co-stained with Hoechst nuclear stain (blue), monoclonal mouse anti-NeuN and goat anti-mouse Alexa-594 (red), monoclonal rabbit anti-SSTR-2 and goat anti-rabbit Alexa-488 (green). Micrographs were then imaged on a Zeiss deconvolution microscope at 200X magnification. 


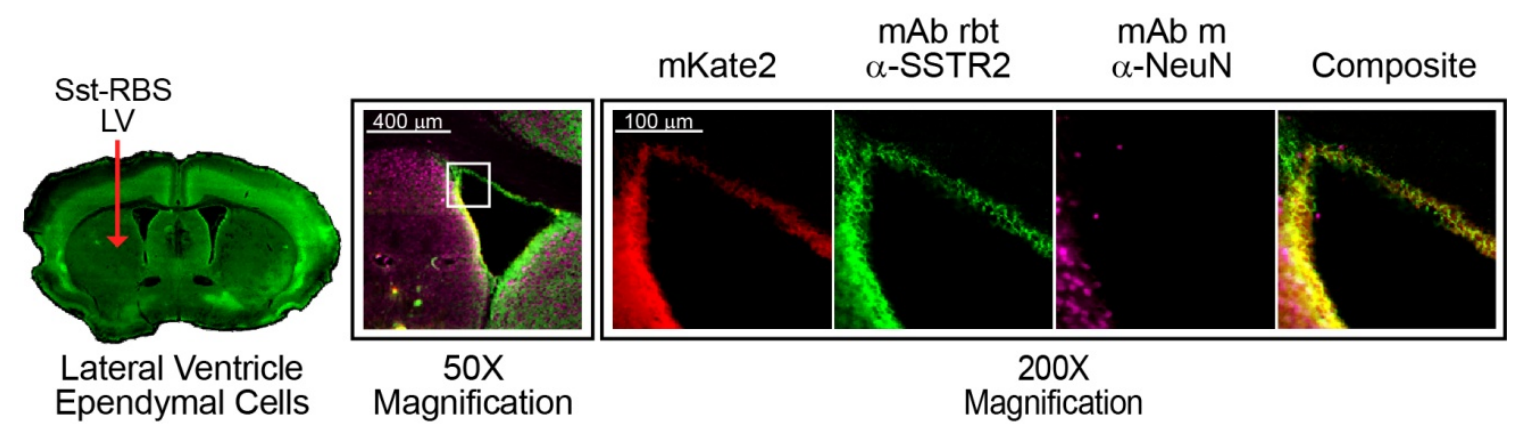

Figure 5-5. Sst-RBS lentivirus mediated transduction of murine ependymal cells

SSTR-2 positive murine ependymal cells colocalize with Sst-RBS-mediated mKate2 marker transduction. The coronal murine brain section and red arrow to the left is presented to indicate the injection trajectory of the representative micrographs at the right. Tissue sections from 9-12 week old mice were microinjected with $2 \mu$ l of Sst-RBS pseudotyped lentivirus transducing the fluorescent protein marker mKate2 (red) in the caudate putamen. Three days later mice were sacrificed and $20 \mu \mathrm{m}$ frozen coronal sections co-stained with monoclonal rabbit anti-SSTR-2 and goat anti-rabbit Alexa-488 (green) and monoclonal mouse anti-NeuN and goat anti-mouse Alexa-647 (purple). Micrographs were imaged by Aperio Scanscope at 50X and 200X magnification. 
A

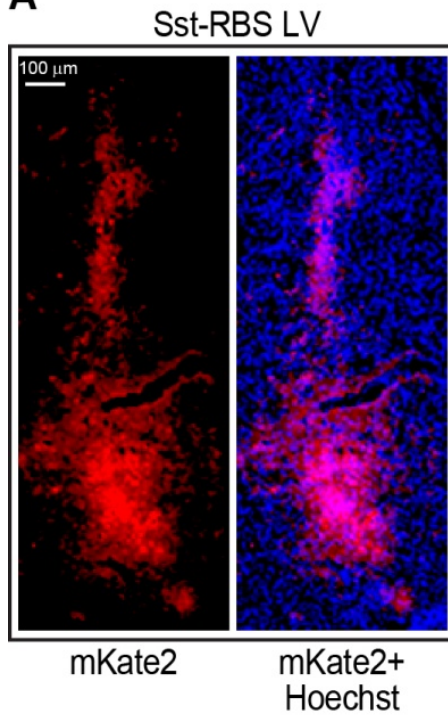

B

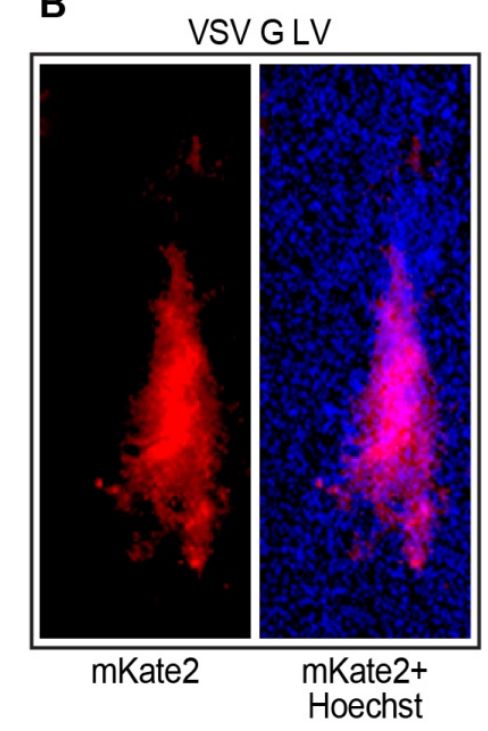

C

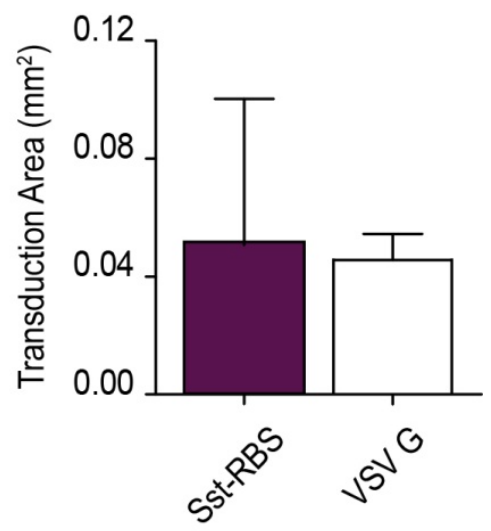

Figure 5-6. Sst-RBS and VSV G lentivirus mediated transduction along the needle-tracks in the striatum of mice

A, B and C. Representative mKate2 (red) transduction zones from Sst-RBS (A) or VSV $\mathrm{G}\left(10^{-2} \mathrm{TU} / \mathrm{ml}\right)(\mathrm{B})$ pseudotyped lentivirus microinjected into the caudate putamen of 912 week old C57bl/6 mice. Three days later mice were sacrificed and $20 \mu \mathrm{m}$ frozen coronal sections were stained with Hoechst nuclear stain (blue) and micrographs imaged by Aperio Scanscope at 34X magnification. (C) The area of mKate2 transduction from three Sst-RBS LV (purple) or VSV G LV (white) injected mice was determined using Image $\mathrm{J}$ software. The mean and SD of mKate2 transduction is shown $(n=3)$. 
Figure 5-7. Transduction zone diffusion of Sst-RBS and VSV G lentivirus particles in intracranially injected mice

A through L. Mice were microinjected in the caudate putamen with $2 \mu$ of concentrated mKate2 transducing lentivirus coated with Sst-RBS or VSV G $\left(10^{-2} \mathrm{TU} / \mathrm{ml}\right)$ in the presence or absence of $100 \mu \mathrm{M}$ SST-14 ligand. Three days post infection, the mice were sacrificed, and $20 \mu \mathrm{m}$ frozen tissue sections were analyzed for mKate2 expression (red). Aperio Scanscope images were measured for core (A-E) and maximum (F-J) transduction zone areas. The mean $\pm S D$ for three mice are shown in $A$ and $F$. Representative images for Sst-RBS (B,C, G, H) and VSV G (D,E,I,J) zones are shown indicating mKate2 expression as well as areas of no transduction from comparable sections for Sst-RBS (K) and VSV G (L).

One Way ANOVA and Bonferroni posttest used to determine P-value > 0.05; NS, Not Significant. 


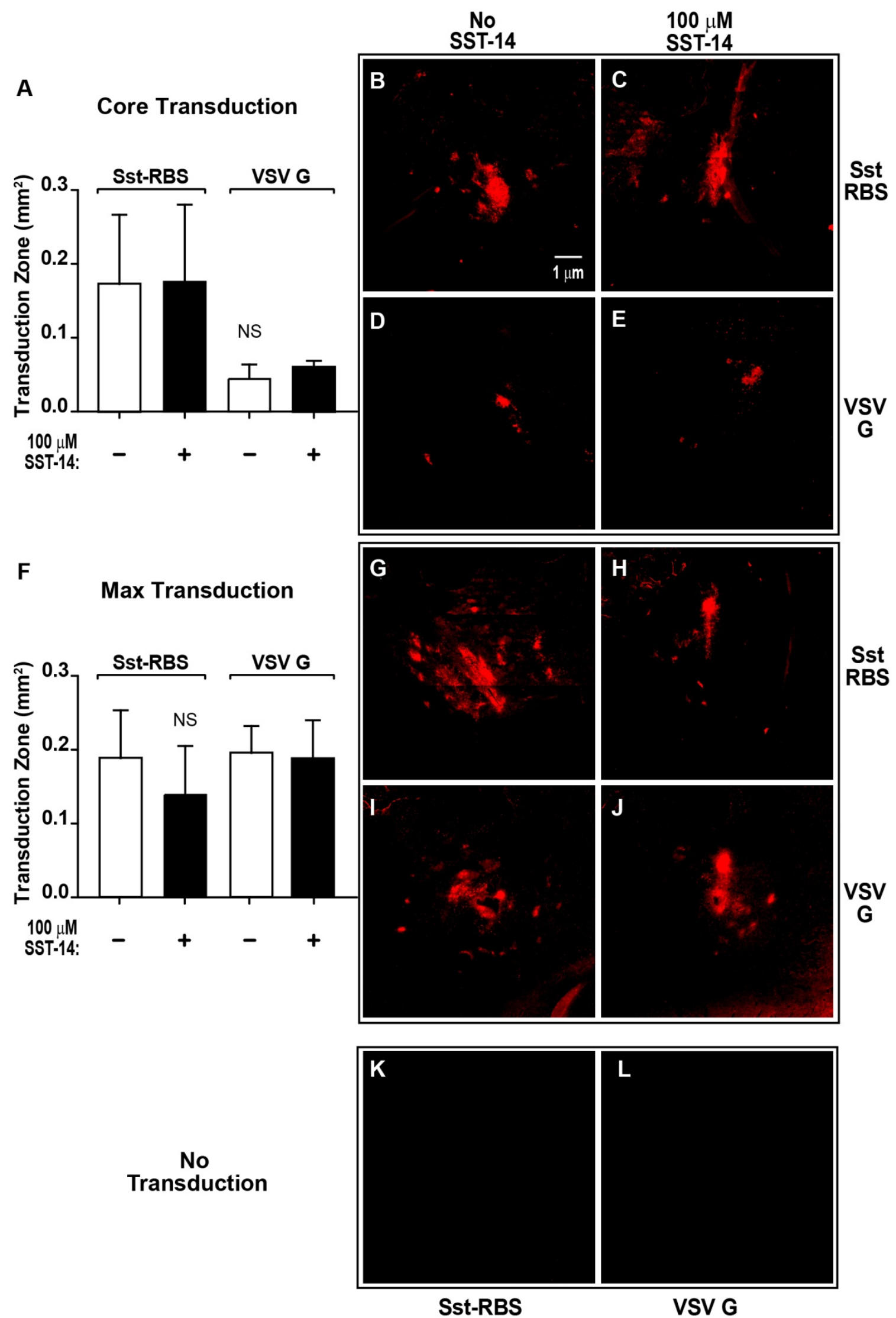


size $(n=3)$, some guarded conclusions could be drawn from these observations. First, the larger core TZ area of Sst-RBS LV suggests that it was capable of diffusing away from the injection site into the surrounding brain tissue while the VSV G LV particles were immediately adsorbed onto cells in the vicinity of the injection site. Second, the comparable maximum TZs of hemispheres exposed to virus in the absence of SST-14 suggest that VSV G LV particles escaping adsorption at the injection site were capable of diffusing the same distance as Sst-RBS LV particles. In general, however, the VSV G TZs had a more distinct core and less distinct maximum area; whereas, the Sst-RBS TZs were more uniform in intensity throughout both measurement areas. Third, the decrease in maximum TZ area of Sst-RBS LV in the presence of SST-14, although not statistically significant, can be interpreted to indicate that transduction was specifically mediated by the presence of SSTR.

\section{Discussion}

This study demonstrated proof of principle for in vivo Sst-RBS mediated transduction in the murine brain (Figure 5-1) which displayed comparable transduction efficiency to VSV G pseudotyped vectors (Figures 5-6 and 5-7). Several regions of interest in the murine brain stain positively for SSTR-2 surface expression (Figure 5-2) and SSTR-2 is expressed on some classes of neurons (Figure 5-3) as well as ependymal cells which line the ventricular system (Figures 5-4 and 5-5). These observations suggest several areas of potential clinical relevance for the chimeric SstRBS glycoprotein. The subthalamic nucleus is involved in PD and has also been implicated in AD [95]. Additionally, transduction of cells in the striatum indicates that this new chimeric glycoprotein may be useful as a therapeutic and investigational tool for $A D$ and $\mathrm{HD}$ as well as other neurodegenerative diseases involving these regions of the brain such as LSDs.

Enzyme replacement therapies (ERT) have been developed for several LSDs including Gaucher disease, Fabry disease, Pompe disease, MPS I, MPS II and MPS VI $[86,96]$ based on the discovery that a small amount of proenzyme is naturally excreted during transport from the TGN to lysosomes and can be taken up either by the original or a neighboring cell by the mannonse-6-phosphate receptor (M6PR) [97, 98]. However, with the exception of Gaucher disease [86, 99], ERT has only been moderately successful since it requires life-long treatments of exogenously produced recombinant enzymes [87] and because intravenously administered enzyme is inefficiently delivered to target cells. A majority of recombinant enzymes collect in reticuloendothelial cells of the liver and spleen [86] and are incapable of crossing the BBB for delivery to the CNS $[86,96]$. The latter is a particular concern for LSDs that manifest with severe neurodegeneration such as MPS VII. Directly injecting enzyme into the cerebrospinal fluid (CSF) may be a means of circumventing the BBB, but this approach still remains impractical due to the long-term nature of ERT [86].

Advances in gene therapy are a promising direction for the treatment of neurological diseases and LSDs. Both systemic and direct CNS delivery of viral vectors including adenovirus (ADV), adeno-associated (AAV), RV and LV vectors have successfully transduced target genes providing long-term protein expression (for review see [96]). RVs and LVs are particularly useful due to their ability to integrate into host chromosomes thus alleviating the continious nature of ERT. Typically, intravenous delivery of GUSB expressing vectors results in transduction of liver and spleen cells 
which are then capable of secreting enough cross-protective enzyme into the bloodstream that therapueutic benefits were observed in the peripheral organs and skeleton [100-103]; however, transport across the BBB is not evident [96, 100, 102]. In contrast, direct injection into the CNS has been shown to be effective at long term expression of marker and therapeutic genes by ADV, AAV and VSV G pseudotyped RV and LV delivery methods [90, 91, 96, 104]. While ADV, AAV and LV particles were capable of transducing both NeuN postive neurons and GFAP postive glial cells (VSV G pseudotyped RV was not capable of transducing the terminally differentiated neurons), VSV G pseudotyped LV was by far the most efficient. The LV transduced more cells $/ \mathrm{mm}^{3}$ than the other vectors with approximately $89 \%$ of all transduced cells identified as NeuN positve neurons [105]. The present study suggests that the Sst-RBS targeting LV is at least as capable of trandsduction as the VSV G LV when approximately equal TU/ml were compared (Figure 5-7F). Moreover, the Sst-RBS LV may be preferrable. Due to its targeted nature it displayed a greater potential for diffusion away from the initial injection site (Figure 5-7A).

SSTR mRNA and surface expression have been demonstrated throughout the murine and rat brain. Whereas SSTR-2 and -3 are the predominant subtypes expressed, SSTR-1 and -4 are also expressed and to a far lesser extent SSTR-5. [88, 89, 92]. This study only investigated the expression of SSTR-2 in the murine brain as an initial indication of feasibility. Since the SST-14 ligand displayed by the Sst-RBS glycoprotein is equally capable of binding and internalizing through subtypes 2, 3 and 5 (Figure 4-5), transduction might occur via any of the SSTR subtypes in vivo.

It was surprising to note that Sst-RBS mediated transduction was as efficient in vivo as VSV G (Figure 5-6) given that SSTR-5 expression in the brain is reportedly extremely low and in vitro transduction was significantly greater via this subtype than SSTR-2 and3 (Figure 4-2), the predominant subtypes expressed in the brain [88, 89]. This suggests that, at least in the brain, in vitro studies may not correlate with in vivo transduction. Since the presence of only one SSTR subtype, SSTR-2, was evaluated during this preliminary investigation; this level of Sst-RBS mediated transduction might represent the use of several SSTR subtypes as multiple SSTR expression has been reported [88, 89]. Further studies will need to be performed in order to assess the expression of each SSTR subtype in lentivirus transduced brain cells. Additionally, the highly specialized nature of neurons may further account for this disconnect. Their unique shape and size means that their intracellular trafficking patterns are prolonged compared to other cell types [106, 107]. Moreover, their low turn-over rate, limited access to extracellular nutrients and need to heavily regulate their membrane and cytoplasmic contents set extreme limits on cellular resources. One of the many ways neurons have developed to compensate for these requirements is a highly ordered endosomal and lysosomal system. [106-108]. Indeed, the lysosomal compartments of neurons are exquisitely sensitive to perturbation as evidenced by their involvement in almost every neurodegenerative disease including those discussed above. In an effort to conserve resources, neuronal cells often slowly recycle signaling receptors such as SSTRs instead of downregulating them through lysosomal degradation [107, 108]. In agreement with this, Csaba and colleagues demonstrated retrograde transport and recycling of SSTR-2 in hippocampal neurons. Agonist induced SSTR-2 internalization was achieved through rapid endocytosis to early-endosomes, bypassing the low $\mathrm{pH}$ compartments it accumulated in a perinuclear region consistent with the TGN before slowly recycling back to the plasma membrane [109]. Evidence that trafficking to acidic environments inhibited transduction in 293/SSTR-2 and -3 cells (Figure 4-9) supports that the 
neuronal SSTR-2 trafficking pathway might provide a higher level of transduction than in in vitro systems. Additionally, the elongated intracellular route that the virus-SSTR complex travels may provide the time necessary for membrane fusion and penetration events to occur. 


\section{Chapter 6. Discussion}

\section{Identify the Endocytic Mechanisms and Intracellular Destination of Sst-RBS Entry}

In this body of work I demonstrated that MLV and HIV-1 based virion particles pseudotyped with the Sst-RBS glycoprotein were trafficked to at least two different intracellular compartments in 293 cells expressing SSTR subtypes 2, 3 and 5. Moreover, the intracellular environment to which SSTR-5 was directed was more permissive than the environment that SSTR-2 and -3 directed viruses. This investigation into receptor characteristics is significant in that it directly demonstrates that differences in virusreceptor trafficking can profoundly influence infection efficiency. Endocytosis of the somatostatin receptor subtypes 2, 3 and 5 has previously been shown to be clathrindependent $[28,37]$. Yet several lines of evidence suggest that virus bound to SSTR-5 was internalized by a distinct mechanism than SSTR-2 and -3; moreover, this functional difference in endocytosis directly correlated with a higher level of infection. Further experiments to address which endocytic mechanism Sst-RBS uses for internalization via these receptors, identify which intracellular locations these virus-receptor complexes are directed and how they influence virus entry would be beneficial for a complete characterization of Sst-RBS mediated entry; as well as determining how useful particular endocytic and intracellular pathways may be to targeting gene delivery.

To further investigate the endocytic mechanism of Sst-RBS mediated entry, dominant negative (DN) Rab GTPases can be utilized. Rab proteins are members of the Ras GTPase superfamily and are the master regulatory elements of endosomal transport and vesicular trafficking within cells. They act as guanine nucleotide-dependent switches which further recruit cascades of effector molecules [110,111]. Together these mechanisms sort cargo, control the directionality of vesicle movement and govern membrane fusion between vesicles [112]. Over 60 Rabs have been identified in humans thus far [112]. Rab5, Rab7 and Rab11 especially, have been extensively studied for their pivotal roles in endocytosis and have become the defining markers for the early (EE), middle/late (LE) and recycling (RE) endosomes, respectively. Cells transiently expressing a DN-Rab5 protein have been shown to be defective for fusion of primary endocytic vesicles from the plasma membrane with the EEs, and DN-Rab7 prevents the fusion of EEs and LEs blocking the pathway to lysosomes. Recently Rab11 depletion was shown to inhibit the fusion of REs with the plasma surface [113]. Whereas Influenza A virus infection was inhibited by the expression of both DN-Rab5 and DN-Rab7 (indicating the involvement of the LE) infection of VSV and Semliki Forest virus were only inhibited by DN-Rab5 expression indicating the involvement of only the EE compartment for these two viruses [114, 115]. Additionally, DN-Rab11 had no effect on infection of either Influenza or VSV viruses [114]. From these results I would predict that Sst-RBS infection via each SSTR should be inhibited by DN-Rab5 expression; however, would expression of DN-Rab7 increase infection via SSTR-2 and -3 by blocking the degradative pathway? Also, if the RE is involved in increasing the effective number of SSTR-5 receptors, an additional question that might be answered is would inhibiting the recycling pathway with DN-Rab11 also inhibit infection via SSTR-5? To test these questions, the SSTR-expressing cell lines would be transiently transfected with one of the DN-Rab expression plasmids in which the Rab protein is fused with a GFP marker. These cell populations (as well as populations transiently transfected with WT Rab constructs fused to GFP) would then be exposed to Sst-RBS, WT and VSV G pseudotyped MLV particles. At $48 \mathrm{hpi}$, when $\beta$-galactosidase expression is evident, the 
cells will be fixed and permeabilized. An anti- $\beta$-galactosidase antibody followed by a secondary antibody conjugated to Alexa-594 will be used to probe for transfected cells so that both GFP and $\beta$-galactosidase expression can be visualized simultaneously. At least 100 hundred individual cells would then be evaluated for co-expression of GFP and $\beta$-galactosidase. If transduction is inhibited by the loss of Rab activity, a decrease in the number of cells that are both GFP and $\beta$-galactosidase positive will be observed compared to the WT Rab transfected SSTR-expressing cells.

Dependence on the presence of Rab5 and Rab7 could also be used to investigate the endocytic mechanism by which Sst-RBS-SSTR subtypes are internalized. Clathrin-mediated endocytosis shows dependence on both Rab5 and Rab7 while Caveolin-associated endocytosis only shows dependence on Rab5 [55]. Several pathways in addition to clathrin- and caveolin-dependent endocytosis are utilized by viruses for the purpose of gaining entry to the intracellular milieu. Of particular interest is the macropinocytic pathway which typically involves microtubules, cholesterol, Rab5, and Rab7 as well as other cellular processes like $\mathrm{Na}+/ \mathrm{H}+$ exchange, which decreases the $\mathrm{pH}$ of pre-lysosomal and lysosomal vesicles. A growing number of viruses have been shown to be internalized in macropinosomes including vaccinia virus, adenovirus serotype 3 , echovirus 1 , coxsackivirus $B$, herpes simples virus 1 , and has been implicated in HIV-1 infection [116]. Recently, ebola virus was also added to this list [117, 118]. If infection of Sst-RBS shows dependence on both Rab5 and Rab7 the use of a phosphatidylinositol-3-kinase (PI3K) inhibitor such as wortmannin to inhibit ruffle formation might help distinguish clathrin-mediated from macropinosome internalization.

I demonstrated that there was a significant block to entry of Sst-RBS pseudotyped virions via SSTR-2 and -3 which occurred after internalization but prior to reverse transcription (Figure 4-11). A block of this type is often seen when virions fail to trigger membrane fusion [12, 14, 46]. Membrane fusion of the WT MLV Env is accomplished by first binding to its cellular receptor that, in turn, causes conformational changes between the TM and SU subunits. Upon internalization, host cell proteases further potentiate membrane fusion by cleaving the MLV Env, allowing conversion from a hemi-fusion to a fusion state, which subsequently allows pore formation and virion core penetration $[7,8]$. The Sst-RBS virus is capable of efficient membrane fusion when bound to and internalized by SSTR-5, but are the possible fusion defects seen in 293/SSTR-2 and -3 cells because the correct TM-SU conformation changes were not achieved or because the compartment to which these receptor subtypes direct the virions is non-permissive? Evidence that virions bound to SSTR-2 and -3 were capable of inducing membrane fusion was seen when infection was carried out in the presence of BafA and monensin which neutralize endosomal compartments (Figure 4-9); indicating that the block is due to the nature of the compartments. Direct demonstration that SSTR-2 and SSTR-3 are fusion-competent can be achieved through a cell-cell fusion assay in which mixing of the outer leaflet of the cell membrane is compared to cytoplasmic content mixing. Effector 293 cells exogenously expressing the Sst-RBS glycoprotein and a cytoplasmic dye are mixed with target 293/SSTR-expressing cells containing a differently colored cytoplasmic dye and DiI, a lipid soluble dye as a membrane marker. In previous infection experiments, it was noted numerous times that 293/SSTR-expressing cells do not form syncytia in the presence of Sst-RBS virus; however, the cells do show a higher order of cell-cell attachment that is typically seen immediately prior to syncytia formation (anecdotal evidence). These cell-cell attachments might indicate that the virus is trapped in hemi-fusion state and incapable of completing membrane fusion because they have 
not been exposed to host proteases. If this is the case, one would expect some degree of lipid mixing between the outer plasma membrane of DiI positive target cells and negative effector cells. To induce membrane fusion and content mixing, a protease may need to be added to the cells as a final trigger. Possible outcomes of this experiment might indicate that each SSTR is capable of mediating the same amount of membrane fusion after the protease trigger; or alternatively, that Sst-RBS-SSTR-5 complexes are more fusigenic. Additionally, while the complexes may be triggered by the same protease they might be sufficiently distinct that the protease that triggers the SSTR-5 complex is different from the protease required by SSTR-2 and -3 . If the latter is true then identifying which proteases are capable of triggering membrane fusion may also provide clues to the intracellular location that each of these receptors directs internalized viruses. Knowledge gained from this information would build a better foundation for creating viral vectors that match the entry requirements of the targeted entry receptor.

\section{Sst-RBS Lentivirus Titers}

A particular unexplained observation was that while the Sst-RBS LV titers showed the same general trend of transduction in vitro, namely that transduction of 293/SSTR-5 cells was higher than 293/SSTR-2 and -3 cells, the overall transduction of Sst-RBS LV compared to the equivalently pseudotyped MLV was much lower (Figure 4-2). Yet this does not seem to translate into low titers during in vivo experiments (Figure 5-6) in the mouse brain. This might be due to a relatively low number of glycoprotein spikes incorporated by the HIV-1 virion in its membrane. Typically, HIV-1 incorporates between 8-10 trimers per virion [119]. Others demonstrated that whereas low levels of Env incorporation was sufficiently capable of mediating transfection, artificially enhancing the amount of Env increased transduction but only to a certain extent [120]. Additionally, retrovirus Env pseudotyped LV particles showed limited levels of incorporation [48, 49] and transduction [23] which may also be a factor in decreasing the overall LV titers.

A further explanation may be that a host restriction factor is acting on either or both the MLV and LV particles. Multiple host restriction factors have been identified and characterized in humans, mice and non-human primates with varying restrictive activities for retroviruses as well as a number of other viruses. Of the currently identified host restriction factors, the most pertinent to the systems described in this research are the APOBEC3, TRIM5 $\alpha$, Fv1 and the recently identified Tetherin and SAMHD1 proteins [121]. Restriction of viruses by these host factors occurs by one or a combination of the following: preventing virion budding, uncoating, reverse transcription of viral RNA, or nuclear import/integration mechanisms.

APOBEC3 (Apolipoprotein B mRNA-editing enzyme catalytic polypeptide-like 3) proteins belong to the cytidine deaminase family. Humans encode 6 proteins $(A, B, C$, $\mathrm{DE}, \mathrm{F}, \mathrm{G}$ and $\mathrm{H}$ ) whereas mice encode a single protein. The mouse APOBEC3 protein and the human $G$ and $F$ proteins can have restrictive activity on HIV-1 infection. They are packaged into virus particles by producer cells and are transferred to target cells during infection and inhibit viral replication by cytidine deaminase-dependent or -independent mechanisms. In the dependent mechanism, viral cytosines are deaminated to generate uracils on newly synthesized minus-strand cDNA. The presence of uracils in DNA either triggers DNA repair enzymes to degrade the product or they generate $G$ to $A$ hyper-mutations in the copied plus-strand DNA that negatively affect the integrity of the viral genomic content $[121,122]$. APOBEC3G and $F$ can also reduce the 
efficiency of tRNA ${ }^{\text {lys3 }}$ priming of the viral RNA and block reverse transcription as well as block viral integration in deaminase-independent mechanisms that are not well understood [121]. HIV-1 has developed an accessory protein; Vif (viral infectivity factor) that is capable of binding APOBEC3 proteins and linking them to E3 ligases which induces polyubiquitylation of both APOBEC3 and Vif triggering proteasomal degradation $[113,114]$. Vif is also capable of blocking APOBEC3 protein from encapsulation by an unknown degradation-independent mechanism [121]. Thusly, APOBEC3G and F should not be capable of restricting the Vif-positive HIV-1 infection in the human 293-derived SSTR-expressing cell lines. Interestingly, Vif activity is species-specific; but HIV-1 Vif should also be capable of blocking and counteracting APOBEC3G and F proteins during the in vivo mouse experiments because their activity is entirely dependent on packaging in virions prior to release. Since the producer cell line (293FT) used to generate the Sst-RBS and VSV G pseudotyped LV particles for these experiments is a human line, the species-specificity is still guaranteed. Additionally, human APOBEC3 proteins do not restrict MLV viruses [122].

Tetherin is constitutively expressed on the surface of bone-marrow CD34+ cells as well as B and T cells. It is an unusual membrane protein in that it is doubly anchored at the cell surface by a glycophosphatidylinositol (GPI) anchor in cholesterol-rich lipid microdomains and an $\alpha$-helical transmembrane domain located outside lipid rafts. Tetherin's restrictive activity most likely derives from a homodimeric structure which anchors budding virions via the GPI-anchor at virus assembly sites in lipid microdomains and "tethers" them to the surface via the transmembrane domain inhibiting the release of the budding virions. The HIV-1 accessory protein $\mathrm{U}(\mathrm{Vpu})$ is capable of binding to and down-regulating tetherin from cell surfaces possibly by proteasomal degradation [121]. The recently described SAMHD1 (sterile alpha motif and histidine-aspartic domain) protein is expressed in myeloid cells, T and B cells. It restricts HIV-1 and MLV replication by decreasing the intracellular pool of dNTPs in non-dividing cells [121]. However, no expression of either of these two host factors has been documented in human 293 cells or murine brain cells.

The human tripartite motif (TRIM)-containing protein $5 \alpha$ isoform shows crossspecies-specific restriction of retroviruses, as this restriction factor does not restrict viruses isolated from the same host species (e.g. human TRIM5 $\alpha$ does not restrict HIV-1). TRIMs have multiple functional domains; an N-terminal RING finger domain, one or two B-box domains, a coiled-coil domain and many also have a C-terminal PRYSPRY domain. This PRYSPRY domain is essential for interacting with retroviral CA proteins; but only when the capsid is in a lattice structure [123]. The RING domain of TRIM5 $\alpha$ functions as an E3 ubiquitin ligase and the B-box and coiled-coil domains function together in protein-protein interactions allowing TRIM5 $\alpha$ to multimerize around the retroviral CA lattice. The full nature of TRIM5 $\alpha$ restriction is still not entirely identified, but in its presence restricted retroviral capsid structures are prematurely disassembled and the viral reverse transcription complex is proteasomally degraded [121]. Indeed, recently TRIM5 $\alpha$ was found to associate with PSMC2, a proteasomal subunit, in discrete cytoplasmic bodies [124]. While this association occurs in the absence of restricted virus, in its presence proteasomal association with TRIM5 $\alpha$ cytoplasmic bodies increased [125]. There is also evidence to suggest a non-proteasomal action of TRIM5 $\alpha$ in which TRIM5 $\alpha$ blocks restricted CAs from interacting with host machinery and thereby blocking nuclear import [126]. As the PRYSPRY domain of human TRIM5 $\alpha$ does not recognize HIV-1 or Moloney MLV CA proteins, it is unlikely that TRIM5 $\alpha$ is restricting 
transduction in the SSTR-expressing 293 cells. Although mice do not express TRIM5 $\alpha$, they do have a similar host factor termed Friend virus susceptibility factor-1 (Fv1). One of three $\mathrm{Fv} 1$ alleles $\left(\mathrm{Fv} 1^{\mathrm{n}}, \mathrm{FV} 1^{\mathrm{b}}\right.$ or $\left.\mathrm{FV} 1^{\mathrm{nr}}\right)$ are expressed by in-bred mice and they each encode a degenerate retroviral Gag protein which binds the N-terminal domain of CA from different MLV strains. This interaction restricts MLV infection at a point after reverse transcription but before integration; however the action by which this occurs has not been elucidated [127]. Neither Moloney MLV nor HIV-1 is restricted by any of the Fv1 alleles [122].

Neither the available literature nor the experimental results indicate that a host restriction factor might be acting to reduce transduction by SSTR-2 or -3; nevertheless, the possibility is not excluded. The issue is of particular relevance if one considers that virions associated with SSTR-2 and -3 may not be inactivated in lysosomes as proposed, but instead these receptors are sorted to a compartment where membrane fusion may be just as efficient as with SSTR-5 and yet uncoating/penetration is still somehow restricted. However, this means that the host factor must be specifically located and accessible only by certain entry routes, blocking virions entering through SSTR-2 and SSTR-3 but not through SSTR-5. If such a host restriction factor is involved its identification will require further research efforts such as siRNA mediated knockdown of the restriction factors discussed here. Transduction assays could then be performed in the presence and absence of each respective host factor and differences in transduction evaluated.

\section{Targets for Sst-RBS-Mediated Gene Delivery In Vivo}

\section{SSTR-expressing Tumors}

SSTRs have a broad distribution throughout the body. Surface expression of SSTRs is abundant in the brain; gastrointestinal tract; exo- and endocrine pancreas and pituitary $[27,28]$. SSTRs have also been identified on the surface of neuroendocrine tumors (NET) [128], breast cancer tumors [129] glial tumors and meningiomas, renal cell carcinomas and lymphomas [130]. NETs occur most frequently in the gastrointestinal tract (in descending order of frequency: small intestine, rectum, colon, pancreas, stomach and appendix) but also in the bronchopulmonary system, ovaries, testes and hepato-biliary system with much lower incidences. A majority (70-90\%) of NETs express multiple SSTRs with SSTR-2 and SSTR-5 subtypes being the most common [128]. Similarly, SSTR-2 expression is predominant in breast tumors, however, multiple SSTR subtypes are also displayed [129]. Radio- and generator-derived positron emitter-labeled somatostatin analogs (SSA) allow visualization of tumor lesions with nuclear imaging techniques for diagnosis, tumor localization and grading [128]. SSAs themselves have also been used for a variety of treatment outcomes. They have been shown to control tumor growth, inhibit the secretion of hormones and growth factors, control hormone-related symptoms and in some cases cause tumor shrinkage [131]. Additionally, cytotoxic drugs (such as camptothecin, methotrexate, paclitaxel, and doxorubicin) conjugated to SSTR-2 specific analogs show positive treatment outcomes [132] and Octreotide induced SSTR-2 internalization in NETs has been directly demonstrated [133]. However, decreased expression of SSTRs in dedifferentiated tumors associated with metastatic states of both NETs and breast cancers have been documented [128, 129]. Additionally, target receptor signaling and internalization 
pathways may become interrupted in tumor cells [134] suggesting that SSA-related diagnosis and treatment has a limited window of efficacy and early diagnosis of patients is critical. The addition of Sst-RBS vector treatments transducing any number of chemotherapeutic or cytotoxic genes as well as genes rendering tumors susceptible to immune clearance may provide another valuable treatment option to clinicians. This might prove especially attractive if gene transduction and stable expression occurs before tumors begin downregulating SSTRs.

\section{Central Nervous System}

In chapter 5, I demonstrated proof of principle for Sst-RBS LV transduction in the murine brain and showed that SSTR-2 is expressed in several regions of therapeutic interest (Figures 5-1 and 5-2). Treatment of neurodegenerative diseases has been complicated. Large complexes cannot cross the BBB and most only mask symptoms without addressing disease progression or curative treatment. Promising advancements have been made in response to this need, however, and I will discuss several that could be compatible with Sst-RBS pseudotyped viral vectors.

Neurotrophic factors (NTF) are small (less than $20 \mathrm{kD}$ ) secreted proteins that regulate neuronal development and maintenance. They include nerve growth factor, brain-derived neurotrophic factor, neurotrophin-3 and 4/5, glial cell line-derived factor, and neurturin. NTFs have been shown to slow or even prevent neurological disease by increasing neuronal metabolism and growth and preventing death from nerve injuries and brain traumas. They have been investigated as treatments for multiple conditions involving neuronal destruction such as $A D, H D$ and $P D$, as well as amyotrophic lateral sclerosis (ALS), epilepsy, spinal cord injury, neuropathic pain and stroke. Several clinical trials using AAV2 and LV vectors to transduce various NTFs for AD and PD treatment are underway [135].

RNA interference (RNAi) strategies are another promising avenue for treatment of neurodegenerative diseases and cancer. In vitro and preclinical models have revealed potential therapeutic targets for ALS, AD, HD, PD and frontotemporal dementia among others by selectively targeting mutant genes associated with these conditions. RNAi targets for cancer treatments might include selectively targeted oncogenes or genes that are involved in radio- or chemotherapy resistance mechanisms. As with all other treatment strategies, technical barriers such as specific delivery of therapeutic doses to target cells, long-term expression and the inability to cross the BBB are among the most significant challenges, but AAV and LV vectors are capable of transducing small interfering RNA (siRNA) and short hairpin RNA (shRNA) [136].

Viral vectors to deliver lysosomal enzyme genes such as GUSB have been developed for mouse and dog models [137-139] and successful transduction of GUSB in the murine brain has been documented using several types of viral vectors by both intracranial and intraventricular routes of administration. AAV2 injected into the lateral ventricles of an adult mouse brain had limited affect as it was only capable of accessing the subarachnoid space and transducing pia-arachnoid and hypothalamus cells [104]. Injecting neonatal mice, however, allowed both AAV1 and AAV2 greater access to the developing brain and nearly all brain structures were transduced with expression of GUSB mRNA lasting for up to year in most cases. Limited transduction, mostly choroid plexus and ependymal cells, was seen in AAV5 infected neonatal mice. Neither AAV1 
nor AAV2 transduced any glial or oligodendrocyte cells; but instead, AAV-marker expressing cells strictly colocalized with a neuron-specific marker. Remarkably, direct transduction was not required for protection, as demonstrated by the absence of lesions in non-transduced glial and gray matter cells indicating that GUSB had been secreted by transduced cells and absorbed by neighboring cells [91]. When a VSV G-pseudotyped, HIV-based LV transducing human GUSB was injected into the striatum of adult MPS VII mice, GUSB activity was present at injection sites and stable for up to 16 weeks. Correction of lysosomal storage lesions was observed in areas showing GUSB activity that could be attributed to direct and indirect viral transduction. As in the AAV vector studies, improvement was most pronounced in neurons [90]. The most profound report of lysosomal lesion correction, however, followed injection of a VSV G LV GUSB vector into the lateral ventricles of 6 week old mice. GUSB activity and the presence of lysosomal lesions were assayed after 7 months. The authors documented nearly complete correction of lesions in all brain sections even though GUSB enzyme activity was only significantly detected in one section [140]. These results were presumably due to vector delivery into the CSF which was more capable of effective distribution, as evidenced by RT-PCR detection of vector in each section. However, this may also represent a greater capacity for distribution of secreted enzyme by the CSF originating from transduced cells surrounding the ventricular spaces. In either case, delivery to the CSF and its associated structures could provide an excellent target for Sst-RBS pseudotyped viral vectors.

The choroid plexus (ChP) is an interesting structure located within the ventricular spaces of the brain. It is composed of specialized epithelial cells that are folded into villi surrounding fenestrated capillaries that project out into the ventricles. These frond-like processes filter water and actively transport ions and macromolecules from blood plasma to produce CSF. The ChP is responsible for producing, circulating and filtering toxic substances from the CSF and brain and proteins expressed by the ChP are excreted into the CSF [141, 142]. The ChP forms a continuous structure with the ependymal cell layer. Ependymal cells are of a glial cell lineage. Structurally similar to cuboidal epithelium cells (they are not true epithelial cells since they do not possess a basement membrane or tight junctions), they line the ventricular spaces of the brain and central canal of the spinal cord [143]. Their apical surfaces are covered in cilia and microvilli which circulate and absorb CSF. Since the ependymal layer does not contain tight junctions like the ChP epithelium, CSF and solutes are capable of diffusing through this Brain-CSF interface while the ChP epithelial cells maintain the strict BBB [141]. Ependymal cells may also serve as a source of neuronal stem cells (NSC) after injury [144]. Adult mouse ependymal cells stain positively for all SSTR subtypes, albeit at varying intensities and locations [89]. Additionally, I demonstrated SSTR-2 expression (Figure 5-4) and transduction by Sst-RBS LV (Figure 5-5). Human ChP epithelial cells also stain positively for SSTR-1 and -2 [145]. Thus, the ChP and ependymal cells are good candidates for Sst-RBS LVs transducing lysosomal enzymes and NTFs since they interact directly with CSF which could deliver these secreted proteins throughout the entire brain.

Other targets for CNS delivery are neurogenic regions that contain NSCs capable of giving rise to new neurons and glial cells. If NSCs or another progenitor cell could be transduced this would provide a source of new neurons that now express the target gene. The subventricular zone (SVZ), which is located along the lateral walls of the lateral ventricles, and the subgranular zone (SGZ) of the hippocampal dentate gyrus are the two known neurogenic regions in the adult mammalian brain. The human SVZ is 
composed of four distinct layers: a single layer of ependymal cells lining the ventricle, a gap into which the basal projections of the ependymal cells interact with astrocyte processes, a layer of astrocyte cell bodies, and finally a transitional zone to the brain parenchyma. The SVZ of rodents, dogs and other primates is organized differently in that it lacks the hypocellular gap layer separating the ependymal cells from astrocytes (which can be subdivided into B1 and B2 cells) and contains many more rapidly dividing immature precursor cells (termed $\mathrm{C}$ cells) and migrating neuroblasts (termed A cells) $[94,146,147]$. The SVZ's location and cellular population create a "germinal niche" providing the vasculature and important cellular signals that support neurogenesis [144, 148]. The primary progenitor NSCs of the SVZ have been identified as the B1 population of astrocyte cells [146]. These GFAP positive cells are derived from the same radial glial lineage as ependymal cells $[143,149]$ and remain in close association with them in the SVZ, projecting processes between the ependymal cells and directly contacting the CSF of the lateral ventricles forming a "pinwheel" of ependymal cells surrounding the astrocyte processes $[148,150]$. The B1 cells receive neurogenic signals from the components of the germinal niche stimulating them to develop into the transient amplifying $C$ cells which then become immature neuroblasts [144, 148, 151]. In the rodent SVZ the neuroblasts are supported by the glial sheaths of the B2-type astroctyes and form long chains that migrate along the rostral migratory stream (RMS) to the olfactory bulb where they finally mature [144]. While NSCs have been isolated from the adult human SVZ, the existence of the RMS to olfactory bulb pathway has been a source of debate. Recently, however, it was discovered that migrating neuroblasts are found along the RMS; but as single or double cells, not in long chains as in the rodent RMS [152]. Aside from ependymal cells, the expression of SSTRs on other SVZ cell types has not been documented. If they do express SSTRs this neurogenic region presents an intriguing target cell population. Because they migrate long distances from their location of genesis (and possibly transduction) they have an opportunity to deliver secreted proteins not only at their final destination but along the RMS as well.

The second area of neurogenesis in the adult brain is the SGZ. In this region of the hippocampus, radial glial-like precursors first develop into neuronal progenitor cells and then neuroblasts which migrate throughout the granular layer of the dentate gyrus. To become mature granular cells the new neurons extend dendrites towards the molecular layer and project axons through the hilus into the CA3 region of the hippocampus. The dentate gyrus is strongly positive for SSTR-2 expression (Figure 5-2B) and SSTR-1 and SSTR-2 are also expressed in the CA3 region [153]. As the hippocampus is one of the first regions showing neurodegenerative damage in AD [154], these regions could be excellent candidates for Sst-RBS targeted viral vectors transducing NTFs.

\section{Conclusion}

I demonstrated that a modified MLV glycoprotein, Sst-RBS, has a transduction capacity comparable to that of the WT MLV Env and approaches that of VSV G, the "gold standard" in transduction efficiency in in vitro transduction assays (Figures 3-1 and 4-2). I observed that only one Sst-RBS target receptor subtype, SSTR-5, was capable of reaching this level of transduction, evidently because other receptors tested delivered

virions to degradative environments (Figures 4-9 and 4-10) that were less permissive for entry; whereas SSTR-5 associated virions were directed to more permissive compartments that allowed efficient cytosolic penetration (Figure 4-11). Yet, in vivo transduction assays carried out in the murine brain did not seem to correlate with the in 
vitro data as transduction of both Sst-RBS and VSV G pseudotyped LV vectors was similar (Figures 5-6 and 5-7).

During the course of investigating the transduction capacity of Sst-RBS and its potential for use as an in vitro gene delivery vector, the area of vector diffusion was measured for Sst-RBS and VSV G Envs. Interestingly, Sst-RBS showed a greater potential for vector diffusion than VSV G in which transduction was generally concentrated in a small core area (Figure 5-7). This is a significant observation because delivering therapeutics to brain cells may be more effective if a gene can be delivered to a larger overall area, rather than high transduction in a very small area. For example, genes that encode secreted proteins such as deficient lysosomal enzymes or neurotrophic factors will generate a higher level of therapeutic benefit in this situation because a greater area is covered using fewer vectors. Additionally, since the proteins are secreted, non-transduced cells in the transduction zone also benefit. The coding sequences of the deficient enzymes causing lysosomal storage diseases are less than 5000 base pairs, well within the capacity limits of LV.

SSTR-2 receptor expression was observed in neurons and ependymal cells (Figures 5-3 through 5-5) making these cells types potential targets for gene transduction by Sst-RBS coated viral vectors. Moreover, delivery of the transduction marker mKate2 was demonstrated in ependymal cells. Because the ependymal cells line the ventricles of the brain, they are in close association with the CSF which provides another intriguing delivery route. Since the CSF is naturally distributed throughout the CNS; proteins secreted into the CSF by ependymal cells could potentially gain access all neurological cell types. In the current study, transduction was achieved through direct injection into the murine brain parenchyma. However, this highly invasive method of delivery is not feasible in humans. Less invasive delivery methods exist to access the CSF including spinal taps and intrathecal catheters making delivery to the CSF and its associated structures (the ependymal and choroid plexus cells) an even more attractive option.

One of the more interesting questions remaining after these studies is that of identifying the intracellular compartments to which SSTR-2, -3 and -5 direct the Sst-RBS virus in culture. Using DN-Rab proteins and fluorescent microscopy this information could easily be obtained. Yet, the dramatic difference in transduction capacity between SSTR-2 and SSTR-5 expressing cells did not translate into a similarly low transduction capacity in vivo. This difference may actually represent a cell culture artifact and therefore more useful information would be obtained by concentrating experimental resources on in vivo studies. Moreover, additional information pertaining to in vivo transduction, beyond that generated during this preliminary investigation needs to be attained. First, the sample sizes for transduction and diffusion zone areas should be greater so that large variations due to the nature of individual animals can mitigated. Second, more definitive proof that transduction is SSTR specific should be obtained. Competition assays between Sst-RBS and VSV G pseudotyped vectors in the presence and absence of the inhibitory peptide, SST-14, may be able to answer this question. Third, greater detail regarding the expression of other SSTR subtypes in neurological cell types should be generated. Monoclonal antibodies specific to each of these receptor subtypes can be obtained and immunohistochemical staining protocols similar to those here can be used. In conclusion, demonstrating proof of principle for in vivo gene transduction by the modified retroviral glycoprotein, Sst-RBS, is certainly an exciting advance in the gene therapy field. 


\section{List of References}

1. Albritton, L.M., Tseng, L., Scadden, D., and Cunningham, J.M., A putative murine ecotropic retrovirus receptor gene encodes a multiple membrane-spanning protein and confers susceptibility to virus-infection. Cell, 1989. 57(4): p. 659-666.

2. Albritton, L.M., Kim, J.W., Tseng, L., and Cunningham, J.M., Envelope-binding domain in the cationic amino-acid transporter determines the host range of ecotropic murine retroviruses. J Virol, 1993. 67(4): p. 2091-2096.

3. Kim, J.W., Closs, E.I., Albritton, L.M., and Cunningham, J.M., Transport of cationic amino acids by the mouse ecotropic retrovirus receptor. Nature, 1991. 352(6337): p. 725-8.

4. Lee, S.Y., Zho, Y., and Anderson, W.F., Receptor-mediated moloney murine leukemia virus entry can occur independently of the clathrin-coated-pit-mediated endocytic pathway. J Virol, 1999. 73(7): p. 5994-6005.

5. Lu, X.B. and Silver, J., Ecotropic murine leukemia virus receptor is physically associated with caveolin and membrane rafts. Virol, 2000. 276(2): p. 251-258.

6. Katen, L.J., Januszeski, M.M., Anderson, W.F., Hasenkrug, K.M., and Evans, L.H., Infectious entry by amphotropic as well as ecotropic murine leukemia viruses occurs through an endocytic pathway. J Virol, 2001. 75(11): p. 50185026.

7. Kumar, P., Nachagari, D., Fields, C., Franks, J., and Albritton, L.M., Host cell cathepsins potentiate moloney murine leukemia virus infection. J Virol, 2007. 81(19): p. 10506-10514.

8. Zavorotinskaya, T., Qian, Z.H., Franks, J., and Albritton, L.M., A point mutation in the binding subunit of a retroviral envelope protein arrests virus entry at hemifusion. J Virol, 2004. 78(1): p. 473-481.

9. Roe, T.Y., Reynolds, T.C., Yu, G., and Brown, P.O., Integration of murine leukemia-virus DNA depends on mitosis. EMBO J, 1993. 12(5): p. 2099-2108.

10. Suzuki, Y. and Craigie, R., The road to chromatin - nuclear entry of retroviruses. Nat Rev Microbiol, 2007. 5(3): p. 187-196.

11. Lavillette, D., Russell, S.J., and Cosset, F.L., Retargeting gene delivery using surface-engineered retroviral vector particles. Curr Opin Biotechnol, 2001. 12(5): p. 461-466.

12. Russell, S.J. and Cosset, F.L., Modifying the host range properties of retroviral vectors. J Gene Med, 1999. 1(5): p. 300-311.

13. Verhoeyen, E. and Cosset, F.L., Engineering the surface glycoproteins of lentiviral vectors for targeted gene transfer. Cold Spring Harb Protoc, 2009. 2009(8): p. pdb top59. 
14. Ryu, B.Y., Zavorotinskaya, T., Trentin, B., and Albritton, L.M., The block to membrane fusion differs with the site of ligand insertion in modified retroviral envelope proteins. J Gen Virol, 2008. 89: p. 1049-1058.

15. Zhao, Y., Zhu, L., Lee, S., Li, L., Chang, E., Soong, N.W., Douer, D., and Anderson, W.F., Identification of the block in targeted retroviral-mediated gene transfer. Proc Natl Acad Sci U S A, 1999. 96(7): p. 4005-10.

16. Anderson, J.L. and Hope, T.J., Intracellular trafficking of retroviral vectors: Obstacles and advances. Gene Ther, 2005. 12(23): p. 1667-78.

17. Schneider, U., Bullough, F., Vongpunsawad, S., Russell, S.J., and Cattaneo, R., Recombinant measles viruses efficiently entering cells through targeted receptors. J Virol, 2000. 74(21): p. 9928-36.

18. Bossow, S., Grossardt, C., Temme, A., Leber, M.F., Sawall, S., Rieber, E.P., Cattaneo, R., von Kalle, C., and Ungerechts, G., Armed and targeted measles virus for chemovirotherapy of pancreatic cancer. Cancer Gene Ther, 2011. 18(8): p. 598-608.

19. Frecha, C., Costa, C., Negre, D., Gauthier, E., Russell, S.J., Cosset, F.L., and Verhoeyen, E., Stable transduction of quiescent $t$ cells without induction of cycle progression by a novel lentiviral vector pseudotyped with measles virus glycoproteins. Blood, 2008. 112(13): p. 4843-52.

20. Morizono, K., Xie, Y., Helguera, G., Daniels, T.R., Lane, T.F., Penichet, M.L., and Chen, I.S., A versatile targeting system with lentiviral vectors bearing the biotinadaptor peptide. J Gene Med, 2009. 11(8): p. 655-63.

21. Munch, R.C., Muhlebach, M.D., Schaser, T., Kneissl, S., Jost, C., Pluckthun, A., Cichutek, K., and Buchholz, C.J., Darpins: An efficient targeting domain for lentiviral vectors. Mol Ther, 2011. 19(4): p. 686-93.

22. Morizono, K., Ku, A., Xie, Y., Harui, A., Kung, S.K., Roth, M.D., Lee, B., and Chen, I.S., Redirecting lentiviral vectors pseudotyped with sindbis virus-derived envelope proteins to dc-sign by modification of $n$-linked glycans of envelope proteins. J Virol, 2010. 84(14): p. 6923-34.

23. Sandrin, V., Boson, B., Salmon, P., Gay, W., Negre, D., Le Grand, R., Trono, D., and Cosset, F.L., Lentiviral vectors pseudotyped with a modified rd114 envelope glycoprotein show increased stability in sera and augmented transduction of primary lymphocytes and cd34+ cells derived from human and nonhuman primates. Blood, 2002. 100(3): p. 823-32.

24. Naldini, L., Blomer, U., Gallay, P., Ory, D., Mulligan, R., Gage, F.H., Verma, I.M., and Trono, D., In vivo gene delivery and stable transduction of nondividing cells by a lentiviral vector. Science, 1996. 272(5259): p. 263-7. 
25. Li, F., Ryu, B.Y., Krueger, R.L., Heldt, S.A., and Albritton, L.M., Targeted entry via somatostatin receptors using a novel modified retrovirus glycoprotein that delivers genes at levels comparable to those of wild-type viral glycoproteins. J Virol, 2012. 86(1): p. 373-81.

26. Fass, D., Davey, R.A., Hamson, C.A., Kim, P.S., Cunningham, J.M., and Berger, J.M., Structure of a murine leukemia virus receptor-binding glycoprotein at 2.0 angstrom resolution. Science, 1997. 277(5332): p. 1662-1666.

27. Patel, Y.C., Somatostatin and its receptor family. Front Neuroendocrinol, 1999. 20(3): p. 157-198.

28. Csaba, Z. and Dournaud, P., Cellular biology of somatostatin receptors. Neuropeptides, 2001. 35(1): p. 1-23.

29. Olias, G., Viollet, C., Kusserow, H., Epelbaum, J., and Meyerhof, W., Regulation and function of somatostatin receptors. J Neurochem, 2004. 89(5): p. 1057-1091.

30. Jacobs, S. and Schulz, S., Intracellular trafficking of somatostatin receptors. Mol Cell Endocrinol, 2008. 286(1-2): p. 58-62.

31. Arvigo, M., Gatto, F., Ruscica, M., Ameri, P., Dozio, E., Albertelli, M., Culler, M.D., Motta, M., Minuto, F., Magni, P., and Ferone, D., Somatostatin and dopamine receptor interaction in prostate and lung cancer cell lines. J Endocrinol, 2010. 207(3): p. 309-17.

32. Watt, H., Kharmate, G., and Kumar, U., Somatostatin receptors 1 and 5 heterodimerize with epidermal growth factor receptor: Agonist-dependent modulation of the downstream mapk signalling pathway in breast cancer cells. Cell Signal, 2009. 21(3): p. 428-439.

33. Pfeiffer, M., Koch, T., Schroder, H., Laugsch, M., Hollt, V., and Schulz, S., Heterodimerization of somatostatin and opioid receptors cross-modulates phosphorylation, internalization, and desensitization. J Biol Chem, 2002. 277(22): p. 19762-72.

34. Tulipano, G. and Schulz, S., Novel insights in somatostatin receptor physiology. Eur J Endocrinol, 2007. 156: p. S3-11.

35. Liu, Q.S., Cescato, R., Dewi, D.A., Rivier, J., Reubi, J.C., and Schonbrunn, A., Receptor signaling and endocytosis are differentially regulated by somatostatin analogs. Mol Pharmacol, 2005. 68(1): p. 90-101.

36. Tulipano, G., Stumm, R., Pfeiffer, M., Kreienkamp, H., Höllt, V., and Schulz, S., Differential beta-arrestin trafficking and endosomal sorting of somatostatin receptor subtypes. J Biol Chem, 2004. 279(20): p. 21374-82.

37. Stroh, T., Jackson, A.C., Sarret, P., Dal Farra, C., Vincent, J.P., Kreienkamp, H.J., Mazella, J., and Beaudet, A., Intracellular dynamics of sst(5) receptors in transfected cos-7 cells: Maintenance of cell surface receptors during ligandinduced endocytosis. Endocrinology, 2000. 141(1): p. 354-365. 
38. Roosterman, D., Kempkes, C., Cottrell, G., Padilla, B., Bunnett, N., Turck, C., and Steinhoff, M., Endothelin-converting enzyme-1 degrades internalized somatostatin-14. Endocrinology, 2008. 149(5): p. 2200-7.

39. Roosterman, D., Brune, N.E.I., Kreuzer, O.J., Feld, M., Pauser, S., Zarse, K., Steinhoff, M., and Meyerhof, W., Intracellular degradation of somatostatin-14 following somatostatin-receptor3-mediated endocytosis in rat insulinoma cells. Febs J, 2008. 275(19): p. 4728-4739.

40. Price, J., Turner, D., and Cepko, C., Lineage analysis in the vertebrate nervous system by retrovirus-mediated gene transfer. Proc Natl Acad Sci U S A, 1987. 84(1): p. 156-60.

41. Lois, C., Hong, E.J., Pease, S., Brown, E.J., and Baltimore, D., Germline transmission and tissue-specific expression of transgenes delivered by lentiviral vectors. Science, 2002. 295(5556): p. 868-72.

42. Zavorotinskaya, T. and Albritton, L.M., Suppression of a fusion defect by second site mutations in the ecotropic murine leukemia virus surface protein. J Virol, 1999. 73(6): p. 5034-5042.

43. Kizhatil, K. and Albritton, L.M., Requirements for different components of the host cell cytoskeleton distinguish ecotropic murine leukemia virus entry via endocytosis from entry via surface fusion. J Virol, 1997. 71(10): p. 7145-7156.

44. Day, J.R., Martinez, L.E., Sasik, R., Hitchin, D.L., Dueck, M.E., Richman, D.D., and Guatelli, J.C., A computer-based, image-analysis method to quantify hiv-1 infection in a single-cycle infectious center assay. J Virol Methods, 2006. 137(1): p. 125-133.

45. Gao, G.X. and Goff, S.P., Somatic cell mutants resistant to retrovirus replication: Intracellular blocks during the early stages of infection. Mol Biol Cell, 1999. 10(6): p. $1705-1717$.

46. Zhao, Y., Zhu, L.J., Lee, S., Li, L., Chang, E., Soong, N.W., Douer, D., and Anderson, W.F., Identification of the block in targeted retroviral-mediated gene transfer. Proc Natl Acad Sci U S A, 1999. 96(7): p. 4005-4010.

47. Valsesia-Wittmann, S., Morling, F.J., Nilson, B.H.K., Takeuchi, Y., Russell, S.J., and Cosset, F.L., Improvement of retroviral retargeting by using amino acid spacers between an additional binding domain and the $n$ terminus of moloney murine leukemia virus su. J Virol, 1996. 70(3): p. 2059-2064.

48. Janaka, S.K., Lucas, T.M., and Johnson, M.C., Sequences in gibbon ape leukemia virus envelope that confer sensitivity to hiv-1 accessory protein vpu. J Virol, 2011. 85(22): p. 11945-54.

49. Christodoulopoulos, I. and Cannon, P.M., Sequences in the cytoplasmic tail of the gibbon ape leukemia virus envelope protein that prevent its incorporation into lentivirus vectors. J Virol, 2001. 75(9): p. 4129-38. 
50. Berson, J.F. and Doms, R.W., Structure-function studies of the hiv-1 coreceptors. Semin Immunol, 1998. 10(3): p. 237-48.

51. Battini, J.L., Heard, J.M., and Danos, O., Receptor choice determinants in the envelope glycoproteins of amphotropic, xenotropic, and polytropic murine leukemia viruses. J Virol, 1992. 66(3): p. 1468-75.

52. Albritton, L.M., Tseng, L., Scadden, D., and Cunningham, J.M., A putative murine ecotropic retrovirus receptor gene encodes a multiple membrane-spanning protein and confers susceptibility to virus infection. Cell, 1989. 57(4): p. 659-66.

53. Cosset, F.L., Morling, F.J., Takeuchi, Y., Weiss, R.A., Collins, M.K., and Russell, S.J., Retroviral retargeting by envelopes expressing an $n$-terminal binding domain. J Virol, 1995. 69(10): p. 6314-22.

54. Katane, M., Takao, E., Kubo, Y., Fujita, R., and Amanuma, H., Factors affecting the direct targeting of murine leukemia virus vectors containing peptide ligands in the envelope protein. EMBO Rep, 2002. 3(9): p. 899-904.

55. Mercer, J., Schelhaas, M., and Helenius, A., Virus entry by endocytosis. Annu Rev Biochem, 2010. 79: p. 803-33.

56. Sanders, D.A., Sulfhydryl involvement in fusion mechanisms. Subcell Biochem, 2000. 34: p. 483-514.

57. Colman, P.M. and Lawrence, M.C., The structural biology of type $i$ viral membrane fusion. Nat Rev Mol Cell Biol, 2003. 4(4): p. 309-19.

58. Coffin, J.M., Hughes, S.H., and Varmus, H.E., Retroviruses. 1997, Plainview (NY): Cold Spring Harbor Laboratory Press.

59. Harrison, S.C., Viral membrane fusion. Nat Struct Mol Biol, 2008. 15(7): p. 690698.

60. Goff, S.P., Host factors exploited by retroviruses. Nat Rev Microbiol, 2007. 5(4): p. 253-63.

61. Schelhaas, M., Come in and take your coat off - how host cells provide endocytosis for virus entry. Cellular Microbiology, 2010. 12(10): p. 1378-1388.

62. Huotari, J. and Helenius, A., Endosome maturation. EMBO J, 2011. 30(17): p. 3481-500.

63. Cote, M., Misasi, J., Ren, T., Bruchez, A., Lee, K., Filone, C.M., Hensley, L., Li, Q., Ory, D., Chandran, K., and Cunningham, J., Small molecule inhibitors reveal niemann-pick c1 is essential for ebola virus infection. Nature, 2011. 477(7364): p. 344-8. 
64. Carette, J.E., Raaben, M., Wong, A.C., Herbert, A.S., Obernosterer, G., Mulherkar, N., Kuehne, A.I., Kranzusch, P.J., Griffin, A.M., Ruthel, G., Dal Cin, P., Dye, J.M., Whelan, S.P., Chandran, K., and Brummelkamp, T.R., Ebola virus entry requires the cholesterol transporter niemann-pick c1. Nature, 2011. 477(7364): p. 340-3.

65. Fredericksen, B.L., Wei, B.L., Yao, J., Luo, T.C., and Garcia, J.V., Inhibition of endosomal/lysosomal degradation increases the infectivity of human immunodeficiency virus. J Virol, 2002. 76(22): p. 11440-11446.

66. Wei, B.L., Denton, P.W., O'Neill, E., Luo, T., Foster, J.L., and Garcia, J.V., Inhibition of lysosome and proteasome function enhances human immunodeficiency virus type 1 infection. J Virol, 2005. 79(9): p. 5705-12.

67. Cote, M., Kucharski, T.J., and Liu, S.L., Enzootic nasal tumor virus envelope requires a very acidic ph for fusion activation and infection. J Virol, 2008. 82(18): p. 9023-34.

68. Narayan, S., Barnard, R.J., and Young, J.A., Two retroviral entry pathways distinguished by lipid raft association of the viral receptor and differences in viral infectivity. J Virol, 2003. 77(3): p. 1977-83.

69. Padilla-Parra, S., Marin, M., Kondo, N., and Melikyan, G.B., Synchronized retrovirus fusion in cells expressing alternative receptor isoforms releases the viral core into distinct sub-cellular compartments. PLoS Pathog, 2012. 8(5): p. e1002694.

70. Kadan, M.J., Sturm, S., Anderson, W.F., and Eglitis, M.A., Detection of receptorspecific murine leukemia-virus binding to cells by immunofluorescence analysis. J Virol, 1992. 66(4): p. 2281-2287.

71. Yu, H., Soong, N., and Anderson, W.F., Binding-kinetics of ecotropic (moloney) murine leukemia retrovirus with nih 3t3 cells. J Virol, 1995. 69(10): p. 6557-6562.

72. Zhu, J.Y. and Cunningham, J.M., Minus-strand DNA is present within murine type c ecotropic retroviruses prior to infection. J Virol, 1993. 67(4): p. 2385-2388.

73. Ross, S.R., Schofield, J.J., Farr, C.J., and Bucan, M., Mouse transferrin receptor 1 is the cell entry receptor for mouse mammary tumor virus. Proc Natl Acad Sci U S A, 2002. 99(19): p. 12386-90.

74. Yoshii, H., Kamiyama, H., Minematsu, K., Goto, K., Mizota, T., Oishi, K., Katunuma, N., Yamamoto, N., and Kubo, Y., Cathepsin I is required for ecotropic murine leukemia virus infection in nih3t3 cells. Virol, 2009. 394(2): p. 227-234.

75. Baravalle, G., Schober, D., Huber, M., Bayer, N., Murphy, R.F., and Fuchs, R., Transferrin recycling and dextran transport to lysosomes is differentially affected by bafilomycin, nocodazole, and low temperature. Cell Tissue Res, 2005. 320(1): p. 99-113. 
76. Grinde, B., Effect of carboxylic ionophores on lysosomal protein degradation in rat hepatocytes. Exp Cell Res, 1983. 149(1): p. 27-35.

77. Csaba, Z., Peineau, S., and Dournaud, P., Molecular mechanisms of somatostatin receptor trafficking. J Mol Endocrinol, 2012. 48(1): p. R1-12.

78. Pizzato, M., Blair, E.D., Fling, M., Kopf, J., Tomassetti, A., Weiss, R.A., and Takeuchi, Y., Evidence for nonspecific adsorption of targeted retrovirus vector particles to cells. Gene Ther, 2001. 8(14): p. 1088-1096.

79. Law, S.F., Yasuda, K., Bell, G.I., and Reisine, T., Gi alpha 3 and g(o) alpha selectively associate with the cloned somatostatin receptor subtype sstr2. J Biol Chem, 1993. 268(15): p. 10721-7.

80. Viejo-Borbolla, A., Pizzato, M., Blair, E., and Schulz, T., Insertion of targeting domains into the envelope glycoprotein of moloney murine leukemia virus (momlv)-based vectors modulates the route of mcat-1-mediated viral entry. Virus Res, 2005. 108(1-2): p. 45-55.

81. McClure, M.O., Sommerfelt, M.A., Marsh, M., and Weiss, R.A., The ph independence of mammalian retrovirus infection. J Gen Virol, 1990. 71(4): p. 767-73.

82. Diaz-Salinas, M.A., Romero, P., Espinosa, R., Hoshino, Y., Lopez, S., and Arias, C.F., The spike protein vp4 defines the endocytic pathway used by rotavirus to enter ma104 cells. J Virol, 2013. 87(3): p. 1658-63.

83. Neufeld, E.F. and Muenzer, J., The mucopolysaccharidoses, in The metabolic and molecular bases of inherited disease, Scriver, C.R., Beaudet, A.L., Sly, W.S., and Valle, D., Editors. 2001, McGraw-Hill: New York. p. 3421-3452.

84. Tomatsu, S., Montano, A.M., Dung, V.C., Grubb, J.H., and Sly, W.S., Mutations and polymorphisms in gusb gene in mucopolysaccharidosis vii (sly syndrome). Hum Mutat, 2009. 30(4): p. 511-9.

85. Spencer, B.J. and Verma, I.M., Targeted delivery of proteins across the bloodbrain barrier. Proc Natl Acad Sci U S A, 2007. 104(18): p. 7594-9.

86. Lachmann, R.H., Enzyme replacement therapy for lysosomal storage diseases. Curr Opin Pediatr, 2011. 23(6): p. 588-93.

87. Lachmann, R., Treatments for lysosomal storage disorders. Biochem Soc Trans, 2010. 38(6): p. 1465-8.

88. Fehlmann, D., Langenegger, D., Schuepbach, E., Siehler, S., Feuerbach, D., and Hoyer, D., Distribution and characterisation of somatostatin receptor mrna and binding sites in the brain and periphery. J Physiol Paris, 2000. 94(3-4): p. 265-81.

89. Kumar, U., Colocalization of somatostatin receptor subtypes (sstr1-5) with somatostatin, nadph-diaphorase (nadph-d), and tyrosine hydroxylase in the rat hypothalamus. J Comp Neurol, 2007. 504(2): p. 185-205. 
90. Bosch, A., Perret, E., Desmaris, N., Trono, D., and Heard, J.M., Reversal of pathology in the entire brain of mucopolysaccharidosis type vii mice after lentivirus-mediated gene transfer. Hum Gene Ther, 2000. 11(8): p. 1139-50.

91. Passini, M.A., Watson, D.J., Vite, C.H., Landsburg, D.J., Feigenbaum, A.L., and Wolfe, J.H., Intraventricular brain injection of adeno-associated virus type 1 (aav1) in neonatal mice results in complementary patterns of neuronal transduction to aav2 and total long-term correction of storage lesions in the brains of beta-glucuronidase-deficient mice. J Virol, 2003. 77(12): p. 7034-40.

92. Hannon, J.P., Petrucci, C., Fehlmann, D., Viollet, C., Epelbaum, J., and Hoyer, D., Somatostatin sst2 receptor knock-out mice: Localisation of sst1-5 receptor mrna and binding in mouse brain by semi-quantitative rt-pcr, in situ hybridisation histochemistry and receptor autoradiography. Neuropharmacology, 2002. 42(3): p. 396-413.

93. Kuroda, H., Kutner, R.H., Bazan, N.G., and Reiser, J., A comparative analysis of constitutive and cell-specific promoters in the adult mouse hippocampus using lentivirus vector-mediated gene transfer. J Gene Med, 2008. 10(11): p. 1163-75.

94. Doetsch, F., Garcia-Verdugo, J.M., and Alvarez-Buylla, A., Cellular composition and three-dimensional organization of the subventricular germinal zone in the adult mammalian brain. J Neurosci, 1997. 17(13): p. 5046-61.

95. Rodriguez-Oroz, M.C., Rodriguez, M., Guridi, J., Mewes, K., Chockkman, V., Vitek, J., DeLong, M.R., and Obeso, J.A., The subthalamic nucleus in parkinson's disease: Somatotopic organization and physiological characteristics. Brain, 2001. 124(Pt 9): p. 1777-90.

96. Biffi, A. and Naldini, L., Gene therapy of storage disorders by retroviral and lentiviral vectors. Hum Gene Ther, 2005. 16(10): p. 1133-42.

97. Neufeld, E.F., Lysosomal storage diseases. Annu Rev Biochem, 1991. 60: p. 257-80.

98. Kaplan, A., Achord, D.T., and Sly, W.S., Phosphohexosyl components of a lysosomal enzyme are recognized by pinocytosis receptors on human fibroblasts. Proc Natl Acad Sci U S A, 1977. 74(5): p. 2026-30.

99. Grabowski, G.A., Leslie, N., and Wenstrup, R., Enzyme therapy for gaucher disease: The first 5 years. Blood Rev, 1998. 12(2): p. 115-33.

100. Xu, L., Transduction of hepatocytes after neonatal delivery of a moloney murine leukemia virus based retroviral vector results in long-term expression of $\beta$ glucuronidase in mucopolysaccharidosis vii dogs. Mol Ther, 2002. 5(2): p. 141153.

101. Mango, R., Neonatal retroviral vector-mediated hepatic gene therapy reduces bone, joint, and cartilage disease in mucopolysaccharidosis vii mice and dogs. Mol Genet Metab, 2004. 82(1): p. 4-19. 
102. Macsai, C.E., Derrick-Roberts, A.L.K., Ding, X., Zarrinkalam, K.H., Mclntyre, C., Anderson, P.H., Anson, D.S., and Byers, S., Skeletal response to lentiviral mediated gene therapy in a mouse model of mps vii. Mol Genet Metab, 2012. 106(2): p. 202-213.

103. Ponder, K.P., Melniczek, J.R., Xu, L., Weil, M.A., O'Malley, T.M., O'Donnell, P.A., Knox, V.W., Aguirre, G.D., Mazrier, H., Ellinwood, N.M., Sleeper, M., Maguire, A.M., Volk, S.W., Mango, R.L., Zweigle, J., Wolfe, J.H., and Haskins, M.E., Therapeutic neonatal hepatic gene therapy in mucopolysaccharidosis vii dogs. Proc Natl Acad Sci U S A, 2002. 99(20): p. 13102-7.

104. Passini, M.A. and Wolfe, J.H., Widespread gene delivery and structure-specific patterns of expression in the brain after intraventricular injections of neonatal mice with an adeno-associated virus vector. J Virol, 2001. 75(24): p. 12382-92.

105. Blomer, U., Naldini, L., Kafri, T., Trono, D., Verma, I.M., and Gage, F.H., Highly efficient and sustained gene transfer in adult neurons with a lentivirus vector. $\mathrm{J}$ Virol, 1997. 71(9): p. 6641-9.

106. Nixon, R.A. and Cataldo, A.M., The endosomal-lysosomal system of neurons: New roles. Trends neurosci, 1995. 18(11): p. 489-496.

107. Von Bartheld, C.S. and Altick, A.L., Multivesicular bodies in neurons: Distribution, protein content, and trafficking functions. Prog Neurobiol, 2011. 93(3): p. 313340.

108. Tai, H.C. and Schuman, E.M., Ubiquitin, the proteasome and protein degradation in neuronal function and dysfunction. Nat Rev Neurosci, 2008. 9(11): p. 826-38.

109. Csaba, Z., Lelouvier, B., Viollet, C., El Ghouzzi, V., Toyama, K., Videau, C., Bernard, V., and Dournaud, P., Activated somatostatin type 2 receptors traffic in vivo in central neurons from dendrites to the trans golgi before recycling. Traffic, 2007. 8(7): p. 820-34.

110. Mizuno-Yamasaki, E., Rivera-Molina, F., and Novick, P., Gtpase networks in membrane traffic. Annu Rev Biochem, 2012. 81: p. 637-59.

111. Grosshans, B.L., Ortiz, D., and Novick, P., Rabs and their effectors: Achieving specificity in membrane traffic. Proc Natl Acad Sci U S A, 2006. 103(32): p. 11821-7.

112. Hutagalung, A.H. and Novick, P.J., Role of rab gtpases in membrane traffic and cell physiology. Physiol Rev, 2011. 91(1): p. 119-49.

113. Takahashi, S., Kubo, K., Waguri, S., Yabashi, A., Shin, H.W., Katoh, Y., and Nakayama, K., Rab11 regulates exocytosis of recycling vesicles at the plasma membrane. J Cell Sci, 2012. 125(Pt 17): p. 4049-57. 
114. Mire, C.E., White, J.M., and Whitt, M.A., A spatio-temporal analysis of matrix protein and nucleocapsid trafficking during vesicular stomatitis virus uncoating. PLoS Pathog, 2010. 6(7): p. e1000994.

115. Sieczkarski, S.B. and Whittaker, G.R., Differential requirements of rab5 and rab7 for endocytosis of influenza and other enveloped viruses. Traffic, 2003. 4(5): $p$. 333-43.

116. Mercer, J. and Helenius, A., Virus entry by macropinocytosis. Nat Cell Biol, 2009. 11(5): p. 510-20.

117. Saeed, M.F., Kolokoltsov, A.A., Albrecht, T., and Davey, R.A., Cellular entry of ebola virus involves uptake by a macropinocytosis-like mechanism and subsequent trafficking through early and late endosomes. PLoS Pathog, 2010. 6(9): p. e1001110.

118. Nanbo, A., Imai, M., Watanabe, S., Noda, T., Takahashi, K., Neumann, G., Halfmann, P., and Kawaoka, Y., Ebolavirus is internalized into host cells via macropinocytosis in a viral glycoprotein-dependent manner. PLoS Pathog, 2010. 6(9): p. e1001121.

119. Zhu, P., Chertova, E., Bess, J., Jr., Lifson, J.D., Arthur, L.O., Liu, J., Taylor, K.A., and Roux, K.H., Electron tomography analysis of envelope glycoprotein trimers on hiv and simian immunodeficiency virus virions. Proc Natl Acad Sci U S A, 2003. 100(26): p. 15812-7.

120. Bachrach, E., Dreja, H., Lin, Y.L., Mettling, C., Pinet, V., Corbeau, P., and Piechaczyk, M., Effects of virion surface gp120 density on infection by hiv-1 and viral production by infected cells. Virol, 2005. 332(1): p. 418-29.

121. Zheng, Y.H., Jeang, K.T., and Tokunaga, K., Host restriction factors in retroviral infection: Promises in virus-host interaction. Retrovirology, 2012. 9: p. 112.

122. Goff, S.P., Retrovirus restriction factors. Mol Cell, 2004. 16(6): p. 849-59.

123. Stremlau, M., Perron, M., Lee, M., Li, Y., Song, B., Javanbakht, H., Diaz-Griffero, F., Anderson, D.J., Sundquist, W.I., and Sodroski, J., Specific recognition and accelerated uncoating of retroviral capsids by the trim5alpha restriction factor. Proc Natl Acad Sci U S A, 2006. 103(14): p. 5514-9.

124. Lukic, Z., Hausmann, S., Sebastian, S., Rucci, J., Sastri, J., Robia, S.L., Luban, J., and Campbell, E.M., Trim5alpha associates with proteasomal subunits in cells while in complex with hiv-1 virions. Retrovirology, 2011. 8: p. 93.

125. Danielson, C.M., Cianci, G.C., and Hope, T.J., Recruitment and dynamics of proteasome association with rhtrim5alpha cytoplasmic complexes during hiv-1 infection. Traffic, 2012. 13(9): p. 1206-17.

126. Grutter, M.G. and Luban, J., Trim5 structure, hiv-1 capsid recognition, and innate immune signaling. Curr Opin Virol, 2012. 2(2): p. 142-50. 
127. Rein, A., Murine leukemia viruses: Objects and organisms. Adv Virol, 2011. 2011: p. 403419.

128. Frilling, A., Akerstrom, G., Falconi, M., Pavel, M., Ramos, J., Kidd, M., and Modlin, I.M., Neuroendocrine tumor disease: An evolving landscape. Endocr Relat Cancer, 2012. 19(5): p. R163-85.

129. Watt, H., Kharmate, G., and Kumar, U., Biology of somatostatin in breast cancer. Mol Cell Endocrinol, 2008. 286(1-2): p. 251-261.

130. Reubi, J.C. and Laissue, J.A., Multiple actions of somatostatin in neoplastic disease. Trends Pharmacol Sci, 1995. 16(3): p. 110-5.

131. Pyronnet, S., Bousquet, C., Najib, S., Azar, R., Laklai, H., and Susini, C., Antitumor effects of somatostatin. Mol Cell Endocrinol, 2008. 286(1-2): p. 230237.

132. Sun, L.C. and Coy, D.H., Somatostatin receptor-targeted anti-cancer therapy. Curr Drug Deliv, 2011. 8(1): p. 2-10.

133. Reubi, J., Waser, B., Cescato, R., Gloor, B., Stettler, C., and Christ, E., Internalized somatostatin receptor subtype 2 in neuroendocrine tumors of octreotide-treated patients. J Clin Endocrinol Metab, 2010. 95(5): p. 2343-50.

134. Mitra, P., De, A., Ethier, M.F., Mimori, K., Kodys, K., Shibuta, K., Mori, M., Madison, J.M., Miller-Graziano, C., and Barnard, G.F., Loss of chemokine sdf1alpha-mediated cxcr4 signalling and receptor internalization in human hepatoma cell line hepg2. Cell Signal, 2001. 13(5): p. 311-9.

135. Lim, S.T., Airavaara, M., and Harvey, B.K., Viral vectors for neurotrophic factor delivery: A gene therapy approach for neurodegenerative diseases of the cns. Pharmacol Res, 2010. 61(1): p. 14-26.

136. Seyhan, A.A., Rnai: A potential new class of therapeutic for human genetic disease. Hum Genet, 2011. 130(5): p. 583-605.

137. Ray, J., Bouvet, A., DeSanto, C., Fyfe, J.C., Xu, D., Wolfe, J.H., Aguirre, G.D., Patterson, D.F., Haskins, M.E., and Henthorn, P.S., Cloning of the canine betaglucuronidase cdna, mutation identification in canine mps vii, and retroviral vector-mediated correction of mps vii cells. Genomics, 1998. 48(2): p. 248-53.

138. Gao, C., Delivery of a retroviral vector expressing human $\beta$-glucuronidase to the liver and spleen decreases lysosomal storage in mucopolysaccharidosis vii mice. Mol Ther, 2000. 2(3): p. 233-244.

139. Smith, L.J., Martin, J.T., O'Donnell, P., Wang, P., Elliott, D.M., Haskins, M.E., and Ponder, K.P., Effect of neonatal gene therapy on lumbar spine disease in mucopolysaccharidosis vii dogs. Mol Genet Metab, 2012. 
140. Bielicki, J., McIntyre, C., and Anson, D.S., Comparison of ventricular and intravenous lentiviral-mediated gene therapy for murine mps vii. Mol Genet Metab, 2010. 101(4): p. 370-82.

141. Johanson, C.E., Duncan, J.A., 3rd, Klinge, P.M., Brinker, T., Stopa, E.G., and Silverberg, G.D., Multiplicity of cerebrospinal fluid functions: New challenges in health and disease. Cerebrospinal Fluid Res, 2008. 5: p. 10.

142. Falcao, A.M., Marques, F., Novais, A., Sousa, N., Palha, J.A., and Sousa, J.C., The path from the choroid plexus to the subventricular zone: Go with the flow! Front Cell Neurosci, 2012. 6: p. 34.

143. Spassky, N., Adult ependymal cells are postmitotic and are derived from radial glial cells during embryogenesis. J Neurosci, 2005. 25(1): p. 10-18.

144. Ming, G.L. and Song, H., Adult neurogenesis in the mammalian brain: Significant answers and significant questions. Neuron, 2011. 70(4): p. 687-702.

145. Katz, S.E., Klisovic, D.D., O'Dorisio, M.S., Lynch, R., and Lubow, M., Expression of somatostatin receptors 1 and 2 in human choroid plexus and arachnoid granulations: Implications for idiopathic intracranial hypertension. Arch Ophthalmol, 2002. 120(11): p. 1540-3.

146. Doetsch, F., Caille, I., Lim, D.A., Garcia-Verdugo, J.M., and Alvarez-Buylla, A., Subventricular zone astrocytes are neural stem cells in the adult mammalian brain. Cell, 1999. 97(6): p. 703-16.

147. Quiñones-Hinojosa, A. and Chaichana, K., The human subventricular zone: A source of new cells and a potential source of brain tumors. Exp Neurol, 2007. 205(2): p. 313-324.

148. Ihrie, R.A. and Alvarez-Buylla, A., Lake-front property: A unique germinal niche by the lateral ventricles of the adult brain. Neuron, 2011. 70(4): p. 674-86.

149. Ihrie, R.A. and Alvarez-Buylla, A., Cells in the astroglial lineage are neural stem cells. Cell Tissue Res, 2008. 331(1): p. 179-91.

150. Mirzadeh, Z., Merkle, F.T., Soriano-Navarro, M., Garcia-Verdugo, J.M., and Alvarez-Buylla, A., Neural stem cells confer unique pinwheel architecture to the ventricular surface in neurogenic regions of the adult brain. Cell Stem Cell, 2008. 3(3): p. 265-78.

151. Alvarez-Buylla, A. and Lim, D.A., For the long run: Maintaining germinal niches in the adult brain. Neuron, 2004. 41(5): p. 683-6.

152. Wang, C., Liu, F., Liu, Y.Y., Zhao, C.H., You, Y., Wang, L., Zhang, J., Wei, B., Ma, T., Zhang, Q., Zhang, Y., Chen, R., Song, H., and Yang, Z., Identification and characterization of neuroblasts in the subventricular zone and rostral migratory stream of the adult human brain. Cell Res, 2011. 21(11): p. 1534-50. 
153. Bando, S.Y., Alegro, M.C., Amaro, E., Jr., Silva, A.V., Castro, L.H., Wen, H.T., Lima Lde, A., Brentani, H., and Moreira-Filho, C.A., Hippocampal ca3 transcriptome signature correlates with initial precipitating injury in refractory mesial temporal lobe epilepsy. PLoS One, 2011. 6(10): p. e26268.

154. Thompson, P.M. and Vinters, H.V., Pathologic lesions in neurodegenerative diseases. Prog Mol Biol Transl Sci, 2012. 107: p. 1-40. 


\section{Vita}

Robin Krueger was born in 1976 in Jackson, Michigan. She graduated with her Bachelor in Science from Jacksonville University in 1998 with a major in Biology and a minor in Chemistry. After receiving a Masters in Public Health (MPH) in 2001 from The University of South Florida she worked for the Florida Department of Public Health, Virology Division then as a research assistant for The University of South Florida Center for Biological Defense. In 2007 she entered the Integrated Program in Biomedical Sciences (IPBS) Microbial Pathogenesis, Immunology, and Inflammation track at The University of Tennessee Health Science Center. She then joined Dr. Lorraine Albritton's laboratory studying the murine leukemia virus envelope protein and entry of a modified entry targeted glycoprotein. She graduated with the degree Doctor of Philosophy in 2013 with a focus on molecular biology and virology. 